\title{
A DISTRIBUIÇÃO EXPONENCIAL APLICADA A TESTES DE VIDA
}

JOSEFA ALVAREZ ALVAREZ

Fisica

Orientador: Prof. Dr. IZAIAS RANGEL NOGUEIRA

\begin{abstract}
Dissertaçāo apresentada à Escola Superior de Agricultura "Luiz de Queiroz", da Universidade de São Paulo. para obtençāo do título de Mestre em Estatistica e Experimen. taçāo Agronômica.
\end{abstract}

P I R A C I C A B A

Estado de São Paulo - Brasil

Dezembro, 1981 


\author{
Aos meus irmãos, Lorenzo e \\ Marcos pela constante \\ ded icação,
}

Ao Prof. Victor, pela amizade

e incentivo.

A meu pai, in memorian,

à minha mãe,

D E D I C O. 
AGRADEC IMENTOS

Ao Professor Dr. Izaias Rangel Nogueira, Professor TituIar do Departamento de Matemática e Estatística da Escola Superior de Agri cultura "Luiz de Queiroz" pela orientação segura, o qual, mais do que or $\underline{\text { I }}$ entador, foi amigo.

Ao Professor Catedrātico Dr.Frederico Pimentel Gomes, Che fe do Departamento de Matemática e Estatística da "ESALQ", pelo seu valor humano e amizade.

Ao Professor Victor Mirshawka, pelos ensinamentos recebidos, constantes incentivos e ajudas prestadas.

Ao Dr. Humberto de Campos, Coordenador do Curso de PósGraduação de Estatística e Experimentação Agronômica da "ESALQ", pela ami zade.

Aos demais Professores do Departamento de Matemātica e Es tatística da Escola Superior de Agricultura "Luiz de Queiroz" pelos ensinamentos recebidos e proveitosos diālogos.

Aos amigos da Fundação Armando Âlvares Penteado, da Universidade Mackenzíe, e da Faculdade de Engenharia São Paulo em especial, aos colegas das disciplinas de Estatística-Pesquisa Operacional e Cálculo Numērico-Ciência da Computação.

A Fundação Armando Âlvares Penteado, que tornou possível a realização do Curso de Pōs-Graduação. 
Ao Sr. Toshiro Iqueda e a Srta. Mara De Salvo pela execução dos trabalhos datilogrâficos e, principalmente pela amizade.

Ās Srtas. Maria Izalina Ferreira Alves e Rosa Maria A1ves, pelo companheirismo e amizade.

Aos colegas do Curso de Pós-Graduação pelo excelente convívio durante o curso.

Aos amigos da Livraria Nobel.

A todos que, de uma forma ou de outra, contribuiram para a realização desse trabalho. 
INDICE

Päg.

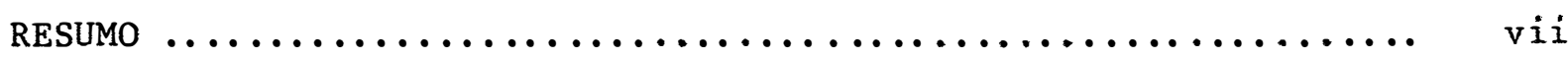

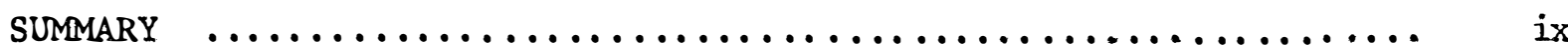

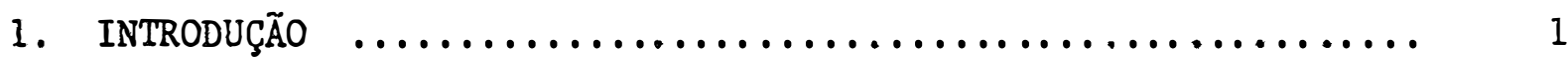

2. REVISÃo de Literatura .......................... 4

2.1 - Estimação para a Distribuição Exponencial Monoparamētri-

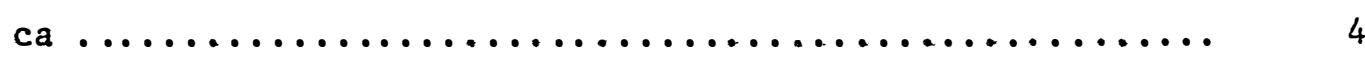

2.2 - Estimação para a Distribuição Exponencial Biparamêtrica. 8

2.3 - Testes de Hipöteses ......................... 10

2.3.1 - Distribuição Exponencial Monoparamêtrica ....... 10

2.3.2 - Distribuição Exponencial Biparamētrica ........ 11

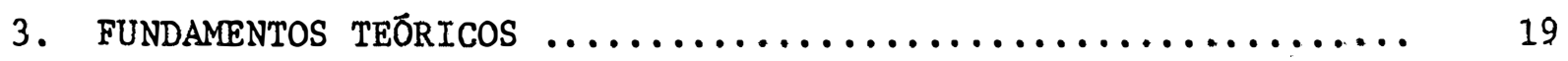

3.1 - Propriedades Estatỉsticas da Distribuição Exponencial .. 19

3.2 - Estimação para a Distribuição Exponencial Monoparamētrí

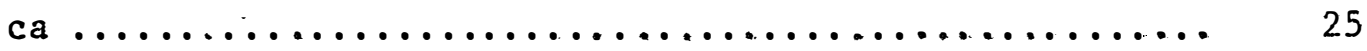

3.2.1 - Censura Tipo II. Amostragem sem reposição ..... 25

3.2.2 - Censura Tipo I. Amostragem com reposição ..... 31

3.2 .3 - Censura Tipo I. Amostragem sem reposição ...... 33

3.2 .4 - Censura Tipo I. Amostragem com reposiçäo ...... 37

3.3 - Estimação da Função de Confiabilidade para a Distribuição Exponencial Monoparamētrica ................. 41

3.4 - Estimação para a Distribuição Exponencial Biparamêtrica. $\quad 43$ 
3.5 - Testes de Hipôteses ......................... 49

3.5.1.-: Censura Tipo II ........................ 53

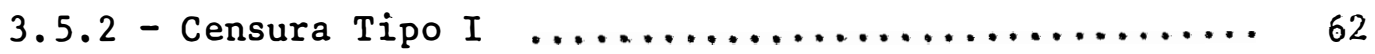

3.5 .3 - Tamanho da Amostra ........................... 67

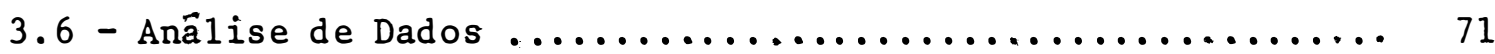

3.6 .1 - Teste qui-quadrado $\ldots \ldots \ldots \ldots \ldots \ldots \ldots \ldots \ldots \ldots \ldots$

3.6.2 - Teste para verificar se a taxa de falhas permanece

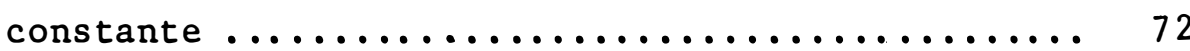

3.6 .3 - Técnicas Gräficas ........................ 73

4. EXEMPLOS NUMERICOS $\ldots \ldots \ldots \ldots \ldots \ldots \ldots \ldots \ldots \ldots \ldots \ldots \ldots \ldots \ldots$

4.1 - Distribuição Exponencial Monoparamētrica ............ 76

4.1 .1 - Censura Tipo II. Amostragem sem reposição ...... 76

4.1 .2 - Censura Tipo II. Amostragem com reposição ...... 79

4.1 .3 - Censura Tipo I. Amostragem com reposição ....... 81

4.1 .4 - Censura Tipo I. Amostragem sem reposição ....... 82

4.2 - Distribuição Exponencial Biparamētrica ............ 83

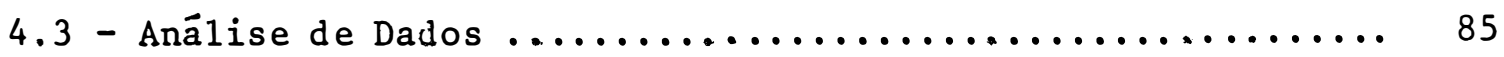

4.3 .1 - Teste qui-quadrado ..................... 85

4.3.2 - Teste para verificar se a taxa de falhas permanece

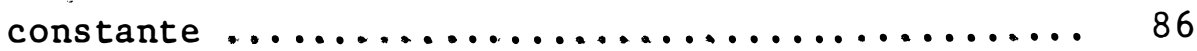

4.3 .3 - Técnica Gräfica .................... 87

5. CONCLUSÕES $\ldots \ldots \ldots \ldots \ldots \ldots \ldots \ldots \ldots \ldots \ldots \ldots \ldots \ldots \ldots \ldots \ldots$

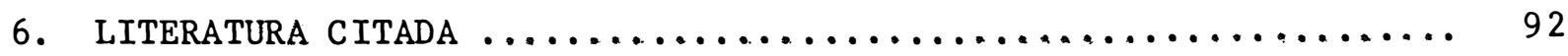

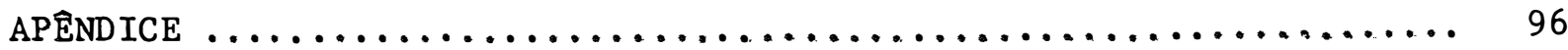


Candidata: JOSEFA ALVAREZ ALVAREZ

Orientador: Dr. Izaias Rangel Nogueira

R E S Ù M O

0 presente trabalho teve como objetivo fornecer uma anãli se mais cuidadosa da Inferência Estatística, quando se utiliza a distribuição exponencial como um modelo para falhas.

Tal distribuiçāo é bastante útil na ārea da confiabilidade e testes de vida. E um conceito relevante que surge neste âmbito, $\widehat{e}$ o de Amostra Censurada.

Foram considerados para a distribuição exponencial monoparamétrica quatro casos: censura tipo II, amostragem sem reposiçāo e com reposição; censura tipo I, amostragem sem reposição e com reposição. Para cada situação, determinaram-se os estimadores e os respectivos intervalos de confiança para os parâmetros, e a confiabilidade.

Fez-se tambēm a anâlise da distribuição exponencial biparamētrica, encontrando-se os estimadores para os parâmetros, vida média e vida mỉnima, bem como os intervalos de confiança. 
viii.

Quanto aos testes de hipótese, foram eles desenvolvidos, supondo-se que a distribuiçăo ê exponencial e que houve censura tipo I ou tipo II, encontrando-se para cada situação a estati̊stica teste e as regiôes crïticas.

Alêm disso, têcnicas gräficas e analiticas foram aplicadas às amostras para verificar-se a validade do modelo exponencial. 
Candidate: JOSEFA ALVAREZ ALVAREZ

Adviser: Dr. Izaias Rangel Nogueira

This paper had the objective to furnish a more careful Inference Statistic, when we use the exponential distribution as a model for failure.

Such distribution is very practical in the reliability area and life tests and a relevant concept that arises in this circuit, is the one of Censured Samples.

Four cases were considered for one-parameter exponential distribution: type II censure, sampling without and with replacement, type I censure, sampling without and with replacement. For each situation, the estimators was determined and the respective confidence intervals for the parameter and the reliability.

We also made the bi-parameter exponential distribution analysis, finding the estimators for the parameters mean and minimum life, as well as the confidence intervals. 
As to the life statistic tests these were developed, suppo sing that the distribution is exponential and there was type I or type II censure, finding for each situation a statistic test and critic regions. Despite of this, graphif and analystic technics were applied to the samples to verify the validity of the exponential model. 
1. INTRODUÇÃO

Um teste de vida para um equipamento é o processo de colocar uma unidade sobre um específico conjunto de testes, medindo-se o tempo até que este falhe. Quanto a estas falhas, podem ser elas classificadas em três modalidades: as de mortalidade infantil, as aleatōrias e as devido ao desgaste.

As falhas de mortalidade precoce são devidas à deficiência do projeto, da fabricação e da inspeção.

A classe de falhas aleatōrias é devida ao esforço repenti no,condição extrema e assim por diante. A outra classe de falhas representa as falhas por desgaste, isto é devido à deterioração do equipamento. A distribuição exponencial é utilizada durante o período em que as falhas ocorrem aleatoriamente. Durante este período não existe um mecanismo de falha predominantemente responsável.

Admite-se que pode haver mais de uma causa contribuindo 
para uma falha particular e que em certos casos pode não haver uma distinção totalmente clara entre algumas das causas. De modo que, em grupo de unidades aparentemente iguais por exemplo, se as lâmpadas incandescentes forem colocadas em teste, os instantes em que ocorrem as falhas irão variar.

Pode-se descobrir as causas que contribuem para essa variação. Assim è possĩvel, talvez, que algumas dessas lâmpadas teninam se encontrado em um ambiente onde havia determinada vibração. Então é provāvel que essas lâmpadas tenham vida menor que aquelas que não sofreram vibração. Everdade tambëm que, se nenhuma causa assinalāvel for encontrada, ainda assim as lâmpadas terão tempos de vida distintos. En qualquer caso, o tempo de vida das lâmpadas será uma variável aleatória.

Obviamente existirā grande vantagem em se determinar a fun ção de densidade que descreve precisamente o comportamento da variävel aleatöria; contudo, isto poderâ ser bem mais difícil, quando existem uma ou mais, causas assinaláveis de variação.

Mas è natural que, se os tempos de vida de alguns componentes, sob um conjunto dado de condições,forem adequadamente descritos por uma distribuição de probabilidades, então um benefício considerável será obtido.

Para tanto, dados devem ser utilizados a fim de se estima rem os parâmetros de uma determinada distribuição de probabilidades e suas caracteristicas. Essas estimativas podem ser utilizadas para fazer predições, determinar configurações dos componentes no sistema, determinar um procedimento para reposição, especificar perïodos de garantia e assim por diante. 
Porém, a natureza dos dados observados em testes de vida são freqüentemente diferentes dos dados estatîsticos usuais.

Assim, se estamos interessados na vida ütil de um certo tipo de componente, colocam-se $\underline{n}$ unidades em teste e marcam-se os tempos de falhas, de modo que resulte uma amostra ordenada.

Devido a 1 imitações de tempo ou a limitações econômicas, o experimento pode terminar antes que todas as unidades tenham falhado; diz-se, então, que a amostragem foi censurada.

Portanto, uma amostragem pode ser censurada para um parti cular tempo, ou quando um nümero particular de falhas ocorrem. Se o experimento termina para um determinado tempo representado por $\underline{t}^{*}$, antes que todas as $\underline{n}$ unidades tenham falhado, diz-se que se realizou uma amostragem com censura tipo I. Jâ a amostragem com censura tipo II ocorre quando o experimento termina para um particular nümero de falhas ou seja apỏs a $\underline{r}$-ësima falha.

No primeiro caso, o nủmero de falhas è uma variável aleatöria; no segundo caso, a duração do experimento è uma variâvel aleatôria.

Neste trabalho serä apresentada uma metodologia que pode ser aplicada a dados resultantes de testes de vida, quando a distribuição é exponencial. Mostram-se as técnicas de Estimação e os Testes de Hipōtese relativos aos parâmetros desta distribuição. 


\section{REVISÃO DE LITERATURA}

2.1 - Estimação para a Distribuiçāo Exponencial Monoparamétrica

Inicialmente discute-se os problemas de estimação quando o tempo de vida, T, pode ser descrito por

$$
f(t ; \theta)=\frac{1}{\theta} \exp \left(-\frac{t}{\theta}\right) ; \quad I_{[0, \infty)}(t) ; \quad \theta>0
$$

São de uso comum duas classes de estimação denominadas es timação pontual e por intervalo.

No caso da estimação pontual, considera-se o mêtodo da mâ xima verossimilhança. Sua forma geral, para propōsitos da Estatỉstica foi proposta por FISHER (1922) numa conceituação considerada de máxima importância no desenvolvimento da teoria Estatîstica moderna.

A teoria moderna e a terminologia de intervalos de confiança for am desenvolvidas por NEYMAN (1935).

Baseando-se no trabalho de JACOBSON (1947), EPSTEIN e SOBEL (1953) analisaram o caso da distribuição exponencial e obtiveram o estimador de máxima verossimilhança para a vida mêdia: 


$$
\hat{\theta}=\frac{\sum_{i=1}^{r} t_{i, n}+(n-r) t_{r, n}}{r} ; r>0,
$$

onde os primeiros tempos de falhas observados em uma amostra de tamanho $\underline{\mathrm{n}}$ são: $t_{1, n} \leqslant t_{2, n} \leqslant \cdots \leqslant t_{r, n}$. Mostraram tambēm que a distribuição de $\hat{\theta}$ é qui quadrado e que depende de $\underline{\mathbf{r}}$ e não de $\underline{\mathrm{n}}$. O estimador de $\underline{\theta}$ é imparcial.

EPSTEIN (1960) discute quatro processos de estimação pontual e por intervalo para dados de teste de vida. Os dois primeiros processos podem ser aplicados, quando a distribuição é exponencial; o tercei ro e quarto processos são não paramétricos.

Caso I: 0 estimador do parâmetro $\hat{\theta} \bar{e}$ dado por:

$$
\hat{\theta}=\frac{T r, n}{r} ; r>0
$$

se o experimento termina apōs a $\underline{r}$-ésima falha e a amostragem è sem reposição, $T_{r, n}$ é dado por:

$$
T_{r, n}=\sum_{i=1}^{r} t_{i, n}+(n-r) t_{r, n},
$$

quando a amostragem é com reposição, $T_{r, n} \bar{e}$ dado por:

$$
\mathrm{T}_{\mathrm{r}, \mathrm{n}}=\mathrm{nt}_{\mathrm{r}, \mathrm{n}}
$$

Como $\frac{2 r \hat{\theta}}{\theta}=\frac{2 T}{r} \frac{r}{\theta}, n$ possui distribuição de qui-quadrado com $\underline{2 r}$ graus de liberdade, pode-se obter o intervalo de confiança para o parâmetro $\underline{\theta}$ que é dado por: 


$$
\frac{2 \mathrm{~T}_{\mathrm{r}, \mathrm{n}}}{x_{2 \mathrm{r}, \alpha / 2}^{2}} \leqslant \theta \leqslant \frac{2 \mathrm{~T}_{\mathrm{r}, \mathrm{n}}}{x_{2 \mathrm{r}, 1-\alpha / 2}^{2}}
$$

Caso II: Quando a duração do experimento é fixada, as uni dades que falham podem ou não ser repostas. 0 estimador da vida mêdia, quando as unidades são repostas, é dado por:

$$
\hat{\theta}=\frac{T}{r} ; \quad r>0,
$$

onde $\mathrm{T}=\mathrm{nt}{ }^{*} ; \underline{t}^{*} \overline{\mathrm{e}}$ o tempo de duração do experimento. 0 intervalo de con fiança é:

$$
-\frac{2 T}{x_{2 r+2, \alpha / 2}^{2}} \leqslant \theta \leqslant \frac{2 T}{x_{2 r, 1-\alpha / 2}^{2}}
$$

Caso III: Se n unidades são colocadas em um teste de duração $t^{*}$, e as que falharem não forem repostas, pode-se representar por $\mathrm{X}$ ○ nümero de unidades que falham durante o teste e ter-se-â, então a probabilidade de obter $\underline{r}$ falhas:

$$
P(X=r)=\left({ }_{r}^{n}\right) p^{r} q^{n-r} ; \quad I_{\{0,1,2, \ldots, n\}}(r), q=1-p \quad .
$$

Da relação da distribuição binomial com a distribuição $F$ tem-se:

$$
P(X \leqslant x)=P\left(F_{v_{1}, \nu_{2}}, \alpha \leqslant \frac{n-r}{r+1} \frac{p}{1-p}\right)
$$

onde $v_{1}=2 r+2$ e $v_{2}=2 n-2 r$. Neste caso, pode-se fazer a seguinte afir mação não paramêtrica com uma confiança $\gamma(0<\gamma<1)$ de que ao menos $\underline{q}$ da população sobrevive à duração de tempo $\underline{t}^{*}$. o valor de $q \underline{e}$ dado por: 


$$
q=\left[1+\left(\frac{r+1}{n-r}\right) v_{1}, v_{2}, \alpha\right]^{-1},
$$

sendo $v_{1}=2 r+2$ e $v_{2}=2 n-2 r$.

Em particular, quando a distribuição é exponencial, o limite inferior do parâmetro $\underline{\theta}$ obtido de $q=\exp \left(\frac{-t^{*}}{\theta}\right)$, assim tem-se:

$$
\hat{\theta}_{I}>t^{*}\left\{\ln \left[1+\left(\frac{r+1}{n-r}\right) F_{\nu_{1}, \nu_{2}, \alpha}\right]\right\}^{-1} .
$$

Caso IV: As unidades são retiradas aleatoriamente de alguma população e testadas, uma por vez, durante o tempo $\underline{t}^{*}$. Entende-se que ocorreu uma falha se a unidade parar defuncionar antes de $t^{*}$. 0 teste continua até que um determinado nümero de falhas $\underline{x}$ ocorra. Suponha-se que a $\underline{r}$-ésima falha ề obtida, quando a ñ-êsima unidade è colocada em teste, tal que

$$
P(X=r)=\left(\begin{array}{c}
n-1 \\
r-1
\end{array}\right) p^{r} q^{n-r} ; \quad I_{\{r, r+1, r+2, \ldots\}}(n), \quad q=1-p .
$$

Neste caso, pode-se afirmar com uma confiança $\gamma(0<\gamma<1)$ que ao menos uma fração q da população sobrevive à duração de tempo $\underline{t}^{*}$, onde $\underline{q}$ é dado por:

$$
q=\left[1+\left(\frac{x}{n-x}\right) F_{\nu_{1}, \nu_{2}, \alpha}\right]^{-1},
$$

sendo $\quad v_{1}=2 \mathrm{r}$ e $v_{2}=2 \mathrm{n}-2 \mathrm{r}$.

Quando a distribuição é exponencial, o limite inferior de $\underline{\theta}$ è obtido de $q=\exp \left(\frac{-t^{*}}{\theta}\right)$, assim tem-se:

$$
\hat{\theta}_{I}>t^{*}\left(\ln \left[1+\left(\frac{r}{n-r}\right) F_{v_{1}}, v_{2}, \alpha\right]\right)^{-1},
$$

sendo $v_{1}=2 \mathrm{r}$ e $v_{2}=2 \mathrm{n}-2 \mathrm{r}$. 


\section{2 - Estimação para a Distribuição Exponencial Biparamétrica}

Neste caso discute-se os problemas de estimação quando o tempo de vida $T$, pode ser descrito por

$$
\begin{aligned}
f(t ; \theta ; \delta)= & \frac{1}{\theta} \exp \left[-\frac{(t-\delta)}{\theta}\right] ; \quad I_{[0, \infty)}(t) ; \quad \theta>0, \quad \delta \geqslant 0 \\
& \text { EPSTEIN } e \text { SOBEL }(1954) \text { fornecem os estimadores de näxima }
\end{aligned}
$$
verossimilhança para os parâmetros da distribuição exponencial biparamétri ca:

$$
\begin{aligned}
\hat{\delta} & =t_{1} \\
\hat{\theta} & =\frac{\sum_{i=2}^{r}\left(t_{i, n}-t_{1, n}\right)+(n-r)\left(t_{r, I 2}-t_{1, n}\right)}{r} ; \quad r>0 .
\end{aligned}
$$

EPSTEIN (1960) fornece os estimadores imparciais de minnima variância para $\underline{\delta}$ e $\underline{\theta}$

$$
\begin{aligned}
& \tilde{\delta}=t_{1}-\frac{\hat{\theta}}{n} \\
& \tilde{\theta}=\frac{\sum_{i=2}^{r}\left(t_{i, n}-t_{1, n}\right)+(n-r)\left(t_{r, n}-t_{1, n}\right)}{r-1} ; r>1 .
\end{aligned}
$$

o intervalo de confiança para o parâmetro $\underline{\theta}$ e:

$$
\frac{2(r-1) \tilde{\theta}}{x_{2 r+2, \alpha / 2}^{2}} \leqslant \theta \leqslant \frac{2(r-1) \tilde{\theta}}{x_{2 r+2,1-\alpha / 2}^{2}}
$$

MANN (1969) propós estimadores para $\underline{\theta}$ e $\delta$ com menor erro quadrätico entre es estimadores com perda média, os quais são: 


$$
\begin{aligned}
& \hat{\theta}=\bar{t}-t_{1, n}, \\
& \hat{\delta}=\frac{(n+1) t_{1, n}-\bar{t}}{n} .
\end{aligned}
$$

SARHAN (1954), para obter o melhor estimador linear imparcial para $\underline{\delta}$ e $\underline{\theta}$, utiliza estatísticas de ordem e a têcnica de mỉnimos quadrados de LLOYD (1952). Os estimadores de $\underline{\delta}$ e $\underline{\Theta}$ foram:

$$
\begin{aligned}
& \hat{\delta}=\frac{n t_{1, n}-\bar{t}}{n-1}, \\
& \hat{\theta}=\frac{\left(\bar{t}-t_{1, n}\right) n}{n-1} .
\end{aligned}
$$

COHEN e HELM (1973) demonstram que o melhor estimador $1 \underline{i}$ near imparcial, o qual é também um estimador imparcial de mỉnima variância dos parâmetros, pode ser obtido, resolvendo-se o sistema de duas equa ções :

$$
\begin{aligned}
& E(T)=\bar{t}, \\
& E\left(T_{1, n}\right)=t_{1, n},
\end{aligned}
$$

onde $\bar{t} \vec{e}$ a média e $t_{1, n} \vec{e}$ a primeira estatistica de ordem de uma amostra aleatória de tamanho $\mathrm{n}$.

As equações são obtidas aplicando o mëtodo dos momentos modificados, proposto por Helm et ali $i$ (1970).

Para a distribuição exponencial biparamétrica, os valores esperados são:

$$
\begin{aligned}
& E(T)=\delta+\theta=\bar{t}, \\
& E\left(T_{1, n}\right)=\delta+\frac{\theta}{n}=t_{1, n},
\end{aligned}
$$


resolvendo o sistema, obtêm-se os estimadores de $\underline{\theta}$ e $\underline{\delta}$

$$
\begin{aligned}
& \hat{\theta}=\frac{n\left(\bar{t}-t_{1, n}\right)}{n-1}, \\
& \hat{\delta}=\frac{n t_{1, n}-\bar{t}}{n-1} .
\end{aligned}
$$

2.3 - Testes de Hipöteses

0 teste de hipôtese estatîstico $\overline{\mathrm{e}}$ o mais generalizado instrumento da inferência estatỉstica, sendo que, em sua realização, säo apresentadas duas hipōteses, a hipötese nula $\mathrm{H}_{\mathrm{O}}$ e a hipötese alternativa $\mathrm{H}_{1}$.

\subsection{1 - Distribuição Exponencìal Monoparamëtrica}

EPSTEIN (1953) desenvolve um procedimento para testar

$\mathrm{H}_{0}: \theta=\theta_{0}$ versus $\mathrm{H}_{1}: \theta=\theta_{1}<\theta_{0}$. 0 procedimento do teste $\bar{e}$ o seguinte: colocam-se n unidades; após as $x$ primeiras falhas, aceita-se a hipôtese $\mathrm{H}_{\mathrm{O}}$, se $\frac{2 \mathrm{~T}_{\mathrm{r}, \mathrm{n}}}{\theta_{0}}<\mathrm{C}$, onde $\mathrm{T}_{\mathrm{r}, \mathrm{n}}$ é o tempo total do teste, quando a $\underline{r}$-ésima falha ocorre. O trabalho fornece os valores de $\underline{r}$ e C.

O autor, ainda, deduz a förmula para o tempo mẻdio espera do para obter a $\underline{r}$-ésima falha. A förmula ê dada por:

$$
E\left(T_{r, n}\right)=\theta \sum_{i=1}^{r} \frac{1}{n-i+1}
$$

quando a distribuição è exponencial. 
Novamente EPSTEIN (1954-1960) publicou uma outra sërie de complementações a respeito da distribuição exponencial. O autor analisa a substancial melhora nos procedimentos, quando se introduz o procedimento seqüencial. Ele mostra que o teste seqüencial da razão de probabilidades pode ser aplicado aos testes de vida e que, desta forma, as decisões podem ser feitas continuamente no tempo.

BULGREN(1973) propõe um teste de amostragem dupla para testar a média da distribuição exponencial, isto $\vec{e}, H_{0}: \theta<\theta_{0}$ versus $\mathrm{H}_{1}: \theta \geqslant \theta_{1}$. 0 teste proposto é feito em dois estägios e possui o seguinte procedimento: $\underline{n}$ unidades são colocadas em teste; apōs as $\mathrm{r}_{1}$ primeiras faThas aceitam-se a hipótese $H_{0}$, se $\frac{{ }^{2 T_{r_{1}}, n}}{\theta_{0}} \geqslant d_{2}$; rejeita-se a Hipōtese $H_{0}$, se $\frac{2 \mathrm{~T}_{\mathrm{r}_{1}, \mathrm{n}}}{\theta_{0}}<\mathrm{d}_{1}$ e se $\mathrm{d}_{1} \leqslant \frac{2 \mathrm{~T}_{\mathrm{r}_{1}, \mathrm{n}}}{\theta_{0}}<\mathrm{d}_{2}$, deve-se continuar o teste, atē que $r_{2}$ unidades adicionais tenham falhado. Quando $r_{1}+r_{2}=r_{3}$ falhas ocorrem, deve-se aceitar a hipötese $H_{0}$, se $\frac{2 T_{r_{3}, n}}{\theta_{0}} \geqslant d_{3} ;$ se $\frac{2 T_{r_{3}}, n}{\theta_{0}}<d_{3}$ deve-se rejeitar a hipótese $H_{0}$. Bulgren fornece os valores $d_{1}, d_{2}$ e $d_{3} \cdot$ para $\alpha=0,05 ; 0,10$ e $\beta=0,05 ; 0,10$ quando $\theta_{0} / \theta_{1}=2 ; 2,5,3$ e 4 .

\subsection{2 - Distribuição Exponencial Biparamêtrica}

Värios testes da razão de mäxima verossimilhança foram es tudados por NEYMAN. e PEARSON (1928) e SUKHATME (1936) para a distribui- 
ção exponencial biparamëtrica.

PAULSON (1941) estuda a função poder e o viés nos testes de mäxima verossimilhança.

EPSTEIN e TSAO (1953) apresentaram alguns testes para com parar duas distribuiçôes exponenciais. Sejam $n_{1}$ e $n_{2}$ duas amostras aleató rias de duas populações, tendo funções de densidade $f\left(x ; \delta_{1} ; \theta\right)$ e $f\left(y ; \delta_{2} ; \theta\right)$ respectivamente. Os testes fundamentam-se em dois conjuntos de observações $r_{1}$ e $r_{2}$, das amostras de tamanho $n_{1}$ e $n_{2}$ respectivamente. Analisaram os autores os seguintes tipos de testes:

a) Testar $\theta_{1}=\theta_{2}$ supondo $\delta_{1}$ e $\delta_{2}$ conhecidos;

b) Testar $\theta_{1}=\theta_{2}$ supondo $\delta_{1}=\delta_{2}$, porëm desconhecidos;

c) Testar $\theta_{1}=\theta_{2}$;

d) Testar $\delta_{1}=\delta_{2}$ supondo $\theta_{1}$ e $\theta_{2}$ conhecidos;

e) Testar $\delta_{1}=\delta_{2}$ supondo $\theta_{1}=\theta_{2}$, porëm desconhecidos;

f) Testar $\delta_{1}=\delta_{2}$.

Eles fornecem para cada caso, as estatisticas teste e as respectivas regiões crïticas.

KUMAR e PATEL (1971) propōem a estatïstica

$$
\mathrm{T}=\frac{\mathrm{d}\left|\mathrm{X}_{1, \mathrm{n}}-\mathrm{Y}_{1, \mathrm{n}}\right|}{\left(\mathrm{r}_{1}{ }^{-1) \theta_{1}}+\left(\mathrm{r}_{2}{ }^{-1) \theta_{2}}\right.\right.}
$$

onde $d=r_{1}+r_{2}-2$, para testar $H_{0}: \mu_{1}=\mu_{2}$ versus a alternativa $\mathrm{H}_{1}: \mu_{1} \neq \mu_{2}$. A hipōtese $\mathrm{H}_{0} \bar{e}$ rejeitada ao nỉvel de significância $\alpha$, se o valor calcular de $\mathrm{T} \overrightarrow{\mathrm{e}}$ maior que $\mathrm{C}$, onde $\mathrm{P}\left(\mathrm{T}>\mathrm{C} / \mathrm{H}_{\mathrm{O}}\right)=\alpha$. Os valores de C são fornecidos para alguns valores de $r_{1}$ e $r_{Z}$. Para calcular $T$ foram 
utilizados os estimadores de mínima variância das primeiras estatísticas de ordem $X_{1, n}$ e $Y_{1, n}$ e os da vida mëdia $\theta_{1}$ e $\theta_{2}$.

\section{4 - Anālise de Dados}

DAVIS (1952) faz a anãlise de alguns dados de falha. Ver ficou ele que, as falhas dos componentes de um radar, tais como tubos transmissores, "resistores", combinação de transformadores, combinação de "relés", combinação de värios outros componentes, seguen distribuição ex ponencial. A tẻcnica estatística aplicada na anälise dos dados foi o teste qui-quadrado.

Quanto a EPSTEIN (1960), fornece doze procedimentos para testar a validade da- hipótese de que as observações vêm de uma distribuição exponencial. Ele afirma que alguns procedimentos são equivalentes, assim como alguns procedimentos são superiores a outros em certas circunstâncias.

Analisa o autor os seguintes procedimentos:

1) Processo gráfico.

2) Teste qui-quadrado.

3) Um critërio baseado na distribuição condicional da vî da ütil.

4) Teste para verificar se o tempo de falha ê muito curto ou abaixo do normal (falhas precoces).

5) Teste para verificar se o tempo da primeira falha è excessivamente 1 ongo.

6) Teste para detectar a mudança na taxa de falhas. 
7) Teste para verificar se a taxa de falhas pexmanece constante, para dados agrupados em classe.

8) Um caso particular do procedimento 7, para dados não agrupados em classes.

9) Teste baseado na distribuição F máxima.

10) Teste para verificar se os tempos entre falhas são ex cessivamente longos.

11) Procedimento gräfico baseado no teste de Kolmogorov-Smirnov.

12) Teste baseado na taxa de falhas condicional.

MORAN (1951) afirmou que o procedimento 8 (Teste de Bart lett) $\vec{e}$ assintoticamente mais poderoso para o seguinte teste $H_{0}$ " que 0 tempo total entre as sucessivas falhas possui distribuição exponencial versus a hipótese alternativa $\mathrm{H}_{1}$ : que o tempo total entre as sucessivas falhas possui distribuição gama, da forma:

$$
f(t ; \theta, \beta)=\frac{1}{\Gamma(\beta) \theta^{\beta}} t^{\beta-1} \exp \left(\frac{-t}{\theta}\right) ; \quad I_{[0, \infty)}(t), \beta>1, \theta>0 .
$$

BARTHOLOMEW (1957) comparou o procedimento 8 com outros dois procedimentos. Ele verificou que o procedimento $8 \vec{e}$ assintoticamente o mais poderoso dos três procedimentos, quando a hipôtese alternativa $\vec{e}$ Weibul1 ou Gama.

ZELEN e DANNMILLER (1961) examinaram o poder dos procedimentos 3 e 8 , quando a hipótese alternativa $\vec{e}$ Weibull, com função de densidade dada por: 


$$
f(t, \alpha, \beta)=\frac{\beta}{\alpha}\left(\frac{t}{\alpha}\right)^{\beta-1} \exp \left[-\left(\frac{t}{\alpha}\right)^{\beta}\right] ; \quad I_{[0, \infty)}(t), \quad \alpha>0 \text { e } \beta>0 .
$$

Para o procedimento 3 , consideram a situação de censura tipo II sem reposiçâo das unidades que falham; isto $\bar{e}$, o teste começa com $\mathfrak{n}$ unidades e termina quando $\underline{x}$ falhas ocorrem. o tempo total para a i-ésima falha é:

$$
T_{i, n}=T\left(t_{i, n}\right)=t_{1, n}+t_{2, n}+\ldots+(n-i+1) t_{i, n} ; i=1,2, \ldots . r,
$$

is to $\overrightarrow{\mathrm{e}}$ :

$$
T_{1, n}=T\left(t_{1, n}\right)=n t_{1, n}, T_{2, n}=T^{\prime}\left(t_{2, n}\right)=t_{1, n}+(n-1) t_{2, n} \cdots
$$

A distribuição condicional de $T\left(t_{1, n}\right), T\left(t_{2, n}\right), \ldots$, $T\left(t_{r-1, n}\right)$ é uniforme no intervalo $\left[0, T\left(t_{r, n}\right)\right]$ para um dado $T\left(t_{r, n}\right)$. Por tanto:

$$
Z=\frac{\sum_{i=1}^{r-1} T\left(t_{i, n}\right)-\frac{(r-1)}{2} T\left(t_{r, n}\right)}{T\left(t_{r, n}\right) \sqrt{\frac{r-1}{12}}}
$$

é aproximadamente normal com média zero e desvio padrão um.

Deve-se rejeitar a validade da distribulção exponencial ao nivel de significância $\alpha$ se $|z|>z_{\alpha}$, onde $z_{\alpha}$ è o valor crîtico tabelado da distribuição normal.

EPSTEIN (1960) fornece a estatística do procedimento 8:

$B_{r}=\frac{2 r\left\{\ln \left[\frac{T\left(t_{r, n}\right)}{r}\right]-\frac{1}{r}\left[\ln T\left(t_{1, n}\right)+\ln T\left(t_{2, n}-t_{1, n}\right)+\ldots+\ln T\left(t_{r, n}-t_{r-1, n}\right)\right]\right\}}{1+\frac{r+1}{6 r}}$,

onde $T\left(t_{i, n}-t_{i-1, n}\right)$ é o tempo total do intervalo $\left[t_{i-1, n}, t_{i, n}\right]$ e $T_{r, n}=T\left(t_{1, n}\right)+T\left(t_{2, n}-t_{1, n}\right)+\ldots+T\left(t_{r, n}-t_{r-1, n}\right)$, onde $T\left(t_{i, n}-t_{i-1, n}\right)=(n-i+1)\left(t_{i, n}-t_{i-1, n}\right)$. 
Esta estatîstica tem distribuição assintótica de qui-quadcado com r $\quad r$ graus de liberdade. Rejeita-se a validade da distribuição exponencial, se $B_{r}>X_{r-1, \alpha}^{2}$, onde $X_{r-1, \alpha}^{2}$ é o valor crítico tabelado da distribuição de qui-quadrado.

ZELEN e DANNEMILLER (1961) verificaram que, para pequenas amostras, o procedimento 8 não é suficientemente poderoso para distinguir entre as duas distribuiçōes. O procedimento 3 parece ser mais poderoso que o procedimento 8 , quando o parâmetro $\beta$ da distribuição de Weibull for grande. A verificação foi feita por simulação para valores $\beta=1$, $3 / 2,2$ e 3. São necessārias ao menos 20 falhas para o teste ter um razoâvel poder de discriminação.

FERCHO e RINGER (1972) comparam dois testes sugeridos por EPSTEIN (1960) (procedimento 7 e 9) com um procedimento sugerido por GNEDENKO et alii (1960) para amostras censuradas.

A estatistica teste do procedimento 7 è dada por:

$B_{k}=\frac{\left.2 r k\left\{\ln \left[\frac{T\left(t_{k}, r\right.}{k}\right)\right]-\frac{1}{k}\left[\ln T\left(t_{1, r}\right)+\ln T\left(t_{2, r}-t_{1, r}\right)+\ldots+\ln T\left(t_{k, r}-t_{k-1, r}\right)\right]\right\}}{I+\frac{(k+1)}{6 r k}}$

A estatística do procedimento 9 è dada por:

$$
U=\frac{\max \left\{T\left(t_{1, r}\right), T\left(t_{2, r}-t_{1, r}\right), \ldots, T\left(t_{k, r}-t_{k-1, r}\right)\right\}}{\min \left\{T\left(t_{1, r}\right), T\left(t_{2, r}-t_{1, r}\right), \ldots, T\left(t_{k, r}-t_{k-1, r}\right)\right\}}
$$

Tanto no procedimento 7 , como no procedimento 9 , os tempos de falhas são ordenados, tal como:

$$
t_{1,1} \leqslant t_{1,2} \leqslant \ldots \leqslant t_{1, r} \leqslant t_{2,1} \leqslant t_{2,2} \leqslant \ldots \leqslant t_{2, r} \leqslant \ldots \leqslant t_{k, 1} \leqslant \ldots \leqslant t_{k, r}
$$


e agrupados em $\underline{k}$ grupos, cada grupo contendo $\underline{r}$ falhas. 0 tempo total do teste nos intervalos $\left[0, t_{1, r}\right],\left[t_{1, r}, t_{2, r}\right] \cdots\left[t_{k-1, r}, t_{k, r}\right]$ e representado por $T\left(t_{1, r}\right), T\left(t_{2, r}-t_{1, r}\right), \ldots, T\left(t_{k, r}-t_{k-1, r}\right)$ respectivamente.

No procedimento 7 , rejeita-se a hipôtese de que a taxa de falhas não é constante se $B_{k}>x_{k-1, \alpha}^{2}$. O valor crítico $x_{k-1, \alpha}^{2} \vec{e}$ obtido da tabela da distribuição de qui-quadrado.

No procedimento 9, rejeita-se a hipótese de homogeneidade, se $U>F_{\max _{2 r, k, \alpha}}$. HARTLEY (1950) fornece os valores da distribuição $F_{\max }$ para värios valores de $\underline{\mathrm{r}}$ e $\underline{\mathrm{k}}$ adotando $\chi=5 \%$ e $1 \%$.

FERCHO e RINGER (1972), pela anâlise de Monte-Carlo, verí ficaram que o teste de Gnedenko tende a ser mais poderoso, quando a alter nativa è Weibu11.

O teste de Gnedenko estā baseado na seguinte estatística.

$$
Q\left(r_{1}, r_{2}\right)=\frac{\sum_{i=1}^{r} \frac{T\left(t_{i, n}-t_{i-1, n}\right)}{r_{1}}}{\sum_{i=r_{1}+1}^{r} \frac{T\left(t_{i, n}-t_{i-1, n}\right)}{r_{2}}}
$$

sendo $T\left(t_{i, n}-t_{i-1, n}\right)=(n-i+1)\left(t_{i}-t_{i-1}\right)$ com $t_{0}=0 \cdot \operatorname{Cada} T\left(t_{i, n}-t_{i-1, n}\right)$ tem distribuição exponencial e $\sum_{i=1}^{k} \frac{2 T\left(t_{i, n^{-t}}-1, n\right)}{\theta}$ tem distribuição de qui-quadrado com $2 \mathrm{k}$ graus de liberdade e $Q\left(\mathrm{r}_{1}, \mathrm{r}_{2}\right)$ tem distribuição $F$ com $2 r_{1}$ e $2 r_{2}$ graus de 1 iberdade. A distribuição exponencial ë rejeitada, se $Q\left(x_{1}, r_{2}\right) \vec{e}$ maior que $F_{2 r_{1}, 2 r_{2}, \alpha / 2}$, ou menor que $F_{2 x_{1}}, 2 r_{2}, 1-\alpha / 2$. 
Quando $Q\left(r_{1}, r_{2}\right)$ è grande, isto indica que a taxa de fa1has esta aumentando; quando pequeno, a taxa de fâlhas estâ diminuindo. Fercho e Ringer utilizaram $r_{1}=r_{2}=\frac{r}{2}$ para $\underline{r}$ par e $r_{1}=\left[\frac{r}{2}\right] ; r_{2}=\left[\frac{r}{2}\right]+1$ para Impar, onde [ ] indica o maior inteiro contido.

Para verificar a validade do modelo exponencial, pode-se tambēm utilizar o teste de Kolmogorov-Smirnov. A estatistica teste para um teste bilateral é:

$$
D_{n}=\operatorname{máx}\left(\delta_{i}\right),
$$

onde: $\quad \delta_{i}=\operatorname{máx}\left[\frac{i}{n}-F\left(t_{i} ; \theta\right) ; F\left(t_{i} ; \theta\right)-\frac{(i-1)}{\theta}\right]$, $\operatorname{com} F\left(t_{i} ; \theta\right)=1-\exp \left(\frac{-t_{i}}{\theta}\right) ; \quad i=1,2, \ldots, n ;$ onde $t_{i} \vec{e}$ a $\underline{i}-\hat{e}$ esima estatística de ordem.

Para a distribuição exponencial com mêdia desconhecida, LILLIEFORS (1969) fornece os valores crîticos para a estatística de Kolmogorov-Smirnov, quando a amostra ê completa. 
3. FUNDAMENTOS TEORICOS

3.1 Propriedades Estatỉsticas da Distribuição Exponencial

A função de densidade da variâvel aleatória exponencial T com dois parâmetros tem a forma:

$$
f(t ; \theta, \delta)=\frac{1}{\theta} \exp \left[\frac{-(t-\delta)}{\theta}\right] ; I_{[\delta, \infty)}(t), \theta>0, \delta \geqslant 0 .
$$

Para (3.1.a) tem-se a seguinte denominação para os parâmetros:

$\delta$ : parâmetro de localizaçâo, de vida minima, de garantia ou de "soleira".

$\theta$ : parâmetro de escala ou vida mêdia.

A função distribuição ê obtida de (3.1.a) atravês de integração: 
$F(t ; \theta, \delta)=P(T \leqslant t)=1-\exp \left[\frac{-(t-\delta)}{\theta}\right] ; I_{[. \delta, \infty)}(t) ; \theta>0, \quad \delta \geqslant 0$.

0 valor esperado e a variância são $\theta+\delta$ e $\theta^{2}$ respectivamente.

Como a distribuição biparamêtrica pode ser sempre convertida em uma distribuição monoparamētrica, fazendo-se a translação $T=t-\delta$, ter $-\operatorname{se}-\vec{a}$ de $(3.1 \cdot a)$

$$
f(t ; \theta)=\frac{1}{\theta} \exp \left(\frac{-t}{\theta}\right) ; \quad I_{[0, \infty)}(t) ; \quad \theta>0
$$

Integrando a (3.1.c), obtëm-se a função de distribuição:

$$
F(t ; \theta)=P(T \leqslant t)=1-\exp \left(\frac{-t}{\theta}\right) ; I_{[0, \infty)}(t), \theta>0
$$

A função de confiabilidade para (3.1.a) ê dada por:

$C(t ; \theta, \delta)=P(T>t)=\exp \left[\frac{-(t-\delta)}{\theta}\right] ; I_{[\delta, \infty)}(t), \theta>0, \quad \delta \geqslant 0$.

e para (3.1.c) è:

$$
C(t ; \theta)=P(T>t)=\exp \left(\frac{-t}{\theta}\right) ; \quad I_{[0, \infty)}(t), \quad \theta>0
$$

A taxa de falhas é a probabilidade de que uma falha por unidade de tempo ocorra no intervalo $\left[t_{1}, t_{2}\right]$, dado que não ocorreu atē o instante $t_{1}$.

Portanto, a taxa de Ealhas ề dada por: 


$$
\frac{P\left(t_{1} \leqslant T \leqslant t_{2}\right) /\left(t_{2}-t_{1}\right)}{C\left(t_{1}\right)}=\frac{C\left(t_{1}\right)-c\left(t_{2}\right)}{\left(t_{2}-t_{1}\right) C\left(t_{1}\right)} .
$$

Como se vê, a taxa de falhas ê uma função do tempo.

Ao redefinir-se o intervalo para $[t, t+\Delta t]$, a expressão

(3.1.g) ficarā:

$$
\frac{C(t)-C(t+\Delta t)}{\frac{C(t)}{\Delta t}}
$$

A taxa de falhas definida en (3.1.h) ê expressa em falhas por unidade de tempo. Na realidade, as unidades de tempo pódem ser quilometros, rotações, ciclos, horas, e assim por diante.

A função intensidade de falhas ou funçâo acaso ê definida como o limite da taxa de falhas, quando o intervalo aproxima-se de zero. Da expressão $(3.1 . h)$ ter-se-ä:

$$
h(t)=\lim _{\Delta t \rightarrow 0} \frac{C(t)-C(t+\Delta t)}{\Delta t C(t)}=\frac{1}{C(t)}\left[-\frac{d}{d r} C(t)\right]=
$$

como $-\left[\frac{d}{d t} C(t)\right]=f(t), \quad$ então: $h(t)=\frac{f(t)}{C(t)}$.

Fortanto, a função acaso è a taxa de falhas instantânea.

A taxa de falhas instantânea para (3.1.a) e (3.1.c) ê uma constante, ou seja, è dada por:

$$
h(t)=\frac{1}{\theta}
$$


Uma outra propriedade importante da distribuição exponencial è dada por:

$$
\begin{aligned}
P(T>t+a \mid T>t) & =\frac{\exp \left[\frac{-1}{\theta}(t+a)\right]}{\exp \left(\frac{-t}{\theta}\right)}, \\
& =\exp \left(\frac{-a}{\theta}\right) .
\end{aligned}
$$

Como se vê, de (3.1.k), a probabilidade desejada $\vec{e}$ independente do tempo $t$, tempo que uma unidade sobreviveu antes do intervalo de tempo a. Fisicamente, isto significa que enquanto o componente estiver funcionamento ele será "tão bom quanto novo", e, por isso, seu comportamento de falhas durante qualquer perïodo particular depende sómente da extensão desse perỉodo e não do seu passado as experiêricias do sistema não influenciam quanto ao desgaste; esta è uma propriedade fundamental da distribuição exponencial.

Por sua vez, a distriouição exponencial e a distribuição de Poisson estão diretamente relacionadas. Se o tempo entre falhas ë uma variável aleatōria $\mathrm{T}$ com distribuição exponencial com parâmetro $\underline{\theta}$, então o nümero de falhas em um intervalo de tempo $t$, possui distribuição de Poisson com parâmetro $t / \theta$, ou seja, se:

$$
t(t ; \theta)=\frac{1}{\theta} \exp \left(\frac{-t}{\theta}\right) ; \quad I_{[0, \infty)}(t), \quad \theta>0
$$

onde $\mathrm{T}$ é a variável tempo entre falhas, então, para um intervalo de tempo de duração t, tem-se:

$$
P(X=r)=\frac{\left(\frac{t}{\theta}\right)^{r} \exp \left(\frac{-t}{\theta}\right)}{r !} ; \quad I_{\{0,1,2, \ldots\}}(r),
$$

onde $\underline{e}$ e nümero de falhas observadas no intervalo de tempo de duração t. 
Para demonstrar (3.1.m), supöem-se que X seja o nümero de falhas no intervalo de tempo $[0, t]$ e que $T_{r}$ è o tempo da $\underline{r}$-ésima fa1ha.

Deve-se observar que se tem $x<r$, se, e somente se,

$\mathrm{T}_{\mathrm{r}}>\mathrm{t} \cdot \mathrm{Ou}:$

$$
\begin{aligned}
& P(X<r)=P\left(T_{r}>t\right), \\
& P(X<r+1)=P\left(T_{r+1}>t\right) .
\end{aligned}
$$

Portanto,

$$
P(X=r)=P\left(T_{r+1}>t\right)-P\left(T_{r}>t\right) .
$$

Dai, tem-se:

$$
\begin{aligned}
& P(X=r)=\left[\begin{array}{l}
\left.1-P\left(T_{r+1} \leqslant t\right)\right]-\left[1-P\left(T_{r} \leqslant t\right)\right], \\
P(X=r)=P\left(T_{r} \leqslant t\right)-P\left(T_{r+1} \leqslant t\right), \\
P(X=r)=F_{T_{r}}(t)-F_{T_{r+1}}(t) .
\end{array}\right. \\
& \text { Mas, } T_{r}=t_{1}+t_{2}+\ldots+t_{r} \text {, onde os } t_{i} \text { são inde }
\end{aligned}
$$

pendentes e distribuĩdos exponencialmente. A função geradora de momentos para $\mathrm{T}_{\mathrm{r}} \quad \overrightarrow{\mathrm{e}}$ dada por:

$$
\mathrm{M}_{\mathrm{T}}(\mathrm{s})=\left[\mathrm{M}_{\mathrm{T}}(\mathrm{s})\right]^{\mathrm{r}}
$$

Como a função geradora de momentos da distribuição exponencial $\bar{e} M_{T}(s)=\frac{1}{1-\theta s} ; s<\frac{1}{\theta}$, tem-se, portanto, que:

$$
\mathrm{M}_{\mathrm{T}_{\mathrm{r}}}(\mathrm{s})=\left(\frac{1}{1-\theta \mathrm{s}}\right)^{\mathrm{r}} ; \mathrm{s}<\frac{1}{\theta} .
$$


Da expressão (3.1.r) pode-se verificar que $\mathrm{T}_{\mathrm{r}}$

possui distribuiçāo gama, cuja função densidade, è:

$$
f\left(T_{r} ; \theta, r\right)=\frac{1}{\theta^{r}(r-1) !} T_{r}^{r-1} \exp \left(\frac{-T_{r}}{\theta}-\right) ; \quad I_{(0, \infty)}\left(T_{r}\right), \theta>0 .(3.1 . s)
$$

Integrando-se (3.1.s) tem-se:

$$
F_{T_{r}}(t ; \theta, r)=\frac{1}{\theta^{r}(r-1) !} \int_{0}^{t} T_{r}^{r-1} \exp \left(\frac{-T_{r}}{\theta}\right) d T_{r} \cdot \text { (3.1.t) }
$$

Aplicando integração por partes, obtëm-se um modelo que e:

$F_{T_{r}}(t ; \theta, r)=-\exp \left(-\frac{t}{\theta}\right)\left[\frac{1}{(r-1) !}\left(\frac{t}{\theta}\right)^{r-1}+\frac{1}{(r-2) !}\left(\frac{t}{\theta}\right)^{r-2}+\ldots+1\right]+1 \cdot(3 \cdot 1 \cdot u)$ Analogamente,

$\mathrm{F}_{\mathrm{T}+1}(\mathrm{t} ; \theta, r)=-\exp \left(-\frac{1}{\theta}\right)\left[\frac{1}{\mathrm{r} !}\left(\frac{\mathrm{t}}{\theta}\right)^{\mathrm{r}}+\frac{1}{(\mathrm{r}-1) !}\left(\frac{t}{\theta}\right)^{r-1}+\ldots+1\right]+1$.

Substituindo (3.1.u) e (3.1.v) em (3.1.p), ter-se-á finalmente:

$$
P(X=r)=\frac{\left(\frac{t}{\theta}\right)^{r} \exp \left(-\frac{t}{\theta}\right)}{r !} ; \quad I_{\{0,1,2, \ldots\}}(r)
$$

E interessante verificar que as distribuições exponencial, gama, de Rayleigh, de Erlang e de Weibull pertencem à "família" cuja função de densidade ê dada por: 
$f(x ; \alpha, \beta)=A(\alpha, \beta) x^{\alpha-1} \exp \left[-B(\alpha, \beta) x^{\beta}\right] ; \quad I_{[0, \infty)}(x), \quad \alpha>0, \quad \beta>0$.

Assim, se $\alpha=\beta=1$, ter-se-á a distribuição exponencial, se $\alpha>0, \beta=1$, a distribuição gama, se $\alpha=\beta=2$, a distribuição de Rayleigh; se $\alpha=2$ e $\beta=1$, a distribuição de Erlang e se $\alpha=\beta>0$, a distribuição de Weibul1.

3.2 Estimação para a Distribuição Exponencial

Monoparamëtrica

\subsection{1 - Censura Tipo II: Amostragem sem reposição}

o processo de estimação que serä desenvolvido é a técnica de mäxima verossimilhança.

Para ñ falhas ordenadas, tem-se a função de verossimilhan ça:

$$
L\left(t_{1, n}, t_{2, n}, \ldots, t_{n, n} ; \theta\right)=n ! \prod_{i=1}^{n} f\left(t_{i, n} ; \theta\right) \cdot \quad \text { (3.2.1.a) }
$$

Para a distribuição exponencial a (3.2.1.a), torna-se:

$L\left(t_{1, n}, t_{2, n} \cdots, t_{n, n} ; \theta\right)=n ! \frac{1}{\theta^{n}} \exp \left(\frac{-\sum_{i=1}^{n} t_{i, n}}{\theta}\right) . \quad$ (3.2.1.b) A ordenação não faz nenhuma diferença, desde que todas as n unidades sejam mantidas, ate ocorrer a falha. Maximizando (3.2.1.b), ter se- $-\bar{a}:$ 


$$
\hat{\theta}=\frac{\sum_{i=1}^{n} t_{i, n}}{n}=\bar{t} .
$$

Este estimador deve ser utilizado, quando a amostra é com pleta, isto $\vec{e}$, o experimento termina quando todas as unidades falham.

Contudo, se pararmos o teste apös a $r$-Ésima falha, quando $\mathrm{r}<\mathrm{n}$, observam-se as unidades mais fracas da amostra. Neste caso, a ordenação deve ser considerada, e a função de verossimjihança ficarä:

$$
\begin{array}{r}
L\left(t_{1, n}, t_{2, n}, \ldots, t_{r, n} ; \theta\right)=\frac{n !}{(n-r) !}\left[\frac{1}{\theta} \exp \left(\frac{-t_{1, n}}{\theta}\right) \cdot \frac{1}{\theta} \exp \left(\frac{-t_{2}, n}{\theta}\right) \ldots\right. \\
\ldots \exp \left[\frac{\left.-(n-r) t_{r, n}\right]}{\theta}\right],
\end{array}
$$

ou:

$$
\begin{gathered}
L\left(t_{1, n}, t_{2, n}, \ldots, t_{r, n} ; \theta\right)=\frac{n !}{(n-r) !} \cdot \frac{1}{\theta r} \exp \left\{\frac{-\left[\sum_{i=1}^{r} t_{i, n}+(n-r) t r, n\right]}{\theta}\right\} ; \\
\left.\quad 0<t_{1, n} \leqslant t_{2, n} \leqslant \ldots \leqslant t_{r, n} .1 . d\right)
\end{gathered}
$$

Tomando-se o logaritmo natural da expressão (3.2.1.d), de rivando-se em relação a $\underline{\theta}$ e igualando-se a zero, obtēm-se o estimador de E que maximiza (3.2.1.d). Este estimador $\overrightarrow{\mathrm{e}} \mathrm{dad}$ o por:

$$
\hat{\theta}=\frac{\sum_{i=1}^{r} t_{i, n}+(n-r) t_{r, n}}{r} ; r>0 .
$$

Como a função de verossimilhança (3.2.1.d) pode ser fatorada como $g(\hat{\theta}, \theta) h\left(t_{1, n}, t_{2, n}, \ldots, t_{r, n}\right)$, o estimador para $\underline{\theta} \bar{e}$ suficiente. 
Para verificar que $\hat{\theta} \vec{e}$ um estimador imparcial, é necessärio conhecer a distribuição de $\hat{\theta}$.

Se definirmos novas variäyeis:

$$
Y_{i, n}=T_{i, n}-T_{i-1, n} \quad i=1,2, \ldots, r \text { e } t_{a}=0,(3.2 .1 . f)
$$

como:

$$
T_{i, n}=\sum_{j=1}^{i} t_{j, n}+(n-r) t_{i, n},
$$

em função de $Y_{i}$ ficară:

$$
T_{r, n}=T\left(t_{r, n}\right)=n t_{1}+(n-1)\left(t_{2, n}-t_{1, n}\right)+\ldots+(n-r+1) t_{r, n} \text {, }
$$

ou

$$
T_{r, n}=T\left(t_{r, n}\right)=\sum_{i=1}^{r}(n-i+1) y_{i, n} .
$$

As variāveis aleatỏrias $Y_{i}$ são mutuamente independentes. o módulo do Jacobiano para essa transformação (um a um) é um. Substituin do em $(3.2 .1 . d)$, ter-se-ä:

$$
\begin{aligned}
& L\left(y_{1, n}, y_{2}, n, \ldots, y_{r, n} ; \theta\right)=\frac{n !}{(n-r) !} \frac{1}{\theta} \exp \left[\frac{-\sum_{i=1}^{r}(n-i+1) y_{i, n}}{\theta}\right] ; \\
& I_{[0, \infty)}\left(y_{i, n}\right), \quad \theta>0 .
\end{aligned}
$$

A distribuição marginal para $Y_{k, n}$ pode ser obtida pela in tegração de $(3.2 .1 . h),(r-1)$ vezes. Integrando-se para um $Y_{\mathrm{p}, \mathrm{r}^{3}}$, tem-se: 
28.

$$
\begin{aligned}
& h\left(y_{k, n} ; \theta\right)=\frac{n !}{(n-r) ! \theta^{r}} \int_{0}^{\infty} \int_{0}^{\infty} \cdots \int_{0}^{\infty} \exp \left[\frac{\begin{array}{c}
i \neq 1 \\
- \\
i \neq p
\end{array}}{\theta}\right] \\
& \cdot \exp \left[\frac{-(n-p+1) y_{p, n}}{\theta}\right] \underset{\substack{i=1 \\
i \neq p}}{\left(\prod_{i, n}^{r} d y_{p, n}\right) d y_{p}} \\
& \text { Se } u=\frac{(n-p+1) y_{p, n}}{\theta} \text {, então, } d u=\frac{(n-p+1) d y p, n}{\theta} \text {; substi- }
\end{aligned}
$$
tuindo essas expressöes em (3.2.1.i), ter-se-ä:

$$
\begin{aligned}
& h\left(y_{k, n} ; \theta\right)=\frac{n !}{(n-r) !(n-p+1) \theta^{r-1}} \int_{0}^{\infty} \int_{0}^{\infty} \cdots \int_{0}^{\infty} \exp \left[\frac{-\sum_{i \frac{1}{1}}^{r}(n-i+1) y p, n}{\theta}\right] . \\
& \left.\prod_{\substack{i \neq 1 \\
i \neq \bar{p}}}^{x} d y_{i, n}\left[-e^{-u}\right]\right|_{0} ^{\infty} \text {, }
\end{aligned}
$$

ou :

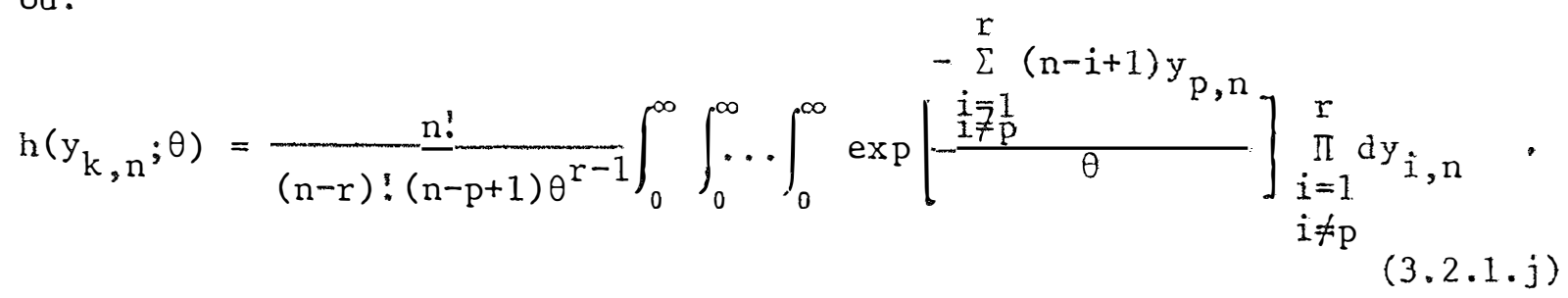$$
\text { Deve-se observar que } \frac{n !}{(n-r) ! \cdot(n-p+1)} \text { reduz-se a }
$$
$(n-k+1)$, quando todas as $Y_{k, n}$ são integradas. Portanto, a diștribuição marginal para $\mathrm{Y}_{\mathrm{k}, \mathrm{n}}$ torna-se, após integrar $(\mathrm{r}-1)$ vezes:$$
h\left(y_{k, n} ; \theta\right)=\left(\frac{n-k+1}{\theta}\right) \exp \left[\frac{-(n-k+1) y_{k}, n}{\theta}\right] ; \quad I_{[0, \infty)}\left(y_{k, n}\right), \quad \theta>0 .
$$ 
Quando as variäveis são independentes, ter-se-ä:

$$
f\left(y_{1, n}, y_{2, n}, \ldots, y_{x, n}\right)=h\left(y_{I, n}\right) h\left(y_{2, n}\right) \ldots h\left(y_{x, n}\right)
$$

e, nesse caso,

$$
\begin{aligned}
L\left(y_{1, n}, y_{2}, n, \ldots, y_{r, n}\right) & =\prod_{i=1}^{r}\left(\frac{n-i+1}{\theta}\right) \exp \left[\frac{-(n-i+1) y_{i, n}}{\theta}\right] . \\
& =\frac{n !}{(n-r) !} \cdot \frac{1}{\theta^{r}} \exp \left[\frac{\sum_{i=1}^{r}(n-i+1) y_{i, n}}{\theta}\right] .
\end{aligned}
$$

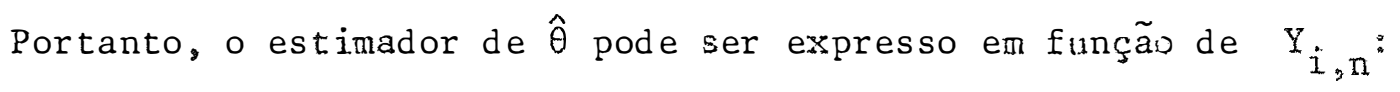

$$
\hat{\theta}=\frac{\sum_{i=1}^{r}(n-i+1) y_{i, n}}{r} ; r>0,
$$

Pode-se agora encontrar a distribuição de $\hat{\theta}, 1$ enbrando-se que as variâveis $Y_{\dot{j}, n}$ são independentes e utilizando-se a função geradora de momentos para a distribuição de $\hat{\theta}$.

Visto que a variävel ${ }^{\mathrm{x}} \mathrm{i}, \mathrm{n}$ possui distribuição exponencial, a função geradora de momentos ficarä:

$$
\begin{aligned}
& M_{Y_{i, n}}(s)=\frac{1}{1-\left(\frac{\theta}{n-i+1}\right) s} ; \quad s<\frac{n-i+1}{\theta}, \\
& \text { Então, } \\
& M_{\hat{\theta}}(s)=M_{Y_{1, n}}\left(\frac{n s}{r}\right) \cdot M_{Y_{2, n}}\left[\frac{(n-1) s}{r}\right] \ldots M_{Y_{r, n}}\left[\frac{(n-r+1) s}{r}\right],
\end{aligned}
$$


ou:

$$
M_{\hat{\theta}}(s)=\left[\frac{1}{1-\left(\frac{\theta}{r}\right) s}\right]^{r} ; \quad s<\frac{r}{\theta}
$$

A expressão (3.2.1.p) è a função geradora de momentos da distribuição gama, portanto, isto implica em que:

$f(\hat{\theta} ; \theta, r)=\frac{1}{(r-1) !}\left(\frac{r}{\theta}\right)^{r} \hat{\theta}^{r-1} \exp \left(\frac{-\hat{\theta} r}{\theta}\right) ; \quad I_{[0, \infty)}(\hat{\theta}) ; \quad \theta>0, \quad r>0$.

Pode-se verificar que $E(\hat{\theta})=\theta$; portanto $\hat{\theta} \vec{e}$ um estinador imparcial de $\theta$. Tem-se, tambēm, que $V(\hat{\theta})=\frac{\theta^{2}}{r}$ sendo igual ao 1 imite inferior de Cramer-Rao. Assim $\hat{\theta}$ tem variância mỉnima e é eficiente.

De (3.2.1.q) pode-se calcular os limites de confiança para $\hat{\theta}$. Entretanto, existe uma grande vantagem em poder utilizar uma distribuição jā tabelada. A função de densidade de uma variāvel aleatória com distribuição qui-quadrado é:

$f(x ; \theta, k)=\frac{1}{2^{k / 2} T(k / 2)} x^{k / 2-1} \exp \left(\frac{-x}{2}\right) ; \quad I_{[0, \infty)}(x), \quad k=1,2, \ldots$.

Fazendo-se $\frac{r \hat{\theta}}{\theta}=\frac{x}{2}$, entrão $d \hat{\theta}=\frac{\theta}{2 r} d x$. Substituindo estas expressões em $(3.2 .1 .9)$, tem-se:

$f(x ; \theta, r)=\frac{1}{2^{r}} \frac{1}{(r-1) !} x^{r-1} \cdot \exp \left(\frac{-x}{2}\right) ; \quad I_{[0, \infty)}(x), \quad r=1,2,3, \ldots$. $(3.2 .1 .5)$ 
graus de liberdade. Assim,

$$
\mathrm{p}\left(x_{2 r, 1-\alpha / 2}^{2} \leqslant \frac{2 r \hat{\theta}}{\theta} \leqslant x_{2 r, \alpha / 2}^{2}\right)=1-\alpha .
$$

Portanto,o intervalo de confiança para $\underline{\theta}$ serä:

$$
P\left(\frac{2 r \hat{\theta}}{x_{2 r, \alpha / 2}^{2}} \leqslant \theta \leqslant \frac{2 r \hat{\theta}}{x_{2 r, 1-\alpha / 2}^{2}}\right)=1-\alpha .
$$

Quando a amostra é completa, isto é, todas as unidades fa Iharam, o intervalo de confiança para $\theta$ pode ser obtido de (3.2.1.t), fazendo-se, $r=n$.

Pode-se, também, determinar o comprimento do intervalo de $(3.2 .1 . t):$

$$
L=2 r \hat{\theta}\left(\frac{1}{x_{2 r, 1-\alpha / 2}^{2}}-\frac{1}{x_{2 r, \alpha / 2}^{2}}\right) \quad
$$

Como $\hat{\theta} \vec{e}$ uma variâvel aleatória, o comprimento do interva 1o é também uma variâvel e o comprimento esperado serâ obtido de (3.2.1.u), substituindo-se $\hat{\theta}$ por $\underline{\theta}$.

\subsection{2 - Censura Tipo II - Amostragem com reposiç̧ão}

Quando a amostragem ê com reposição, as unidades que faIham são repostas. 0 experimento permanece, sempre, com $\underline{n}$ unidades, terminando após a r-ësima falha. 
A função de verossimilhança, para os tempos de falhas ordenados, è dada por:

ou

$$
L\left(t_{1, n}, t_{2, n}, \ldots, t_{r, n} ; \theta\right)=\left(\frac{n}{\theta}\right)^{r} \exp \left[\frac{-n \sum_{i=1}^{r}\left(t_{i, n}-t_{i-1, n}\right)}{\theta}\right],
$$

$$
\begin{array}{r}
L\left(t_{1, n}, t_{2, n}, \ldots, t_{r, n} ; \theta\right)=\left(\frac{n}{\theta}\right)^{r} \exp \left(\frac{-n t_{r, n}}{\theta}\right), \\
0 \leqslant t_{1, n} \leqslant t_{2, n} \leqslant \ldots \leqslant t_{r, n} ; t_{0}=0 .
\end{array}
$$

E para obter-se o estimador de māxima verossimilhança, deve-se tomar o logaritmo natural da expressão (3.2.2.a), derivar em rela ção a $\underline{\theta}$ e igualar a zero. 0 estimador $\bar{e}$ dado por:

$$
\hat{\theta}=\frac{n t}{r} r, r>0 .
$$

Se definirmos uma nova variävel $Y_{i, n}$ como sendo a diferença entre falhas então

$$
Y_{i, n}=T_{i, n}-T_{i-1, n} ; t_{0}=0 \text {. }
$$

Pode-se reescrever, assim, a expressão (3.2.2.a) como:

$$
\begin{gathered}
L\left(t_{1, n}, t_{2, n}, \ldots, t_{r, n} ; \theta\right)=\left(\frac{n}{\theta}\right)^{r} \exp \left(\frac{-\sum_{i=1}^{-n} y_{i, n}}{\theta}\right) ; I_{(0, \infty)}\left(y_{i, n}\right), \\
0 \leqslant y_{1, n} \leqslant y_{2, n} \leqslant \ldots \leqslant y_{r, n} .
\end{gathered}
$$


Cada $Y_{i, n}$ possui distribuição exponencial com parâmetro n/ $\theta$. o môdulo do jacobiano dessa transformação (um a um) $\vec{e}$ um.

Então, $T_{r, n}=\sum_{i=1}^{r} Y_{i}$ possui distribuição gama, cuja função de densidade è dada por:

$f\left(t_{r, n} ; \theta, r\right)=\frac{1}{\Gamma(r)}\left(\frac{n}{\theta}\right)^{r} t_{r, n}^{r-1} \exp \left(\frac{-n t_{r, n}}{\theta}\right) ; \quad I_{[0, \infty}\left(t_{r, 1 x}\right), \quad \theta>0, \quad r>0$.

Conseqüentemente, $\frac{{ }^{2 n t} r, n}{\theta}$ possui distribuição de qui-quadra do com $2 r$ graus de liberdade. Assim, o intervalo de confiança para $\underline{\theta} \vec{e}:$

então

$$
\mathrm{P}\left(x_{2 r, 1-\alpha / 2}^{2} \leqslant \frac{2 \mathrm{nt} r, \mathrm{n}}{\theta} \leqslant x_{2 r, \alpha / 2}^{2}\right)=1-\alpha
$$

$$
\mathrm{P}\left(\frac{2 \mathrm{nt} r, \mathrm{n}}{x_{2 r, \alpha / 2}^{2}} \leqslant \theta \leqslant \frac{2 \pi t_{r, n}}{x_{2 r, 1-\alpha / 2}^{2}}\right)=1-\alpha,
$$

Ou:

$$
\mathrm{P}\left(\frac{2 r \hat{\theta}}{x_{2 r, \alpha / 2}^{2}} \leqslant \theta \leqslant \frac{2 r \hat{\theta}}{x_{2 r, 1-\alpha / 2}^{2}}\right)=1-\alpha
$$

\subsection{3 - Censura Tipo I - Amostragem sem reposição}

Colocam-se n unidades em teste e o mesmo termina para um prefixado tempo $\underline{t}^{*}$. A função de verossimilhança para este caso é: 


$$
\begin{aligned}
L\left(t_{1, n}, t_{2, n}, \ldots, t_{r, n} ; \theta\right) & =\frac{n !}{(n-r) !} \frac{1}{\theta^{r}} \exp \left\{\frac{-\left[\sum_{i=1}^{r} t_{i, n^{*}(n-r) t^{*}}\right]}{\theta}\right\} \\
0 & <t_{1, n} \leqslant t_{2, n} \leqslant \cdots \leqslant t_{r, n}<t^{*} .
\end{aligned}
$$

Para obter-se o estimador de mäxima verossimilhança de $\underline{\theta}$, deve-se tomar o logaritmo natural da expressão (3.2.3.a), derivar em rela ção a $\underline{\theta}$ e igualar a zero. Este estimador ē dado por:

$$
\hat{\theta}=\frac{\sum_{i=1}^{r} t_{i, n}+(n-r) t^{*}}{r} ; r>0 .
$$

o estimador obtido em (3.2.3.b) não ê imparcial, pois $E(\hat{\theta}) \neq \theta$

Un outro estimador de $\underline{\theta}$ pode ser obtido; supõe-se que uma estimativa da proporção de falhas è dada por:

$$
\mathrm{p}=\frac{\mathrm{r}}{\mathrm{n}}
$$

A probabilidade de que uma unidade falhe, antes que o experimento termine $\bar{e}$ :

$$
p=1-\exp \left(\frac{-t^{*}}{\theta}\right)
$$

Das expressões $(3.2 .3 . c)$ e (3.2.3.d), tem-se:

$$
\widetilde{\theta}=\frac{-t^{*}}{\ln \left(1-\frac{r}{n}\right)}=\frac{-t^{*}}{\ln (1-p)} .
$$


BARTOLOMEW (1963) compara $\hat{\theta}$ e $\tilde{\theta}$, determinando o limite da eficiência relativa que $\hat{e}$ dado por:

$$
\lim _{n \rightarrow \infty} \frac{V(\hat{\theta})}{V(\tilde{\theta})}=\frac{(1-p)[\ln (1-p)]^{2}}{p^{2}} .
$$

Ele verificou que o estimador de mäxima verossimilhança $\underline{\theta}$ é preferível, porém $\tilde{\theta}$ è bastante bom para valores de $\underline{p}$ pequenos. A eficiência relativa excede a $96 \%$, quando $p \leqslant 0,5$.

Neste caso, é muito difícil obter os limites de confiança para o parâmetro $\underline{\theta}$. Uma aproximação estā baseada na distribuição binomial, que utiliza a informação de que $\underline{r}$ falhas ocorrem no intervalo do teste $\left[0, t^{*}\right]$, quando $\underline{n}$ unidades são testadas.

A probabilidade de que $\underline{r}$ unidades falhem, at $\vec{e} \circ$ tempo $\underline{t}^{*}, \vec{e}$ dada por:

$$
P(X=r)=\left({ }_{r}^{n}\right) p^{r}(I-p)^{n-r} ; I_{\{a, 1,2, \ldots, n\}}(x) .
$$

Os limites de confiança para $\underline{p}$ são obtidos, resolvendo-se as duas equações. O limite de confiança superior de $\underline{p} \vec{e}$ obtido pela solução da equação:

$$
P(x \leqslant r)=\sum_{x=0}^{r}\left(\begin{array}{l}
n \\
x
\end{array}\right) p_{s}^{x}\left(1-p_{s}\right)^{n-x}=\frac{\alpha}{2} .
$$

o limite de confiança inferior de $\underline{p}$ ê obtido pela solução da equação: 


$$
P(X<r)=\sum_{x=0}^{r-1}\left(\frac{n}{x}\right) P_{I}^{x}\left(1-p_{I}\right)^{n-x}=1-\frac{\dot{\alpha}}{2} .
$$

Assim, o intervalo de confiança para pe ê:

$$
P\left(p_{I} \leqslant p \leqslant p_{s}\right)=1-\alpha
$$

Substituindo-se $(3 \cdot 2 \cdot 3 \cdot e)$ em $(3 \cdot 2 \cdot 3 \cdot j)$, ter-se-âa:

$$
P\left[\frac{-t^{*}}{\ln \left(1-p_{s}\right)} \leqslant \theta \leqslant \frac{-t^{*}}{\ln \left(1-p_{I}\right)}\right]=1-\alpha .
$$

- limite de confiança inferior $\underline{\theta}$, ao nỉvel de probabilidade $1-\alpha$ é dado por:

$$
P\left[\theta_{I} \geqslant \frac{-t^{*}}{\ln \left(1-p_{s}\right)}\right]=1-\alpha
$$

onde $\underline{p}_{s} \bar{e}$ obtido de:

$$
\sum_{x=0}^{r}\left({ }_{x}^{n}\right) p_{s}^{x}\left(1-p_{s}\right)^{n-x}=\alpha
$$

Um outro limite de confiança inferior para $\underline{p}$, $\vec{e}$ dado pela solução da equação:

$$
\sum_{x=0}^{r-1}\left({ }_{x}^{n}\right) p_{I}^{x}\left(1-p_{I}\right)^{n-x}=1-\alpha=\frac{\Gamma(n+1)}{\Gamma(n-r) \Gamma(r+1)} \int_{0}^{p_{I}} y^{n-r-1}(1-y)^{r} d y \cdot(3 \cdot 2 \cdot 3 \cdot m)
$$

Da relação entre a distribuição binomial e a distribuição beta, tem-se:

$$
\mathrm{P}_{\mathrm{I}}=1-\text { Beta }_{\nu_{1}, v_{2}, 1-\alpha}=\operatorname{Beta}_{\nu_{2}, \nu_{1}, \alpha,}
$$

onde

$$
\nu_{1}=r+1 \quad \text { e } \quad v_{2}=n-r
$$


Os percentis da distribuição podem ser calculados, utilizando-se a distribuição $F$, atravês da seguinte relação:

$$
\text { Beta }_{v_{1}, v_{2}}=\frac{\left(\frac{v_{1}}{v_{2}}\right) F_{2 \nu_{1}}, 2 v_{2}}{1+\left(\frac{v_{1}}{v_{2}}\right) F_{2 v_{1}}, 2 v_{2}}
$$

\subsection{4 - Censura Tipo I - Amostragem com reposição}

Considerem-se In unidades, as mesmas serão repostas, à medida que falharem; o teste é censurado após um específico tempo $\underline{t}^{*}$. 0 experimento permanece sempre com $\underline{n}$ unidades e o tempo total do teste $\hat{e}$ $n t^{*}$.

A variävel número de falhas possui distribuição de Poisson, com parâmetro $n t^{*} / \theta$.

A função de probabilidade da distribuição de Poisson è dada por:

$$
P(X=x)=\frac{\left(\frac{n t^{*}}{\theta}\right)^{x} \exp \left(\frac{-n t^{*}}{\theta}\right)}{x !} ; I_{\{0,1,2, \ldots\}}(x) \cdot \quad(3.2 .4 .3)
$$

Para encontrar-se o estimador de mäxima verossimilhança, deve-se obter a função de verossimilhança.

Dado que $\underline{r}$ falhas ocorrem, os tempos de falhas são distri buỉos condicionalmente, como uma variável ordenada uniformemente: 


$$
\begin{gathered}
f\left(t_{1, n}, t_{2, n}, \ldots, t_{r, n} \mid r\right)=\frac{r !}{\left(t^{*}\right)^{r}} ; \\
\quad 0<t_{1, n} \leqslant t_{2, n} \leqslant \ldots \leqslant t_{r, n}<t^{*}
\end{gathered}
$$

A função de verossimilhança ficarä:

$$
\begin{aligned}
L\left(t_{1, n}, t_{2, n}, \ldots,\right. & \left.t_{r, n} ; \theta\right)=f\left(t_{1, n}, t_{2, n}, \ldots, t_{r, n} \mid r\right) P(X=r) \\
= & \left(\frac{n}{\theta}\right)^{r} \exp \left(-\frac{n t^{*}}{\theta}\right) ; \\
& 0<t_{1, n} \leqslant t_{2, n} \leqslant \ldots \leqslant t_{r, n}<t^{*} . \quad(3.2 .4 . c)
\end{aligned}
$$

Tomando-se o logaritmo natural da expressão (3.2.4.c) derivando-se em relação a $\underline{\theta}$ e igualando-se a zero, obtëm-se o estimador de mäxima verossimilhança, que $\vec{e}$ dado por:

$$
\hat{\theta}=\frac{n t^{*}}{r} ; r>0
$$

Pode-se obter um intervalo de confiança aproximado para $\theta$

os limites do intervalo de confiança são determinados, re solvendo-se para $\underline{\lambda}$ as equações:

e

$$
\sum_{x=0}^{r} \frac{\lambda_{s}^{x} \exp \left(-\lambda_{s}\right)}{x !}=\frac{\alpha}{2},
$$

$$
\sum_{x=0}^{r-1} \frac{\lambda_{I}^{x} \exp \left(-\lambda_{I}\right)}{x !}=1-\frac{\alpha}{2}
$$

onde $\lambda=\frac{n t^{*}}{\theta}$. 
Em (3.2.4.e), $\frac{\alpha}{2}$ representa a probabilidade de observar $\underline{r}$ ou menos falhas para um dado $\underline{\lambda}$. Essa probabilidade decresce, à medida que $\underline{\lambda}$ cresce para um dado $\underline{r}$. Dessa forma, essa equação darä o limite superior para $\underline{\lambda}$. O conträrio é verdade para (3.2.4.f).

Pode-se assim, reescrever (3.2.4.e) e (3.2.4.f), como:

$$
\begin{aligned}
& P\left(X \leqslant r \mid \lambda_{S}\right)=\frac{\alpha}{2}, \\
& P\left(X<r \mid \lambda_{I}\right)=1-\frac{\alpha}{2} .
\end{aligned}
$$

Considerando-se a função gama incompleta:

$$
\Gamma(t ; \delta, \beta)=\frac{1}{\delta ! \beta^{\delta+1}} \int_{0}^{t} x^{\delta} \exp \left(-\frac{x}{\beta}\right) d x .
$$

Integrando $(3.2 .4 . h)$ sucessivamente por partes, obtēm-se um modelo que fa cilmente se reconhece como:

$$
\Gamma(y ; \delta, \beta)=1-\sum_{k=0}^{\delta} \frac{y^{k} \exp \left(-\frac{y}{\beta}\right)}{k ! \beta^{k}} .
$$

Com o intuito de combinar isto com a desejada soma de Poisson, fazendo-se $y=\lambda, \beta=1$ e $\delta=r$, e então (3.2.4.i), torna-se:

$$
\Gamma(\lambda ; r, 1)=1-P(R \leqslant r \mid \lambda),
$$

ou seja,

$$
\begin{aligned}
& \Gamma\left(\lambda_{I} ; r-1, I\right)=\frac{\alpha}{2}, \\
& \Gamma\left(\lambda_{S} ; r ; 1\right)=1-\frac{\alpha}{2} .
\end{aligned}
$$


Estas expressöes podem ser usadas para ächar $\lambda_{I}$ e $\lambda_{S}$ atravês de tabelas da distribuição gama incompleta. Contudo, a distribuição gama e a distribuição de qui-quadrado estão relacionadas.

Substituindo-se em $(3.2 .4 . h) \quad t=\lambda, \delta=r$ e $\beta=1$ terse-ä:

$$
\begin{aligned}
& \Gamma(\lambda ; r, 1)=\frac{1}{r !} \int_{0}^{\lambda} x^{r} \exp (-x) d x . \\
& \text { Fazendo-se } x=\frac{z}{2} \text { e } \quad r=\frac{v}{2}-1 \text { e, então, } d x=\frac{d z}{2} .
\end{aligned}
$$

Substituindo-se essas expressões em $(3.2 .4 \cdot k)$ ter-se-ä:

$$
\Gamma(\lambda ; r, I)=\frac{1}{\Gamma\left(\frac{\nu}{2}\right) 2^{\nu / 2}} \int_{0}^{2 \lambda} z^{\frac{\nu}{2}-1} \exp \left(-\frac{-z}{2}\right) d z \cdot \quad(3.2 .4 .2)
$$

Esta expressão possui distribuição de qui-quadrado com nụ mero de graus de liberdade igual a $\nu=2 r+2$.

Assim, o limite superior do intervalo de confiança é:

$$
2 \lambda_{s}=x_{2 r+2, \alpha / 2}^{2}
$$

o limite inferior do intervalo de confiança encontra-se de forma semelhante:

$$
2 \lambda_{I}=x_{2 r, 1-\alpha / 2}^{2}
$$

De $(3 \cdot 2.4 . m)$ e $(3 \cdot 2.4 \cdot n)$, podem-se obter os limites do in tervalo de confiança:

$$
P\left(\frac{x_{2 r, 1-\alpha / 2}^{2}}{2} \leqslant \lambda \leqslant \frac{x_{2 r+2, \alpha / 2}^{2}}{2}\right)=1-\alpha
$$


Como $\lambda=\frac{n t^{*}}{\theta}$, então:

$$
P\left(\frac{2 n t^{*}}{x_{2 r+2, \alpha / 2}^{2}} \leqslant \theta \leqslant \frac{2 n t^{*}}{x_{2 r, 1-\alpha / 2}^{2}}\right)=1-\alpha \text {, }
$$

ou

$$
\mathrm{P}\left(\frac{2 r \hat{\theta}}{x_{2 r+2, \alpha / 2}^{2}} \leqslant \theta \leqslant \frac{2 r \hat{\theta}}{x_{2 r, 1-\alpha / 2}^{2}}\right)=1-\alpha .
$$

Ainda que não sejam observadas falhas, pode-se calcular um limite de confiança inferior.

CoX (1953) discute o intervalo de confiança assim:

$$
\left(\frac{2 r \hat{\theta}}{x_{2 r+1, \alpha / 2}^{2}}, \frac{2 r \hat{\theta}}{x_{2 r, 1-\alpha / 2}^{2}}\right),
$$

e afirma que è ligeiramente menor que o dado em (3.2.4.p). Porēm, em alguns casos, possui nỉvel de confiança menor que $1-\alpha$.

3.3 Estimação da Função de Confiabilidade para a Distribuição Exponencial Monoparamétrica

A função de confiabilidade para a distribuição exponencial è estimada por:

$$
\hat{c}(t ; \theta)=\exp \left(\frac{-t}{\hat{\theta}}\right)
$$

o estimador de $\hat{\theta} \bar{e}$ dado por $(3.2 .1 . f)$ e $(3.2 .2 . b)$ para a censura do tipo II, sem reposição e com reposição, respectivamente. 
o estimador de $\dot{C}(t ; \theta)$ de $(3.3 . a)$ não $\vec{e}$ imparcial e isto é vālido para qualquer tipo de censura.

PUGH (1963) e BASU (1964) fornecem um estimador imparcial de mỉnima variância para $C(t ; \theta)$ quando a censura é do tipo II:

$$
\hat{C}(t ; \theta)=\left(1-\frac{t}{r \theta}\right)^{r-1} ; \quad r>1 .
$$

ZACKS e EVEN (1966) verificam que se $\frac{t}{r \hat{\theta}} \vec{e}$ pröximo de zero, então o estimador de mäxima verossimilhança possui menor erro quadrātico comparado com o estimador de minima variância.

0 viēs não terä muita importância em termos präticos, exceto quando as estimativas de $C(t ; \theta)$ são multiplicadas para determinar a confiabilidade do sistema.

O intervalo de confiança para $C(t ; \theta)$ é dado por:

$P\left[\exp \left(\frac{-t}{\theta_{I}}\right) \leqslant C(t ; \theta) \leqslant \exp \left(\frac{-t}{\theta_{S}}\right)\right]=1-\alpha$.

Supondo-se censura tipo II, (3.3.c) ficará:

$P\left[\exp \left(\frac{-t x_{2 r}^{2}, \alpha / 2}{2 r \hat{\theta}}\right) \leqslant c(t ; \theta) \leqslant \exp \left(\frac{-t x_{2 r, 1-\alpha / 2}^{2}}{2 r \hat{\theta}}\right)\right]=1-\alpha$.

Se a censura for do tipo I, e o teste for com reposição

(3.3.c), ficarā:

$P\left[\exp \left(\frac{-t x_{2 r+2, \alpha / 2}^{2}}{2 r \hat{\theta}}\right) \leqslant c(t ; \theta) \leqslant \exp \left(\frac{-t x_{2 r, 1-\alpha / 2}^{2}}{2 r \hat{\theta}}\right)\right]=1-\alpha$ 
Em algumas situações, pode-se estar interessado no tempo que uma certa proporção da população sobrevive. Seja p a proporção que fa Tha at $\vec{e} o$ instante $t_{p}$, daí:

$$
C\left(t_{p} ; \theta\right)=1-p,
$$

mas,

$$
c\left(t_{p} ; \theta\right)=\exp \left(\frac{-t_{p}}{\theta}\right) .
$$

De (3.3.f) e (3.3.g), tem-se:

$$
t_{p}=\theta \ln \left(\frac{1}{1-p}\right)
$$

Uma estimativa ề dada por:

$$
\hat{t}_{p}=\hat{\theta} \ln \left(\frac{1}{1-p}\right)
$$

3.4 Estimação para a Distribuição Exponencial Biparamêtrica

Em algumas situações, a distribuição exponencial biparamétrica $\vec{e}$ o modelo apropriado. Por exemplo, um periodo de garantia pode ser olhado como o período sem falhas do ponto de vista do cliente. Também, no estudo da fadiga de alguns metais, sabe-se que existe um período durante o qual não ocorrem falhas.

A função de densidade é dada em (3.1.a). A função de verossimilhança para censura tipo II, quando o teste ë sem reposição, ficarä: 


$$
\begin{aligned}
L\left(t_{1, n}, t_{2, n}, \ldots\right. & \left.t_{r, n} ; \theta, \delta\right)= \\
= & \frac{n !}{(n-r) !} \frac{1}{\theta^{r}} \exp \left\{\frac{-\left[\sum_{i=1}^{r}\left(t_{i, n}-\delta\right)+(n-r)\left(t_{r, n}-\delta\right)\right]}{\theta}\right\} . \\
& \delta \leqslant t_{1, n} \leqslant t_{2, n} \leqslant \ldots \leqslant t_{r, n}
\end{aligned}
$$

Tomando-se o logaritmo natural, (3.4.a) $\vec{e}$ igual a:

$$
=\ln \frac{n !}{(n-r) !}-r \ln \theta-\frac{\sum_{i=1}^{r}\left(t_{i, n}-\delta\right)+(n-r)\left(t_{r, n}-\delta\right)}{\theta} \cdot(3.4 . b)
$$

Derivando-se em relação a $\underline{\theta}$ e igualando-se a zero, temse:

$$
\hat{\theta}=\frac{\sum_{i=1}^{r}\left(t_{i, n}-\delta\right)+(n-r)\left(t_{r, n}-\delta\right)}{r} ; \quad r>0 \cdot(3.4 \cdot c)
$$

Observando-se que $\operatorname{lnL}\left(t_{1, n}, t_{2, n}, \ldots, t_{r, n} ; \theta, \delta\right) \vec{e}$ uma função monotônica crescente em $\delta$ e $\delta \leqslant t_{1, n}$, conclui-se que:

$$
\hat{\delta}=t_{1, n}
$$

Como $t_{1, n}$ é a menor estatîstica de ordem, em uma amostra de tamanho $\underline{n}$, a função de densidade de $T_{1, n}$ e:

$$
\begin{aligned}
g\left(t_{1, n} ; \theta, \delta\right) & =n\left[1-F\left(t_{1, n}\right)\right]^{n-1} f\left(t_{1, n}\right) . \\
& =\frac{n}{\theta} \exp \left[\frac{-n\left(t_{1, n}-\delta\right)}{\theta}\right] ; \quad I_{[\delta, \infty)}\left(t_{1, n}\right) .
\end{aligned}
$$


o valor esperado de $\mathrm{T}_{1, \mathrm{n}}$ é:

$$
E\left(T_{1, n}\right)=\delta+\frac{\theta}{n}
$$

Do numerador de $(3.4 . c)$, substituindo $\delta$ por $t_{1, n}$, temse:

$$
V=\sum_{i=2}^{r}\left(t_{i, n}-t_{1, n}\right)+(n-r)\left(t_{r, n}-t_{1, n}\right)
$$

Utilizando-se um procedimento semelhante ao caso de vida minima zero, pode-se definir:

$$
T\left(t_{i, n^{-t}} i-1, n\right)=w_{i, n}=(n-i+1)\left(t_{i, n}-t_{i-1, n}\right) ; t_{0}=\delta \cdot \quad(3 \cdot 4 \cdot n)
$$

Então (3.4.b), pode ser reescrita como:

$$
\begin{gathered}
L\left(w_{1, n}, w_{2}, n, \ldots, w_{r, n} ; \theta\right)=\frac{1}{\theta^{r}} \exp \left(\frac{-\sum^{r} w_{i}, n}{\theta}\right) \\
0 \text { jac obiano dessa transformaçäo serä igual a: } \\
\frac{(n-r) !}{r !} .
\end{gathered}
$$

A distribuição marginal para $i=1,2, \ldots, r$ :

$$
g\left(w_{i, n} ; \theta\right)=\frac{1}{\theta} \exp \left(\frac{-w_{i, n}}{\theta}\right) ; \quad I_{[0, \infty)}\left(w_{i}\right), \theta>0, \quad(3.4 \cdot j)
$$

A expressão (3.4.g), devido a $(3.4 . h)$, torna-se:

$$
V=\sum_{i=1}^{r} w_{i, n}
$$


onde $2 v / \theta$ possui distribuição de qui-quadrado com 2 r-2 graus de Iiberdade. Portanto,

$$
E\left(\frac{2 V}{\theta}\right)=2 r-2
$$

ou

$$
E(V)=\theta(r-1)
$$

Os estimadores imparciais para $\underline{\theta}$ e $\delta$ são:

$$
\begin{aligned}
& \hat{\theta}=\frac{\sum_{i=2}^{r}\left(t_{i, n}-t_{1, n}\right)+(n-r)\left(t_{r, n}-t_{1, n}\right)}{r-1} ; r>1 \\
& \tilde{\delta}=t_{1, n}-\frac{\hat{\theta}}{n} .
\end{aligned}
$$

A função de confiabilidade è estimada por:

$$
\hat{C}(t ; \tilde{\theta}, \tilde{\delta})=\exp \left[\frac{-(t-\tilde{\delta})}{\tilde{\theta}}\right] \quad I_{[\tilde{\delta}, \infty)}(t) .
$$

Para determinar o intervalo de confiança para $\delta$, devese usar o fato de que $\frac{2 n\left(T_{1, n}-\delta\right)}{\theta}$ e $\frac{2(r-1) \tilde{\theta}}{\theta}$ são independentes e tendo, ainda, cada uma distribuição de qui-quadrado com 2 e $2(r-1)$ graus de 1iberdade respectivamente. A razão $w=\frac{n\left(T_{1}-\delta\right)}{\tilde{\theta}}$ possui distribuição $F$ com 2 e $2(r-1)$ graus de liberdade respectivamente. 0 limite de confiança inferior para a vida mỉnima é:

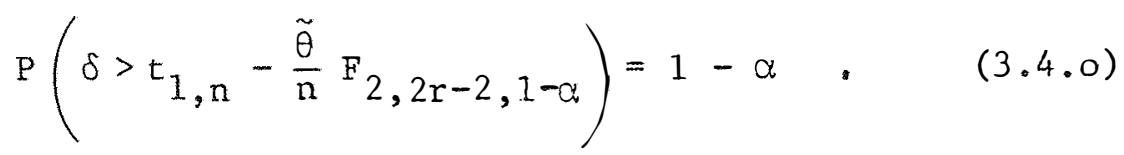

Um limite de confiança superior para a vida mỉnima ê dado pela menor esta tística de ordem, isto $\bar{e}, t_{1, n}$. 
Como $\frac{2(r-1) \tilde{\theta}}{\theta}$ apresenta distribuição de qui-quadrado com $2(r-1)$ graus de liberdade, o intervalo de confiança ficarā:

$$
\mathrm{P}\left[\frac{2(r-1) \tilde{\theta}}{x_{2 r-2, \alpha / 2}^{2}} \leqslant \theta \leqslant \frac{2(r-1) \tilde{\theta}}{x_{2 r-2,1-\alpha / 2}^{2}}\right]=1-\alpha \cdot \quad(3.4 \cdot p)
$$

Para a censura tipo II, com reposição a função de verossimilhança ficarä:

$$
\begin{gathered}
L\left(t_{1, n}, t_{2, n}, \ldots, t_{r, n} ; \theta, \delta\right)=\left(\frac{n}{\theta}\right)^{r} \exp \left[\frac{-n\left(t_{r, n}-t_{1, n}\right)}{\theta}\right], \\
\delta \leqslant t_{1, n} \leqslant t_{2, n} \leqslant \ldots \leqslant t_{r, n}
\end{gathered}
$$

Para obter-se os estimadores de mäxima verossimilhança, deve-se tomar o logaritmo natural da expressão (3.4.q), derivar em relação $\underline{\delta}$ e $\underline{\theta}$ e igualar a zero. Assim, tem-se:

$$
\hat{\delta}=t_{1, n} \quad \text { e } \quad \hat{\theta}=\frac{n\left(t_{r, n}-t_{1, n}\right)}{r} ; r>0 \text {. }
$$

os estimadores imparciais e de mínima variância são:

$$
\tilde{\theta}=\frac{n\left(t_{r, n}-t_{1, n}\right)}{r-1} ; \quad r>1 \quad \text { e } \tilde{\delta}=\hat{\delta}-\frac{\tilde{\theta}}{n} \text {. }
$$

Analogamente ao caso sem reposição, pode-se obter o intervalo de confiança para $\delta$ e para $\underline{\theta}$.

Se $T_{i, n} ; i=1,2, \ldots, r$ são as $\underline{r}$ primeiras estatísticas de ordem de uma amostra de tamanho $\underline{n}$, proveniente de uma população com dis tribuição exponencial biparamétrica, então uma translação do tipo 
$\mathrm{T}_{i, \mathrm{n}-1}=\mathrm{T}_{\mathrm{i}+1, \mathrm{n}}-\mathrm{T}_{1, \mathrm{n}} ; \mathrm{i}=1,2, \ldots, \mathrm{r}-1$, resultâ em $\mathrm{r}-1$ estatísticas de ordem de uma amostra de tamanho $n-1$. 0 parâmetro $\underline{f}$ desconhecicio é elimina do e as estatỉsticas de ordem transladadas possuem distribuição exponencial monoparamētrica.

Os resultados discutidos em 3.2 .1 e 3.2 .2 podem ser apli cados a $T_{i, n-1} \quad i=1,2, \ldots, r-1 ;$ desde que $\underline{n}$ e $\underline{r}$ sejam substituidos por $n-1$ e $r-1$ respectivamente.

Para a censura tipo I, sem reposição, a função de verossí milhança ficarä:

$$
\begin{aligned}
L\left(t_{1, n}, t_{2, n}, \cdots,\right. & \left.t_{r, n} ; \theta, \delta\right)= \\
= & \frac{n !}{(n-r) ! \theta^{r}} \exp \left\{-\left[\sum_{i=1}^{r}\left(t_{i, n}-\delta\right)+(n-r)\left(t^{*}-\delta\right)\right]\right. \\
& \delta<t_{1, n} \leqslant t_{2, n} \leqslant \cdots \leqslant t_{r, n}<t^{*} .
\end{aligned}
$$

Para obter-se os estimadores de mäxima verossimilhança, deve-se tomar o logaritmo natural da expressão $(3.4 . t)$, derivar em rela-ção a $\underline{\delta}$ e $\underline{\theta}$ e igualar a zero. Assim tem-se:

$$
\hat{\delta}=t_{1, n} \text { e } \hat{\theta}=\frac{\sum_{i=2}^{r}\left(t_{i, n}-t_{1, n}\right)+(n-r)\left(t^{*}-t_{1, n}\right)}{r} \quad r>0 \cdot(3 \cdot 4 \cdot u)
$$

Como as estatisticas de ordem transladadas

$$
\mathrm{T}_{\mathrm{i}, \mathrm{n}-1}=\mathrm{T}_{\mathrm{i}+1, \mathrm{n}}-\mathrm{T}_{1, \mathrm{n}} ; \mathrm{i}=1,2, \ldots, \mathrm{r}-1,
$$

possuem distribuição exponencial monoparamêtrica, pode-se utilizar os resultados discutidos em 3.2 .3 , desde que $\underline{r}, \underline{t}^{*}$ e $\underline{r}_{\underline{m}}$ sejam substituidos 
por $r-1, t^{*}-t_{1, n}$ e $n-1$ respectivamente.

Quando o teste for com reposição, a função de verossimi-

Thança ficarä:

$$
\begin{gathered}
L\left(t_{1, n}, t_{2, n}, \ldots, t_{r, n} ; \theta, \delta\right)=\left(\frac{n}{\theta^{-}}\right)^{r} \exp \left[\frac{-n\left(t^{*}-\delta\right)}{\theta}\right] \\
\delta<t_{1, n} \leqslant \ldots \leqslant t_{r, n}<t^{*} .
\end{gathered}
$$

Tomando-se o logaritmo natural da expressão $(3.4 . v)$, derí vando-se em relação a $\delta$ e $\underline{\theta}$, igualando-se a zero, obtêrn-se os estimadores de māxima verossimahança para $\delta$ e $\theta$ que são respectivamente:

$$
\begin{aligned}
& \hat{\delta}=t_{1, n} \text { e } \hat{\theta}=\frac{n\left(t^{*}-t_{1, n}\right)}{r} ; r>0 \quad(3.4 \cdot x) \\
& \text { Considerando-se novamente as estatisticas de ordem trans- }
\end{aligned}
$$
ladadas, podem-se utilizar os resultados discutidos em 3.2 .4 , desde que $\underline{r}$ e $\underline{t}^{*}$ sejam substituidos por $r-1$ e $t^{*}-t, n^{*}$

\section{5 - Testes de Hipöteses}

Na ärea da inferência estatỉstica, denominada Testes de Hipóteses, usar-se-âa o sỉmbolo $\mathrm{H}_{\mathrm{o}}$ para indicar a hipótese de nulidade e o simbolo $\mathrm{H}_{1}$ para indicar a hipōtese alternativa. Seja $\left(t_{1}, t_{2}, \ldots, t_{n}\right)$ uma amostra aleatöria, retirada da população, com função de densidade $f(t ; \theta)$; $\theta \in \Omega, \Omega$ é denominado espaço do parâmetro. Pode-se, assim, formular uma hi pótese estatística de que o verdadeiro valor do parâmetro $\underline{\theta}$ pertence a 
um subconjunto do espaço paramétrico $\Omega_{0}$ versus a hipötese alternativa de que $\theta$ pertence a $\Omega_{1}$. Geralmente $\Omega_{0} \cup \Omega_{I}=\Omega ; \Omega_{0} \varepsilon \Omega_{1}$ são aisjuntos, mas não è necessārio que sejam complementares. A hipótese è denominada simples, se especifica completamente a distribuição; serä composta, se não especificä-la completamente.

Em Teste de Hipótese, podem-se cometer dois tipos de erros. O erro tipo I ocorre, quando se rejeita $H_{0}$ e $H_{0} \bar{e}$ verdadeira. 0 erro tipo II ocorre, quando se aceita $\mathrm{H}_{\mathrm{O}}$ e $\mathrm{H}_{\mathrm{o}} \overline{\mathrm{e}}$ falsa.

0 esquema bäsico de Teste de Hipötese ê retirar uma amostra aleatöria $\left(t_{1}, t_{2}, \ldots, t_{n}\right)$ da população em que se estä interessado e calcular a estatỉstica teste:

$$
T=T\left(t_{1}, t_{2}, \ldots, t_{n}\right)
$$

Se a estatỉstica $\mathrm{T}$ cair em alguma região crîtica $\mathrm{C}$ prë-determinada, rejei ta-se a hipótese $\mathrm{H}_{\mathrm{O}}$.

A função poder do teste, representada por $B(\theta) \bar{e}:$

$$
\beta(\theta)=P\left(\text { rejeitar } H_{0} \mid \theta\right)=P(T \in C) \text {. }
$$

A probabilidade do erro tipo I è dada por:

$$
\alpha=\mathrm{P}\left(\mathrm{H}_{1} \mid \mathrm{H}_{0}\right)=\beta(\theta) ; \quad \theta=\theta_{0},
$$

e a probabilidade do erro tipo II ê dada por:

$$
\beta=P\left(H_{0} \mid H_{1}\right)=1-\beta(\theta) ; \quad \theta=\theta_{1} .
$$


0 tamanho do teste (ou da região crítica) é o

$$
\sup _{\theta} \in_{\Omega_{0}} \beta(\theta) .
$$

Muitos estatísticos usam o termo nỉvel de significância.

Quando a hipótese de nulidade, bem como a hipótese alternativa são simples, o Lema de Neyman-Pearson dâ-nos um método de encontrar a melhor região crítica de tamanho $\alpha$.

Se existe uma região crỉtica $C$, de tamanho $\alpha$ e uma constante $\underline{k}$, tal que:

$$
\frac{\prod_{i=1}^{n} f\left(t_{i} ; \theta_{1}\right)}{\prod_{i=1}^{n} f\left(t_{i} ; \theta_{0}\right)} \geqslant k, \text { para todos os pontos de } c \text {, }
$$

e

$$
\frac{\prod_{i=1}^{n} f\left(t_{i} ; \theta_{1}\right)}{\prod_{i=1}^{n} f\left(t_{i} ; \theta_{0}\right)}<k, \quad \begin{aligned}
& \text { para todos os pontos que não perten- } \\
& \text { cem a } C,
\end{aligned}
$$

então $C$ é a região crîtica mais poderosa de tamanho $\alpha$, tem-se então, um teste mais poderoso.

Entretanto, em muitos problemas, a hipötese $H_{0}$ ou $H_{1} \quad \bar{e}$ composta; isto ê, $H_{0}: \theta \in \Omega_{0}$ versus a hipötese alternativa $H_{1}: \theta \in \Omega-\Omega_{0}$. A região crítica $C$, tem tamanho menor ou igual a $\alpha$ para todo $\theta \in \Omega_{0}$.Tal região crítica pode ser a mais poderosa para algum $\underline{\theta}$, na alternativa $\Omega-\Omega_{\mathrm{o}}$, e pode não ser mais poderosa para outros valores. Se C $\vec{e}$ a mais poderosa para todo $\theta \in \Omega-\Omega_{o}$, então $C$ é denominada a região crítica uniformemente mais poderosa. 
$\widehat{E}$ possivel determinar um teste uniformemente mais poderoso, quando os testes são do tipo $H_{0}: \theta \leqslant \theta_{0}$ versus $H_{1}: \theta>\theta_{0}$ ou $H_{0}: \theta>0$ versus $\mathrm{H}_{1}: \theta<\theta_{0}$.

Para encontrar as regiöes crïticas, considerain-se que as variáveis aleatörias $\mathrm{T}_{1}, \mathrm{~T}_{2}, \ldots, \mathrm{I}_{\mathrm{n}}$ são independentes e identicanente distribuỉdas com função densidade, $f(t ; \theta)$. Supondo-se que $f\left(t_{1}, t_{2}, \ldots ; \theta\right)$ pode ser escrita como:

$f\left(t_{1}, t_{2}, \ldots ; \theta\right)=A(\theta) \exp \left[Q(\theta) T\left(t_{1}, t_{2}, \ldots, t_{n}\right)\right], h\left(t_{1}, t_{2}, \ldots, t_{n}\right)$

onde $Q(\theta)$ se supõe que seja uma função monotōnica estritamentedecrescente e $\theta \in \Omega$. A classe de densidade que satisfaz às condiçôes de (3.5.€) e denominada "famỉlia" exponencial de densidades.

Quando $\mathrm{H}_{\mathrm{c}}: \theta \leqslant \theta_{0}$ versus $\mathrm{H}_{1}: \theta>\theta_{0}$, um teste, uniformemente mais poderoso de tamanho $\alpha$, é rejeitar $\mathrm{H}_{0}$, se $\mathrm{T}\left(\mathrm{t}_{1}, \mathrm{t}_{2}, \ldots, \mathrm{t}_{\mathrm{n}}\right)>\mathrm{C}$, onde $c \bar{e}$ tal que $P\left[T\left(t_{1}, t_{2}, \ldots, t_{n}\right)>C\right]=\alpha$. Quando $H_{0}: \theta \geqslant \theta_{0}$ versus $\mathrm{H}_{1}: \theta<\theta_{0}$, um teste uniformemente mais poderoso de tamanho $\alpha$ é rejeitar $H_{0}$, se $T\left(t_{1}, \ldots, t_{n}\right)<c$, onde: $P\left[T\left(t_{1}, t_{2}, \ldots, t_{n}\right)<c\right]=\alpha$.

Quando se quer testar $H_{0}: \theta=\theta_{0}$ versus $H_{1}: \theta \neq \theta_{0}$, um teste mais poderoso imparcial de tamanho $\alpha$, ê rejeitar $\mathrm{H}_{\mathrm{o}}$ se $T\left(t_{1}, t_{2}, \ldots, t_{n}\right)<C_{1}$ ou $T\left(t_{1}, t_{2}, \ldots, t_{n}\right)>C_{2}$, onde $C_{1}$ e $C_{2}$ são tais que $P\left[C_{1} \leqslant T\left(t_{1}, t_{2}, \ldots, t_{n}\right) \leqslant C_{2}\right]=1-\alpha_{\text {。 }}$ 


\subsubsection{Censura do Tipo II}

A fim de determinar a região crïtica, para o teste $\mathrm{H}_{0}: \theta=\theta_{0}$ versus $\mathrm{H}_{1}: \theta=\theta_{1}\left(\theta_{1}<\theta_{0}\right)$ utiliza-se a expressão que foi dada por (3.5.d), tem-se entäo:

$$
\frac{L_{1}}{L_{0}}=\frac{L\left(t_{1, n}, t_{2, n}, \ldots, t_{r, n} ; \theta_{1}\right)}{L\left(t_{1, n}, t_{2, n}, \cdots, t_{r, n} ; \theta_{0}\right)}>k \quad, \quad \theta_{1}<\theta_{0}, \quad \text { (3.5.1.a) }
$$

Quando o teste for sem reposição, a função de verossimi-

lhança ficará:

$$
\begin{aligned}
L\left(t_{1, n}, t_{2, n}, \ldots, t_{r, n} ; \theta_{i}\right) & = \\
& =\frac{n !}{(n-r) !}\left(\frac{1}{\theta_{i}}\right)^{r} \exp \left(\frac{-\left[\sum_{i=1}^{r} t_{i}+(n-r) t_{r, n}\right]}{\theta_{i}}\right) .
\end{aligned}
$$

Substituindo (3.5.1.b) em (3.5.1.a), tem-se

$$
\frac{\mathrm{L}_{1}}{\mathrm{~L}_{0}}=\left(\frac{\theta_{0}}{\theta_{1}}\right)^{r} \exp \left\{-\left[\sum_{i=1}^{r} t_{i, n}+(n-r) t_{r, n}\right]\left(\frac{1}{\theta_{1}}-\frac{1}{\theta_{0}}\right)\right\}
$$

ou:

$$
\frac{\mathrm{L}_{1}}{\mathrm{~L}_{0}}=\left(\frac{\theta_{0}}{\theta_{1}}\right)^{r} \exp \left[-\mathrm{r} \hat{\theta}\left(\frac{1}{\theta_{1}}-\frac{1}{\theta_{0}}\right)\right]>\mathrm{k}
$$

onde $\hat{\theta}$ foi dado por $(3.2 .1 . e)$.

Como $\theta_{1}<\theta_{0}$, então $\frac{1}{\theta_{1}}-\frac{1}{\theta_{0}}>0$. 0 lado esquerdo da de sigualdade (3.5.1.c) decresce, quando $\hat{\theta}$ cresce, portanto a região de rejeição da hipötese $H_{0} \vec{e}$ da forma $\hat{\theta}<C$.

$$
\text { Para calcular } C \text {, deve-se lembrar que } \frac{2 r \hat{\theta}}{\theta} \text { possui distri- }
$$
buição qui-quadrado com $2 r$ graus de liberdade. Então, 


$$
\hat{\theta}=\theta \frac{x_{2 r}^{2}}{2 r} .
$$

Tem-se então, que:

$$
P\left(\hat{\theta} \geqslant C \mid \theta=\theta_{0}\right)=1-\alpha,
$$

ou:

$$
P\left(\frac{\theta}{0} \frac{x_{2 r}^{2}}{2 r} \geqslant c\right)=1-\alpha,
$$

do que resulta:

$$
P\left(X_{2 r}^{2} \geqslant \frac{2 r C}{\theta_{0}}\right)=1-\alpha .
$$

Portanto: $\frac{2 r c}{\theta_{0}}=\chi_{2 r, 1-\alpha}^{2}$, então:

$$
c=\frac{\theta_{0} x_{2 r ; 1-\alpha}^{2}}{2 r} .
$$

A tabela 1 do Apêndice fornece os valores $c / \theta_{0}$ para fazer o teste $H_{0}: \theta \geqslant \theta_{0}$ versus $H_{1}: \theta<\theta_{0}$. Por exemplo se $\theta_{0}=1000 ; \quad \alpha=0,05$ e $r=6$, a região de rejeição da hipötese $H_{0} \vec{e} \hat{\theta}<\theta_{0}\left(\frac{C}{\theta_{0}}\right)$. Da tabela 1 do Apêndice tem-se, para estes dados, que $\frac{C}{\theta_{0}}=0,436$; como $\theta_{0}=1000$ a hipötese $H_{0}$ é rejeitada, se $\hat{\theta}<1000$. 0,436 ou $\hat{\theta}<436$ ao nivel de sig nificância $\alpha=0,05$.

A região de aceitação da hipötese $H_{0}$ é:

$$
\hat{\theta} \geqslant \frac{\theta_{0} x_{2 r, 1-\alpha}^{2}}{2 r}
$$

A quantidade $\mathrm{P}_{\mathrm{a}}(\theta)$ que $\overrightarrow{\mathrm{e}} I-\beta(\theta)$ pode ser obtida:

$$
P_{a}(\theta)=P\left(\hat{\theta} \geqslant \frac{\theta_{0} x_{2 r, 1-\alpha}^{2}}{2 r}, \theta\right) .
$$


Daì, ter-se-à:

$\mathrm{P}_{\mathbf{a}}(\theta)=\mathrm{P}\left(\frac{2 \mathrm{r} \hat{\theta}}{\theta} \geqslant \frac{\theta_{0}}{\theta} \quad x_{2 \mathrm{r}, 1-\alpha}^{2} \mid \theta\right)=P\left(x_{2 \mathrm{r}}^{2} \geqslant \frac{\theta_{0}}{\theta} \quad x_{2 \mathrm{r}, 1-\alpha}^{2} \mid \theta\right) .(3.5 .1 . j)$

Esta probabilidade ẻ função do parâmetro $\underline{\theta}$ e do nümero de falhas $\underline{r}$, podendo assim ser calculada para diferentes valores de $\underline{\theta}$ e $\underline{r}$. Representan do-se estes resultados em um gräfico, obtëm-se uma curva que è denominada curva caracteristica de operação.

Atravës das curvas caracterïsticas, pode-se calcular a probabilidade de aceitar a hipötese $H_{0}$ para valores pre-fixadosde $\theta_{0}$ e $\theta_{1}$. Tais curvas, para esta situação, serão fornecidas no A pêndice.

Quando o teste ê formulado como $\mathrm{H}_{0}: \theta \leqslant \theta_{0}$ e $\mathrm{H}_{1}: \theta>\theta_{0}$, a um nível de significância $\alpha$, a probabilidade de aceitar a hipötese $H_{0}$ é:

$$
p\left(\frac{2 r \hat{\theta}}{\theta} \leqslant x_{2 r, \alpha}^{2} \mid \theta=\theta_{0}\right)=1-\alpha .
$$

A região de aceitação ë:

$$
\hat{\theta} \leqslant \frac{\theta_{0} x_{2 r, \alpha}^{2}}{2 r},
$$

e a de rejeição è $\hat{\theta}>C$, onde. $c \vec{e}$ :

$$
c=\frac{\theta_{0} \quad x_{2 r, \alpha}^{2}}{2 r}
$$

A tabela 2 do Apêndice fornece os valores $\frac{\mathrm{C}}{\theta_{0}}$ para fazer o teste $H_{0}: \theta \leqslant \theta_{0}$ versus $H_{1}: \theta>\theta_{1}$ por exemplo, se $\theta_{0}=500$, $r=5$ e $\alpha=0,10$; a região de rejeição da hipötese $H_{0}$ é dada por:

$$
\hat{\theta}>\theta_{0}\left(\frac{C}{\theta_{0}}\right) \text {. }
$$


Da tabela 2 tem-se, para estes dados, que $\frac{C}{\theta_{0}}=1,599$; como $\theta_{0}=500$, a hipótese $H_{0}$ será rejeitada, se $\hat{\theta}>799,5$ ao nível de significârcia $\alpha=0,10$.

A curva caracterîstica de operação para o teste $\ddot{\theta}$ obtida de:

$$
P_{a}(\theta)=P\left(x_{2 r}^{2} \leqslant \frac{\theta_{0}}{\theta} \quad x_{2 r, \alpha}^{2} \mid \theta\right) .
$$

Algumas curvas para essa situação seräo fornecidas

Apêndice.

Pode-se verificar de $(3,5.1 . \hat{i})$ e de $(3,5.1 . \mathrm{m})$ que a curva característica de operação depende somente de $\underline{r}$ e não de $\underline{n}$.

Para um conjunto fixado $\left(\theta_{0}, \theta_{1}, \alpha, \beta\right)$ pode-se calculax $\underline{r}$, digamos $r_{0}$ e $C$. Por exemplo, para o teste $H_{0}: \theta \geqslant \theta_{0}$ versus $H_{1}: \theta<\theta_{1}$, procura-se o menor valor de $\underline{r}$, tal que:

$$
\begin{aligned}
& P\left(\hat{\theta} \geqslant c \mid \theta=\theta_{0}\right)=1-\alpha, \\
& P\left(\hat{\theta} \geqslant c \mid \theta=\theta_{1}\right)=\beta .
\end{aligned}
$$

De $(3 \cdot 5 \cdot 1 . j)$ tem-se:

$$
\frac{x_{2 r, 1-\alpha}^{2}}{x_{2 r, \beta}^{2}}=\frac{\theta_{1}}{\theta_{0}}
$$

e deve-se obter o menor $\underline{r}$, tal que:

$$
\frac{x_{2 r, 1-\alpha}^{2}}{x_{2 r, \beta}^{2}} \geqslant \frac{\theta_{1}}{\theta_{0}}
$$

A tabela 3 do Apêndice fornece 


$$
\frac{x_{2 r_{0}, 1-\alpha}^{2}}{x_{2 r_{0}, \beta}^{2},}
$$

para alguns valores de $\alpha$ e $\beta$. Atravēs da mesma pode-se determinar o nümero de falhas que se deve observar no teste. Por exemplo, se $\mathrm{H}_{\mathrm{o}}: \theta=1000$ e $\mathrm{H}_{1}: \theta=250$, adotando-se $\alpha=0,05$ e $\beta=0,10$, da tabela 3 , obtëmse $r=6$, pois $0,282 \geqslant \frac{250}{1000} ; 0,282 \geqslant 0,250$. Calcula-se $\beta^{\prime}$ de $(3.5 .1 . j)$, tomando-se $\theta=\theta_{1}$

$$
\beta^{\prime}=P_{a}\left(\theta_{1}\right)=P\left(x_{2 r}^{2} \geqslant \frac{\theta_{0}}{\theta_{1}} x_{2 r, 1-\alpha}^{2}\right)=P\left(x_{12}^{2} \geqslant 20,92\right) \cong 0,05
$$

podem-se verificar que $\alpha=0,05$, mas $\beta \leqslant 0,10$.

$$
\text { Para o teste } H_{0}: \theta \leqslant \theta_{0} \text { versus } H_{1}: \theta>\theta_{0} \text { pode-se tambēm }
$$
encontrar o menor valor de $\underline{r}$, tal que:

$$
\begin{aligned}
& P\left(\hat{\theta} \leqslant c \mid \theta=\theta_{0}\right)=\alpha, \\
& P\left(\hat{\theta} \leqslant c \mid \theta=\theta_{1}\right)=1-\beta .
\end{aligned}
$$

De $(3.5 .1 . n)$ tem-se:

$$
\frac{x_{2 r_{0}, \alpha}^{2}}{x_{2 r_{0}, 1-\beta}^{2}}=\frac{\theta_{1}}{\theta_{0}} .
$$

Deve-se procurar o valor de $\underline{r}$, tal que:

$$
\frac{x_{2 r_{0}, \alpha}^{2}}{x_{2 r_{0}, 1-\beta}^{2}} \geqslant \frac{\theta_{1}}{\theta_{0}}
$$

A tabela 4 do Apêndice fornece 


$$
\frac{x_{2 r_{0}, \alpha}^{2}}{x_{2 r_{0}, 1-\beta}^{2}}
$$

para alguns valores de $\alpha$ e $\beta$. Através desta tabela, pode-se determinar o menor valor de falhas que se deve observar no teste.

Em ambos os casos, selecionou-se o menor $r_{0}$ que forneça $\alpha$ exato, $\operatorname{com} \beta^{\prime} \leqslant \beta$.

Quando o teste é com reposição, o procedimento $\vec{e}$ anäjogo. O estimador de $\underline{\underline{e}}$ ebtido da expressão dada em (3.2.2.b).

GRUBBS (1973), para obter uma solução analítica de

$$
\frac{x_{2 r_{0}, \alpha}^{2}}{x_{2 r_{0}, 1-\beta}^{2}}=\frac{\theta_{1}}{\theta_{0}}
$$

aproxima a distribuição de qui-quadrado pela distribuição normal, tal como:

$$
\frac{\theta_{1}}{\theta_{0}}=\frac{v\left[1-\frac{2}{9 v}+z_{\alpha} \sqrt{\frac{2}{9 v}}\right]^{3}}{v\left[1-\frac{2}{9 v}+z_{1-\alpha} \sqrt{\frac{2}{9 v}}\right]^{3}} \text {. ond e } v=2 r
$$

$\operatorname{Se}\left(\theta_{0}, \theta_{1}, \alpha, \beta\right)$ são fixados, pode-se calcular $\underline{r}$. Tem-se, então:

$$
r=\frac{4(\delta-1)^{2}}{9\left[\delta z_{\alpha}-z_{1-\beta}+\sqrt{\left(\delta z_{\alpha}-z_{1-\beta}\right)^{2}+4(\delta-1)^{2}}\right]^{2}}=\frac{4}{9\left[\sqrt{\lambda^{2}+4}-\lambda\right]^{2}}
$$

onde

$$
\delta=\sqrt[3]{\frac{\theta_{0}}{\theta}} \quad \text { e } \quad \lambda=\frac{z_{1-\beta}-\delta z_{\alpha}}{\delta-1} \text {. }
$$


Na tabela 5, enumera-se as estatísticas usadas e as regiões crỉticas para os testes relativos à média quando a distribuição é exponencial monoparamétrica.

Para a distribuição exponencial biparamëtrica estes resultados podem ser aplicados desde que $\underline{n}$ e $\underline{r}$ sejam substituidos por $n-1$ e $\mathrm{r}-1$, respectivamente.

Como jā se mencionou, a curva característica de operação depende somente de $\underline{r}$ e não de $\underline{n}$. Isto significa que a mesma precisão é ob tida, sejam somente $\underline{r}$ unidades testadas até falharem ou sejam 프 unidades colocadas no teste, e o teste termina após a r-ésima falha.

Surge, neste ponto, uma questão lōgica: por que colocarse mais que $\underline{r}$ unidades no teste? A resposta estä na economia do tempo de teste.

Para analisar esta afirmaçäo, deve-se calcular o tempo mé dio esperado para a execução do teste, que é representado por $E\left(T_{r, n}\right)$. A expressão

$$
E\left(T_{r, n}\right)=\frac{\theta}{n}+\frac{\theta}{n-1}+\ldots+\frac{\theta}{n-r+1}=\theta \underset{i=1}{\sum_{i=1}} \frac{1}{n-i+1}
$$

fornece o tempo médio esperado.

$$
\text { Para se encontrar esta förmula, deve-se lembrar (3.2.1) }
$$

$\mathrm{T}_{\mathrm{r}, \mathrm{n}}=\sum_{i=1}^{\mathrm{r}} \mathrm{Y}_{\mathrm{i}, \mathrm{n}}$ onde $\mathrm{Y}_{\mathrm{i}}=\mathrm{T}_{\mathrm{i}, \mathrm{n}}{ }^{-\mathrm{T}_{\mathrm{i}-1, \mathrm{n}}} ; \quad \mathrm{i}=1,2, \ldots, \mathrm{r} ; \quad \mathrm{t}_{\mathrm{o}}=0$ 
As variáveis aleatōrias $Y_{i, n}$ são independentes $€$ cada $Y_{i, n}$ tem função de densidade dada por:

$$
f\left(y_{i, n} ; \theta\right)=\frac{(n-i+1)}{\theta} \exp \left[\frac{\left.-(n-i+1) y_{i, n}\right] ; \quad I_{[a, \infty)}\left(y_{i, n}\right) ; \quad \theta>0 ;}{(3.5 .1 .5)}\right.
$$

Pode-se calcular o valor esperado da variāvel aleatória. $Y_{i, n} ;$ assim tem-se:

$$
E\left(Y_{i, n}\right)=\frac{(n-i+1)}{\theta} \int_{0}^{\infty} y_{i, n} \exp \left[\frac{-(n-i+1) y_{i, n}}{\theta}\right] d y_{i, n}
$$

obtēm- -se

$$
E\left(Y_{i, n}\right)=\frac{\theta}{n-i+1}
$$

Assim o tempo médio esperado para a execução do teste é:

$$
E\left(T_{r, n}\right)=E\left(\sum_{i=1}^{r} Y_{i, n}\right)=\sum_{i=1}^{r} E\left(Y_{i, n}\right)=\theta \sum_{i=1}^{r} \frac{1}{n-i+1} .
$$

Pode-se, então, verificar que $E\left(T_{r, n}\right)$ decresce, quando $\underline{n}$ cresce.

A variância da expressão (3.5.1.r) è dada por:

$$
V\left(T_{r, n}\right)=\theta^{2} \sum_{i=1}^{r}\left(\frac{1}{n-i+1}\right)^{2}
$$

A tabela 6 do Apêndice fornece $\left(\frac{]_{0}}{\theta_{0}}\right) E\left(T_{r, n}\right)$ para värios valores de $\underline{r}$ e $\underline{n}$. 
Por exemplo, se $\mathrm{n}=20$ e $\mathrm{r}=10$, o tempo médio esperado para a execução do teste é $0,6688 \theta$.

A economia do tempo de duração do teste pode ser obtida, considerando-se a razâo

$$
E_{f}=\frac{E\left(T_{r, n}\right)}{E\left(T_{r, r}\right)} \text {, }
$$

ond e $\mathrm{E}\left(\mathrm{T}_{\mathrm{r}, \mathrm{r}}\right)$ é o tempo médio esperado do teste, quando $\mathrm{n}=\mathrm{r}$ unidades, ne le são colocadas, observando-se todas as falhas. Neste caso, tem-se

$$
E\left(T_{r, r}\right)=\sum_{i=1}^{r} \frac{1}{r-i+1}
$$

Pode-se calcular $E_{f}$ de $(3.5 .1 . v)$ e $(3.5 .1 . x)$, tendo-se

$$
E_{f}=\frac{E\left(T_{r, n}\right)}{E\left(T_{r, r}\right)}=\frac{\sum_{i=1}^{r} \frac{1}{n-i+1}}{\sum_{i=1}^{r} \frac{1}{r-i+1}} \text {. }
$$

A tabela 7 do Apêndice fornece os valores de $E_{f}$ para alguns valores de $r$ e $n$; por exemplo, se $n=20$ e $r=4$ e o teste é sem reposição, temse uma economia de tempo de 0,104, isto é, o teste terâa apenas $10 \%$ da duração daquele em que quatro unidades são colocadas no teste e todas são mantidas até falharem.

Por outro lado, quando a censura é do tipo II com reposi ção, o tempo médio esperado é dado por:

$$
E\left(T_{x, n}\right)=\frac{r \theta}{n} \text {, }
$$

vis to que, 


$$
\begin{aligned}
T_{r, n}=T_{1, n}+\left(T_{2, n}-T_{1, n}\right)+\ldots+\left(T_{r, n}-T_{r-1, n}\right) \\
E\left(T_{r, n}\right)=E\left(T_{1, n}\right)+E\left(T_{2, n}-T_{1, n}\right)+\ldots+E\left(T_{r, n}-T_{r-1, n}\right) \\
=\frac{\theta}{n}+\frac{\theta}{n}+\ldots+\frac{\theta}{n}=\frac{r \theta}{n} .
\end{aligned}
$$

Desta forma, a economia de tempo serā dada por:

$$
E_{f}=\frac{E\left(T_{r, n}\right)}{E\left(T_{r, r}\right)}=\frac{\frac{r \theta}{n}}{\theta}=\frac{r}{n} .
$$

\section{5 .2 - Censura do Tipo I}

No caso da censura do Tipo I, a duração do teste é especi ficada para algum tempo $\underline{t}^{*}$. Neste caso, o nümero de falhas é uma variävel aleatória e iremos supor, inicialmente, que a amostragem è sem reposi ção.

Um teste do tipo $H_{0}: \theta \geqslant \theta_{0}$ versus $H_{1}: \theta<\theta_{0} \widehat{e}$ equivalente a $H_{0}: p \leqslant p_{0}$ versus $H_{1}: p>p_{0}$, onde $\underline{p} \bar{e}$ a probabilidade de que uma unidade falhe no intervalo de $\left[0, t^{*}\right]$. A probabilidade $\underline{p}$ é dada por:

$$
p=1-\exp \left(\frac{-t^{*}}{\theta}\right)
$$

sendo $\underline{p}$ è o parâmetro de uma distribuição binomial.

Pode-se definir uma variävel aleatöria Y, que assume os va lores um ou zero, isto é, Y assume o valor um, se a unidade falhar até $\underline{t}^{*}$, e zero, se a unidade não falhar atê $t^{*}$. 
A fim de determinar a regiäo crïtica, utiliza-se a expressão dada em (3.5.d). Tem-se, então:

$$
\frac{L\left(y_{1, n}, y_{2, n}, \cdots, y_{r, n} ; p_{1}\right)}{L\left(y_{1, n}, y_{2, n}, \cdots, y_{r, n} ; p_{0}\right)}=\frac{p_{1}^{\sum y_{i}}\left(1-p_{1}\right)^{n-\Sigma y_{i}}}{p_{0}^{\sum y_{i}}\left(1-p_{0}\right)^{n-\sum y_{i}}} \geqslant k,
$$

como $\mathrm{p}_{0}<\mathrm{p}_{1} ; \frac{\mathrm{L}_{1}}{\mathrm{~L}_{0}} \geqslant k$ se $\mathrm{x}=\Sigma \mathrm{y}_{i} \geqslant C$.

$$
c=\frac{\log k-n \log \left(\frac{1-p_{1}}{1-p_{0}}\right)}{\log \frac{p_{1}\left(1-p_{0}\right)}{p_{0}\left(1-p_{1}\right)}}=r_{0},
$$

o valor de $\underline{p}$ é obtido de (3.5.2.a).

A região crïtica pode ser obtida, resolvendo-se a equação:

$$
1-P\left(x \leqslant r_{0}-1 \mid p_{0}=p_{0}\right)=1-\sum_{x=0}^{r_{0}-1}\left(\begin{array}{l}
n \\
x
\end{array}\right) p_{0}^{x}\left(1-p_{0}\right)^{n-x} \leqslant \alpha
$$

Se o nümero de falhas é menor que $r_{0}$, aceita-se a hipóte se $\mathrm{H}_{\mathrm{O}}$, caso contrário rejeita-se $\mathrm{H}_{0}$.

Analogamente, podem-se determinar as regiões críticas para outros tipos de testes. Na Tabela 8 do Apêndice fornecem-se as estatís ticas e as regiões críticas para os mesmos, quando a distribuição é exponencial monoparamêtrica. Para a distribuição exponencial biparamêtrica estes resultados podem ser aplicados, desde que $\mathrm{n}, \mathrm{r}$ e $\underline{t}^{*}$ sejam substituidos por $n-1, r_{0}-1$ e $t^{*}-t_{1, n}$, respectivamente.

Para se obter o nümero mêdio de unidades necessärias, a fim de se tomar uma decisão, deve-se calcular $E(X)$, donde: 


$$
E(X)-\sum_{x=0}^{n} x\left(\begin{array}{l}
n \\
x
\end{array}\right) p^{x}(1-p)^{n-x} ; \quad I_{\{0,1,2, \ldots, n\}}(x),
$$

ou:

$$
E(X)=\sum_{x=0}^{r_{0}-1} x\left(x_{x}^{n}\right) p^{x}(1-p)^{n-x}+\sum_{x=r_{0}}^{n} x\left(x_{x}^{n}\right) p^{x}(1-p)^{n-x}
$$

mas,

$$
x\left(\frac{n}{x}\right)=\frac{n !}{(n-x) !(x-1) !}=n\left(\begin{array}{l}
n-1 \\
x-1
\end{array}\right)
$$

Substituindo (3.5.2.f) em (3.5.2.e) ter-se-âa:

$$
\begin{aligned}
E(X)=n p & {\left[\begin{array}{c}
n-1 \\
0
\end{array}\right)(1-p)^{n-1}+\left(\begin{array}{c}
n-1 \\
1
\end{array}\right) p(1-p)^{n-2}+\cdots } \\
& \left.+\left(\begin{array}{l}
n-1 \\
r_{0}-2
\end{array}\right) p^{r}{ }^{-2}(1-p)^{n-r_{0}+1}\right]+r_{0}\left[1-\sum_{x=0}^{r_{0}-1}\left(\begin{array}{c}
n \\
x
\end{array}\right) p^{x}(1-p)^{n-x}\right],
\end{aligned}
$$

ou :

$E(X)=n p\left[\sum_{x=0}^{r_{0}-2}\left(\begin{array}{c}n-1 \\ x\end{array}\right) p^{x}(1-p)^{n-x}\right]+r_{0}\left[1-\sum_{x=0}^{r_{0}^{-1}}\left(\begin{array}{c}n \\ x\end{array}\right) p^{x}(1-p)^{n-x}\right]$.

A fim de se determinar o tempo médio esperado para tomar uma decisão, deve-se calcular:

$$
E\left(T_{r, n}\right)=\sum_{X=0}^{r_{0}} P(X=x) E\left(T_{x, n}\right)
$$

ond e

$$
P(X=x)=\left(\begin{array}{l}
n \\
x
\end{array}\right) p^{x}(1-p)^{n-x} ; \cdot \quad I_{\left\{0,1,2, \ldots, x_{0}\right\}}(x) .
$$


e

$$
E(T, x, n)=\theta \sum_{i=1}^{x} \frac{1}{n-i+1}
$$

Para analisar-se o caso de censura tipo I com reposição supõe-se o teste $H_{0}: \theta \geqslant \theta_{0}$ versus $H_{1}: \theta<\theta_{0}$. A região cr $\ddot{i} t i c a$ é, neste caso, obtida de forma anảloga a amostragem sem reposição.

$$
x=\Sigma y_{i} \geqslant r_{0}
$$

A curva caracteristica de operação para o teste è dada por:

$$
P_{a}(\theta)=P\left(X \leqslant r_{0}-1\right)=\sum_{x=0}^{r_{0}-1} P(X=x) .
$$

Como jâ foi visto em 3.2.4., o número de falhas possui distribuição de Poisson com parâmetro $n t * / \theta$.

Assim,

$$
P(X=x)=\frac{\left(\frac{n t^{*}}{\theta}\right)^{x} \exp \left(\frac{-n t^{*}}{\theta}\right)}{x !} ; \quad I_{\{0,1,2, \ldots\}}(x) .
$$

Então, (3.5.2.i) ficarâ:

$$
P_{a}(\theta)=P\left(X \leqslant r_{0}-1\right)=\sum_{x=0}^{r_{0}-1} \frac{\left(\frac{n t^{*}}{\theta}\right) \exp \left(-\frac{n t^{*}}{\theta}\right)}{x !} .
$$

Como jă foi visto em 3.1 a distribuição de Poisson pode ser relacionada com a distribuição de qui-quadrado; é possivel, então, obte 
rem-se as regiões crïticas para algumas hipöteses em função desta distribuição. A tabela 9 do Apêndice fornece as estatisticas e as regiōes criticas para alguns testes, quando a distribuição è exponencial monoparamētrica.

Para a distribuição exponencial biparamëtrica estes resultados podem ser aplicados desde que $\underline{t}^{*}$ e $r_{0}$ sejam substituidos por $t^{*}-t_{1, n}$ e $r_{0}-1$.

A Eim de determinar o nümero mëdio de tentativas para tomar uma decisão, deve-se calcular $E(X)$, donde:

$$
E(X)=\sum_{x=0}^{r_{0}^{-1}} x P(X=x)+r_{0}\left[1-\sum_{X=0}^{r_{0}^{-1}} P(X=x)\right] \cdot \quad(3 \cdot 5 \cdot 2 \cdot 2)
$$

Substituindo $(3.2 .5 . j)$ em $(3.5 .2 . l)$ ter-se-ä:

$E(X)=\frac{n t^{*}}{\theta} \sum_{x=0}^{r_{0}-2}\left[\frac{\left(\frac{n t^{*}}{\theta}\right)^{x} \exp \left(\frac{-n t^{*}}{\theta}\right)}{x !}\right]+r_{0}\left[1-\sum_{x=0}^{r_{0}-1} \frac{\left(\frac{n t^{*}}{\theta}\right)^{x} \exp \left(\frac{-n t^{*}}{\theta}\right)}{x !}\right]$.

Para obter-se o tempo mêdio esperado, a fim de tomar uma decisão, deve-se calcular:

$$
E\left(T_{r, n}\right)=\frac{\theta}{n} E(X)
$$

onde $E(X)$ è dado por $(3.5 .2 . l)$. 


\subsubsection{Tamanho da Amostra}

o custo esperado do experimento, quando a cersura $\vec{e}$ do ti po II è dado por:

$$
E(\text { custo })=c_{1}^{*} E\left(T_{r, n}\right)+c_{2}^{*} k,
$$

onde $c_{1}^{*}$ e o custo de observação por unidade de tempo, $c_{2}^{*} \bar{e}$ o custo para colocar uma unidade no experimento e $k \underline{e}$ o nümero de unidades colocadas no experimento.

Se a amostragem ê sem reposição (3.5.3.a) ficarä:

$$
E \text { (custo) } n=c_{1}^{*} \theta_{0} \sum_{i=1}^{r} \frac{1}{n-i+1}+c_{2}^{*} n
$$

Pode-se calcular o tamanho da amostra, fixando $c_{1}^{*}, c_{2}^{*}, \underline{r}$ e variando n. O tamanho da amostra ótimo é aquele que minimiza o custo esperado do experimento,

De modo anälogo, pode-se obter o tamanho ötimo da amostra. O custo esperado, quando se coloca $\boldsymbol{I}^{-1}-1$ unidades, $\widehat{e}$ dado por:

$$
\begin{aligned}
& \text { E(custo) })_{n-1}=c_{1}^{*} \theta \sum_{i=1}^{r} \frac{1}{n-i}+c_{2}^{*}(n-1) . \quad(3.5 .3 . c) \\
& \text { A diferença das expressões }(3.5 .3 . c) \text { e (3.5.3.b) será: } \\
& \left.E \text { (custo })_{n-1}-E \text { (custo }\right)_{n}=c_{1}^{*} \theta_{0}\left(\sum_{i=1}^{x} \frac{1}{n-i}-\sum_{i=1}^{r} \frac{i}{n-i+1}\right)-c_{2}^{*} \text {, }
\end{aligned}
$$

que é igual a:

$$
c_{1}^{*} \theta_{0} \frac{r}{n(n-r)}-c_{2}^{*} .
$$


Fixando $\underline{r}, c_{1}^{*}$ e $c_{2}^{*}$ e variando $\underline{n}$ pode-se obter o tamanho ótimo da amostra. o valor de $\underline{n}$ que satisfaz esta condição ë aquele que torna a expressão (3.5.3.d) menor ou igual a zero.

o valor de $\underline{n}$ que torna a expressão (3.5.3. đ) nula $\vec{e}$ dado por:

$$
\mathrm{n}=0,5 r\left(1+\sqrt{1+4 \frac{\mathrm{c}_{1}^{x}}{\mathrm{c}_{2}^{x}} \frac{0}{\mathrm{r}}}\right),
$$

onde $\underline{\mathrm{e}} \overrightarrow{\mathrm{e}} \mathrm{o}$ menor inteiro contido.

$$
\text { Pode-se estimar } \underline{\theta} \text {, supondo } \underline{x} \text { e especificando uma variân- }
$$
cia $V$. Como $V(\theta)=\theta^{2} / r$, então $\theta=\sqrt{V \cdot r}$; substituindo esta expressão em $(3.5 .3 . e)$, tem-se:

$$
n=0,5 r\left(1+\sqrt{1+\frac{4 c_{1}^{x}}{c_{2}^{x}} \sqrt{\frac{V}{r}}}\right) .
$$

Quando a amostragem $\overrightarrow{\mathrm{e}}$ com reposição, a expressão(3.5.3.a)

ficarä:

$$
E(\text { custo })=c_{1}^{*} \frac{r_{0}^{\theta}}{n}+c_{2}^{*}(n+r-1) .
$$

Analogamente ao caso anterior, pode-se calcular $\mathrm{n}$ fixando $c_{1}^{*}, c_{2}^{*}, \underline{r}$ e variar $\underline{n}$; o tamanho da amostra ötimo é aquele que minimiza o custo esperado.

$$
\text { Quando colocamos ( } n-1) \text { unidades, o custo mẻdio esperado }
$$
é fornecido por:

$$
E \text { (custo) }{ }_{n-1}=\frac{c_{1}^{*} r \theta_{0}}{n-1}+c_{2}^{*}(n+r-2) .
$$


A diferença das expressões $(3.5 .3 . h)$ e (3.5.3.g) serä:

$E$ (custo $)_{n-1}-E$ (custo $)_{n}=c_{1}^{*} r \theta_{0}\left(\frac{1}{n-1}-\frac{1}{n}\right)-c_{2}^{*} \cdot(3.5 \cdot 3 \cdot \dot{1})$

o valor de $\underline{\mathrm{n}}$ que torna a expressão $(3.5 .3 . \dot{i})$ nula $\bar{e}:$

$$
\mathrm{n}=0,5\left(1+\sqrt{1+\frac{4 c_{1}^{*}}{c_{2}^{*}} \times \theta_{0}}\right)
$$

onde $\underline{\mathrm{n}} \overline{\mathrm{e}}$ o menor inteiro contido.

Para compararmos os custos esperados, no caso da amostragem ser com ou sem reposição, vamos definir:

$$
\mathrm{n}_{\mathrm{SR}}=\mathrm{n}_{\mathrm{CR}}+\mathrm{r}-1
$$

o nümero $\mathrm{n}_{\mathrm{CR}}$ não é necessariamente igual a $\mathrm{n}_{\mathrm{SR}}$.

$$
\text { Substituindo-se a expressão }(3.5 .3 . k) \text { na expressão }
$$

$(3.5 .3 . b)$, tem-se:

$$
\begin{aligned}
& E \text { (custo) }_{S R}=c_{1}^{*} \theta_{0} \sum_{i=1}^{r} \frac{1}{n_{S R}-i+1}+c_{2}^{*} n_{S R}, \\
& =c_{1}^{*} \theta_{0} \sum_{i=1}^{r} \frac{1}{n_{C R}+r-i}+c_{2}^{*}\left(n_{C R}+r-1\right) . \\
& \text { Das expressöes }(3.5 .3 . \ell) \text { e }(3.5 .3 . g) \text { tem-se: } \\
& \quad \frac{E \text { (custo) SR }}{c_{2}} \leqslant \frac{E(\text { custo }) C R}{c_{2}},
\end{aligned}
$$

pois:

$$
\frac{E \text { (custo) } S R}{c_{2}}=\frac{c_{1}^{*} \theta}{c_{2}^{*}}\left(\frac{1}{n_{C R}+r-1}+\frac{1}{n_{C R}+r-2}+\ldots+\frac{1}{n_{C R}}\right)+\left(n_{C R}+r-1\right)
$$




$$
\frac{E \text { (custo) }}{C R}=\frac{c_{1}^{*} \theta_{0} r}{c_{2}^{*}}-\frac{1}{{ }^{n} C R}+\left(n_{C R}+r-1\right) .
$$

Comparando-se a expressão (3.5.3.n) com a expressão (3.5.3.o), verifica-se (3.5.3.m). Portanto, o custo mẻdio esperado sem reposição è menor ou igual ao custo esperado com reposição. Se $r=1$, os custos esperados são iguais. Estes resultados foram obtidos por RILEY (1962) e BLIGHT (1972).

Quando a censura è do tipo I, hâ duas maneiras de calcu$\operatorname{lar} \underline{n}$

Assim, para um teste sem reposição, EPSTEIN (1954) apresenta uma förmula aproximada para o tamanho da amostra obtida de $p=\frac{r_{0}}{n}$ onde $p=1-\exp \left(-\frac{t^{*}}{C}\right)$. Tem-se então:

$$
\mathrm{n}=\frac{\mathrm{r}_{0}}{1-\exp \left(\frac{-t^{*}}{C}\right)}
$$

onde

$$
c=\frac{\theta_{0} \cdot x_{2 r_{0}, \alpha}^{2}}{2 r_{0}}
$$

Esta aproximação deve ser utilizada se $\theta_{\sigma} / t^{*}$ è grande, por exemplo, $\theta_{0} / t *>3$

Quando o teste $\vec{e}$ com reposição, o tamanho da amostra ê,

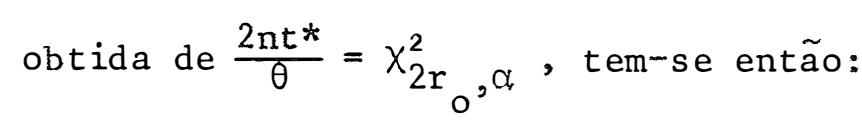

$$
\mathbf{n}=\frac{\theta_{0} x_{2 r_{0}}^{2}, \alpha}{2 t^{*}}
$$




\subsection{Anālise de Dados:}

Na maioria de nossas explanações, adnite-se que a variävel em estudo tenha distribuição exponencial. Hä vầrios testes que permitem verificar se a variável possui distribuição exponencial, o que se farä, a seguir, atravês de alguns testes.

\subsubsection{Teste Qui-quadrado:}

o teste qui-quadrado não se restringe apenas à distribui... ção exponencial. Para aplicä-lo deve-se estimar ini̊cialnente, o parâmetro $\underline{\theta}$ pelo método da mäxima verossimilhança. Dividem-se os dados en $\underline{k}$ classes e estima-se o número de unidades que caem em cada classe, sob a hipótese de que o modelo é exponencial. o nümero de classes é escolhido como sendo o maior possỉvel, sujeito à restrição de que o nümero esperado de unidades, em cada classe, seja ao menos cinco. Os limites das classes säo escolhidos de modo que o número esperado de unidades em cada classe seja igual a:

$$
\begin{aligned}
& P\left(T \leqslant t_{1}\right)=\frac{1}{k}, \\
& P\left(T \leqslant t_{2}\right)=\frac{2}{k}, \\
& \ldots \ldots \ldots \ldots \\
& P\left(T \leqslant t_{k-1}\right)=\frac{k-1}{k}, \\
& P\left(T \leqslant t_{k}\right)=\frac{1}{k},
\end{aligned}
$$

Como $P\left(T \leqslant t_{i}\right)=1-\exp \left(\frac{-t}{\theta}\right), i=1,2, \ldots, k$, pode-se assim obter os 1imites superiores das classes. 
0 nümero esperado $\vec{e} \quad e_{i}=\frac{n}{k}$ para todo $\underline{i}$.

Deve-se, então, calcular a estatística:

$$
x^{2}=\sum_{i=1}^{k} \frac{\left(o_{i}-e_{i}\right)^{2}}{e_{i}}
$$

Esta estatïstica deve ser comparada com $x_{k-2, \alpha}^{2}, \quad$ onde $x_{k-2, \alpha}^{2}$ è o valor crïtico tabelado da distribuição de qui-quadrado. Se $x^{2}>x_{k-2, \alpha}^{2}$, isto indica que o modelo exponencial é incorreto.

3.6.2 Teste para verificar se a Taxa de Falhas permanece constante:

Este teste permite detectar o crescimento ou a diminuiçăo da taxa de falhas. Estä ele baseado na estatística de Barthett's a qual estabelece que se $s_{i}^{2} ; i=1,2, \ldots k$ são estimativas independentes da mesma variância, cada uma com $n_{i}$ graus de liberdade. Assim,

$$
x^{2}=\frac{1}{1+\frac{1}{3(k-1)}\left[\sum_{i=1}^{k}\left(\frac{1}{n_{i}}\right)-\frac{1}{n}\right]} \operatorname{n\ell n}\left(\frac{\sum_{i=1}^{k} n_{i} s_{i}^{2}}{n}\right)-\sum_{i=1}^{k} n_{i} \ell n s_{i}^{2},
$$

onde $n=\sum_{i=1}^{k} n_{i}$

A expressão (3.6.2.a) possui distribuição de qui-quadrado com $k-1$ graus de liberdade.

Pois se $\frac{n_{i} s_{i}^{2}}{\sigma^{2}}$ possui distribuição de qui-quadrado com $n_{i}$ graus de liberdade, então $\sum_{i=1}^{\sigma^{2}} \frac{n_{i} s_{i}^{2}}{\sigma^{2}}$ possui distribuição de qui-quadrado 
com $\underline{\mathrm{n}}$ graus de liberdade.

Considerando-se que os tempos totais entre falhas $T\left(t_{1, n}\right)$, $T\left(t_{2, n}-t_{1, n}\right), \ldots, T\left(t_{r, n}-t_{r-1, n}\right)$ são inảependentes e que:

$$
\begin{aligned}
& \frac{2 T\left(t_{1, n}\right)}{\theta} \sim x_{2}^{2}, \\
& \frac{2 T\left(t_{i, n}-t_{i-1, n}\right)}{\theta} \sim x_{2}^{2}, \quad i=1,2, \ldots, r, \\
& \frac{2 T\left(t_{r, n}\right)}{\theta} \sim x_{2 r}^{2} .
\end{aligned}
$$

onde $T_{i, n}=T\left(t_{i, n}\right)=t_{1, n}+t_{2, n}+\ldots+(n-i+1) t_{i, n} \quad e$ $T\left(t_{i, n}-t_{i-1, n}\right)=(n-i+1)\left(t_{i, n}-t_{i-1, n}\right)$.

Pode-se, assim, reescrever (3.6.2.a), usando-se o fato

que $n_{i} S_{i}^{j}$ e $T\left(t_{i, n}-t_{i-1, n}\right)$ ambas possuem distribuição de qui-quadrado. $B_{r}=\frac{2 r\left\{\ln \frac{T\left(t_{r, n}\right)}{r}-\frac{1}{r}\left[\operatorname{lnT}\left(t_{1, n}\right)+\operatorname{lnT}\left(t_{2, n}-t_{1, n}\right)+\ldots+\operatorname{lnT}\left(t_{r, n}-t_{r-1, n}\right)\right]\right.}{1+\frac{(r+1)}{6 r}}$.

$(3.6 .2 . c)$

A estatística $B_{r}$ possui distribuição aproximadamente qui-quadrado com $r-1$ graus de liberdade. Rejeita-se a validade da distribuição exponencial, se $\mathrm{B}_{\mathrm{r}}>\mathrm{X}_{\mathrm{r}-1, \alpha^{2}}^{2}$ O valor crïtico $X_{r-1, \alpha}^{2}$ è obtido da tabela da distribuição qui-quadrado.

\subsection{3 - Técnicas Gräficas}

Uma considerāvel importância deve ser atribuïda às tëcnicas gräficas para a compreensão de processos probabilísticos. 
Um gräfico bem traçado pode fornecer informações bastan te satisfatörias. De (3.1.b) tem-se que

$$
F(t)=F(t ; \theta, \delta)=1-\exp \left[\frac{-(t-\delta)}{\theta}\right],
$$

então,

$$
1-F(t)=\exp \left[\frac{-(t-\delta)}{\theta}\right] .
$$

Tomando-se o logaritmo na base natural, tem-se que:

$$
y=\ln \frac{1}{1-F(t)}=\frac{t-\delta}{\theta} .
$$

Para (3.1.d), tem-se que:

$$
F(t)=F(t ; \theta)=1-\exp \left(\frac{-t}{\theta}\right) ;
$$

então,

$$
1-F(t)=\exp \left(\frac{-t}{\theta}\right)
$$

Tomando-se o logaritmo na base natural, ter-se-âa:

$$
y=\ln \left[\frac{1}{1-F(t)}\right]=\frac{t}{\theta}
$$

Se a função de densidade é exponencial, obtẻm-se uma reta com declive $\mathrm{m}=\frac{1}{\theta}=\lambda$. Para (3.6.3.a) a reta corta o eixo da abscissa (t); para (3.6.3.b) a reta passa pela origem.

Existem vārias formas de estimar-se a função distribuição e uma delas ê:

$$
\hat{F}\left(t_{i}\right)=\frac{i-\frac{1}{2}}{n},
$$


onde $\underline{i}$ e a $\underline{i}$-ésima estatỉstica de ordem. Este mëtodo atribui peso igual para cada observação. Observa-se que a estimativa da função distribuição não assume os valores zero e um. o segundo método para estimar a função distribuição ê devido a GUMBEL (1958).

A estimativa da função distribuição por ele usada è:

$$
\hat{F}\left(t_{i}\right)=\frac{i}{n+1},
$$

onde $\underline{i}$ è a î-ésima estatistica de ordem. 0 mêtodo de Gumbel atribui maior peso aos pontos extremos do grä́fico, ou seja, os pontos limites assumem um peso maior. Isto significa que eles estão mais afastados de zero e um, do que no primeiro método citado (3.6.3.c).

HAHN e SHAPIRO (1967) observam que uma forma aproximada do valor esperado de um conjunto de observaçöes pode ser expresso por $(i-c) /(n-2 c+1)$. O melhor valor de $c$ depende da função de densidade e do tamanho da amostra. Eles observam, ainda, que o valor de $c=0 \hat{e}$ me1hor, quando a função de densidade ê uniforme; $c=0$ leva ao método (3.6.3.d). Finalmente, notam que $c=1 / 2$ tem sido geralmente aceitâvel para uma grande variedade de distribuiçôes e tamanhos de amostras; $c=1 / 2$ leva ao mêtodo (3.6.3.c). 


\section{EXEMPLOS NUMERICOS}

4.1 - Distribuição Exponencial Monoparamētrica

\subsection{1 - Censura Tipo II - Amostragem sem reposição}

Um teste de vida è executado com cinqüenta unidades de um determinado produto. A amostragem é censurada após a décima falha e as unidades que falham não são repostas. Os tempos de falha são medidos em semanas e os instantes de falha são: 0,$2 ; 0,4 ; 0,6 ; 0,9 ; 1,2 ; 1,3 ; 1,9$; 2,$0 ; 2,1$ e 2,6 .

Pode-se determinar a estimativa pontual para a vida mëdia. Da expressão (3.2.1.e) ter-se-ä:

$$
\hat{\theta}=\frac{13,2+40 \cdot 2,6}{10}=11,720 .
$$

Da expressão (3.2.1.t) obtēm-se o intervalo de confiança para a vida mêdia. Supondo $\alpha=0,05$ tem-se: 


$$
P\left(\frac{2 \cdot 10 \cdot 11,72}{31,410} \leqslant \theta \leqslant \frac{2 \cdot 10 \cdot 11,72}{10,851}\right)=0,90 \text {, }
$$

$\mathrm{d} a .1$

$$
P(7,463 \leqslant \theta \leqslant 21,602)=0,90 .
$$

Portanto a probabilidade de que a vida média se encontre nesse intervalo è igual a 0,90 .

$$
\text { Pode-se estar interessado em realizar o seguinte teste }
$$

$\mathrm{H}_{0}: \theta \geqslant 15$ versus $\mathrm{H}_{1}: \theta<15$. Da tabela 5 do Apêndice, obtën-se a esta tistica teste. Substituindo os valores numëricos tem-se:

$$
x_{\mathrm{emp}}^{2}=\frac{2 \cdot 10 \cdot 11,72}{15}=15,63 .
$$

Da tabela da distribuição de qui-quadrado tem-se o valor crïtico para $\alpha=0,05$ que é igual à 10,851. Como $\chi_{\text {emp }}^{2}>10,851$ aceita-se a hipótese $\mathrm{H}_{\mathrm{O}}$, ao nivel de significância de 0,05 .

Para desenhar a curva característica de operação, deve-se utilizar a expressão $(3 \cdot 5 \cdot 1 \cdot j)$. Calcula-se $P_{a}(\theta)$ para alguns valorłz de Q, supondo-se $\theta_{0}=15$ e $\alpha=0,05$. Tem-se então:

$$
\begin{aligned}
& P_{a}(15)=P\left(X_{20}^{2} \geqslant 10,851\right)=0,95 . \\
& P_{a}(10)=P\left(X_{20}^{2} \geqslant 16,277\right) \cong 0,70 . \\
& P_{a}(7,5)=P\left(X_{20}^{2} \geqslant 21,702\right) \cong 0,36 . \\
& P_{a}(5,0)=P\left(X_{20}^{2} \geqslant 32,553\right) \cong 0,03 .
\end{aligned}
$$




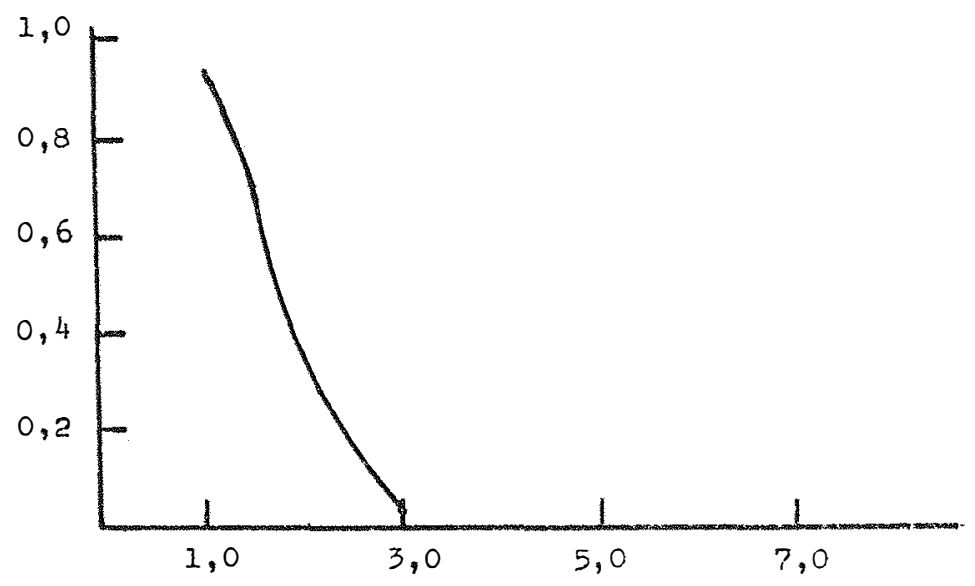

Atravês dessa curva, pode-se determinar a probabilidade de aceitar a hipötese $H_{0}$ para alguns valores de $\theta_{0}$ e $\theta_{1}$.

o tempo médio esperado para a execuçăo do experimento pode ser obtido da tabela 6 do Apêndice. Desta, para $n=50$ e r $=10$ temse que $1 / \theta_{0} E\left(T_{10,50}\right)=0,22066$, como $\theta_{0}=15$, então o tempo mêdio esperado para a execução do experimento $\vec{e}$ de 3,31 semanas.

Da tabela 7 do Apêndice, determina-se a economia do tempo do teste. Para $n=50$ e $r=10$, obtêm-se $E_{f}$ igual a 0,07534; o experimento terā apenas $7 \%$ da duração do tempo em relaçāo ao experimento no qual são colocadas dez unidades, e espera-se que todas falhem.

$$
\text { Utilizando-se apenas as cinco primeiras falhas pode-se }
$$
calcular novamente a estimativa pontual da vida mẻdia. T'em-se:

$$
\hat{\theta}=\frac{3,3+45 \cdot 1,2}{5}=11,450 .
$$


O erro percentual que estā sendo cometido, utilizando-se as primeiras cin co falhas em vez das dez primeiras falhas $\hat{e}$ de

$$
\frac{100(11,720-11,460)}{11,720}=2,22 \% \quad .
$$

Se o custo de observação por unidade de tempo é de 2 u.m. (unidade monetārias) e o custo para colocar uma unidade no experimento è de 1 u.m., pode-se então dimensionar o tananho ötimo da amostra que mini miza os custos. Mantendo-se $\mathrm{r}=10 \mathrm{e} \theta_{\mathrm{o}}=15$ da expressão (3.5.3.e) tem-se:

$$
\mathrm{n}=\left[10 \cdot 0,5\left(1+\sqrt{1+4 \cdot 2 \cdot \frac{15}{10}}\right)\right]=23
$$

o custo total do experimento è mínimo se observarmos dez falhas em uma amostra de vinte três unidades.

\subsection{2 - Censura tipo II. Amostragem com reposição}

Um teste de vida è executado com vinte cinco unidades de um determinado produto. A amostragem é censurada apôs a décima falha. Os tempos de falha são medidos em horas e a ültima falha ocorre no instante $470 \mathrm{~h}$, havendo reposição das unidades que falham.

A estimativa pontual da vida mẻdia é dada pela expressâo $(3.2 .2 . b)$. Assim:

$$
\hat{\theta}=\frac{25 \cdot 470}{10}=1175
$$


Da expressão (3.2.2.e), adotando-se $\alpha=0,10$ tem-se:

$$
\frac{p(2 \cdot 25 \cdot 470}{31,410} \leqslant \theta \leqslant \frac{2 \cdot 25 \cdot 470}{10,851}=0,90
$$

da $\vec{i}$

$$
P(748,169 \leqslant \theta \leqslant 2165,699)=0,90 .
$$

Portanto, a probabilidade de que a vida mêdia esteja contida neste intervalo é igual a 0,90 .

Da expressão (3.5.1.z), obtêm-se a economía de tempo da duração do experimento. O valor de $E_{E} \vec{e} 0,40$, portanto o experimento terá apenas $40 \%$ da duração, em relação a um experimento onde são colocadas dez unidades e espera-se que dez falhas ocorram.

Para o teste $\mathrm{H}_{0}: \theta \leqslant 1200$ versus $\mathrm{H}_{1}: \theta>1200$, obtêmse da tabela 5 do Apêndice a estatỉstica teste. Substituindo-se os valores numéricos tem-se:

$$
x_{\mathrm{emp}}^{2}=\frac{2 \cdot 10 \cdot 1175}{1200}=19,58
$$

Da tabela da distribuição de qui-quadrado, tem-se o valor crîtico para $\alpha=0,05$; que ê 10,851. Como $\chi_{\text {emp }}^{2}>10,851$ deve-se rejeitar a hipótese $H_{0}$ ao nîvel de significância de 0,05.

Se o custo de observação por unidade de tempo ê de $2 \mathrm{u} \cdot \mathrm{m}$. e o custo para colocar uma unidade no experimento $\vec{e}$ de $1 \mathrm{u} \cdot \mathrm{m}$.; pode-se dimensionar o tamanho ótimo da amostrà que minimiza estes custos. Mantendo-se $r=10$ e $\theta_{0}=1200$; da expressão $(3.5 .3 . j)$, tem-se:

$$
\mathrm{n}=\left[0,5\left(1+\sqrt{1+\frac{4 \cdot 2 \cdot 10 \cdot 1175}{1}}\right)\right]=153 .
$$


o custo total do experimento é mintimo, se observarmos dez falhas em uma amostra de cento e cinqüenta e três unidades.

\subsection{3 - Censura tipo I. Amostragem com reposição}

Um teste de vida ê executado com quarenta e nove unidades. A amostragem ë censurada para $t^{*}=40 \mathrm{~h}$. As unidades que falham não são repostas. Durante o intervalo de tempo de $40 \mathrm{~h}$ ocorreram 15 falhas.

A estimativa pontual da vida mëdia é obtida da expressão $(3 \cdot 2.4 . d)$, ou seja:

$$
\hat{\theta}=\frac{49 \cdot 40}{15}=130,6 \mathrm{~h}
$$

o intervalo de confiança é obtido da expressão (3.2.4.p). Portanto, para $\alpha=0,10$ tem-se:

$$
P\left(\frac{2 \cdot 1960}{46,194} \leqslant \theta \leqslant \frac{2 \cdot 1960}{18,493}\right)=0,90,
$$

$\mathrm{da}$

$$
P(84,860 \leqslant \theta \leqslant 211,972)=0,90 .
$$

A probabilidade de que a vida mëdia esteja contida resse intervalo $\vec{e}$ igual a 0,90 .

Para se estimar a confiabilidade deve-se utilizar a expressão (3.3.b). Se $t=100 \mathrm{~h}$ ter-se-ä: 


$$
\hat{C}(100)=\left(1-\frac{100}{1960}\right)^{14}=0,480 .
$$

Portanto, a probabilidacie de que uma unidade permaneça funcionando apös $100 \mathrm{~h}$ ê 0,480 .

O intervalo de confiança para essa confiabilidade pode ser obtido da expressāo (3.3.e), ou seja:

$$
\mathrm{P} \cdot\left[\exp \left(-\frac{100}{84,860}\right) \leqslant C(100) \leqslant \exp \left(-\frac{100}{211,972}\right)\right]=0,90,
$$

dai :

$$
P(0,308 \leqslant C(100) \leqslant 0,624)=0,90 .
$$

Portanto, a probabilidade de que a confiabilidade para $t=100 \mathrm{~h}$ esteja contida neste intervalo $\vec{e}$ igual a 0,90 .

\section{1 .4 - Censura tipo I. Amostragem sem reposição}

Em um teste de vida foram colocadas quarenta e nove unida des. O teste è censurado para $t^{*}=40 \mathrm{~h}$, e as unidades que falham não säo repostas. Os instantes das falhas são: 1,2;2,2;4,9;5,0;6,8;7,0; 12,$8 ; 13,7 ; 15,1 ; 15,2 ; 23,9 ; 24,3 ; 25,1 ; 35,8$ e $38,9 \mathrm{~h}$.

A estimativa da vida mêdia è dada pela (3.2.3.b). Tem-se:

$$
\hat{\theta}=\frac{231,9+(49-15) 40}{15}=106,127 .
$$

Para determinar o intervalo de confiança, usa-se a expres são $(3 \cdot 2 \cdot 3 \cdot k)$. 
Inicialmente deve-se calcular o Iimite superior " $S$, que é dado em (3.2.3.h). Para $\alpha=0,05$ e $\mathrm{n}=49$, obtëm-se $\mathrm{p}_{\mathrm{s}}=0,42$.

Analogamente determina-se o limite inferior $\mathrm{p}_{\tau}$ pela expressão (3.2.3.i); obtēm-se $p_{I}=0,20$. Substituindo-se $p_{S}$ e $p_{I}$ em $(3.2 .3 \cdot k)$, tem-se:

$$
P(73,431 \leqslant \theta \leqslant 179,257)=0,90 .
$$

Assim, a probabilidade de que a vida mêdia esteja contida no intervalo $\vec{e}$ igual a 0,90 .

Para determinar uma estimativa pontual da confjabilidade, usa-se a expressão (3.3.b). Para $t=100$ h tem-se:

$$
\hat{C}(100)=\left(1-\frac{100}{15 \cdot 106,127}\right)^{14}=0,403 .
$$

Portanto, a probabilidade de que uma unidade permaneça funcionando apōs 100 h é 0,403.

\section{2 - Distribuição Exponencial Biparamêtrica}

Um teste de vida $\widehat{e}$ executado com vinte unidades em cond $\dot{i}-$ ções semelhantes. A amostragem ê censurada após a dẹcima falha, não haven do reposição das unidades que falham. Os tempos de falhas são medidos em horas, e os instantes de falhas são: 203; 246;279; 306; 346; 394; 411; $417 ; 519 ; 535$. 
Estes dados provêm de uma distribuição exponencial com ví da mỉnima não nula.

Para determinar as estimativas da vida mêdia e vida mínima, utiliza-se a expressão (3.4.m):

$$
\tilde{\theta}=\frac{1626+10 \cdot 332}{9}=549,556 \text {, }
$$

e

$$
\gamma=203-\frac{549,556}{20}=175,522 .
$$

- limite de confiança inferior para a vida mínima é obtido da expressão (3.4.0). Adotando-se $\alpha=0,05$ tem-se:

$$
P\left(\delta \geqslant 203-\frac{549,556}{20} \cdot 7,21\right)=0,95,
$$

$\mathrm{da} \vec{I}$

$$
P(\delta \geqslant 4,885)=0,95 \text {. }
$$

Desde que $\delta>0$, isto suggere que a distribuição exponencial monoparamétrica não ê um modelo adequado.

Da expressão (3.4.p) obtêm-se o intervalo de confiança para a vida mẻdia. Adotando-se $\alpha=0,10$ tem-se:

$\mathrm{da} \vec{i}$

$$
\mathrm{P}\left(\frac{2.9 .549,556}{28,869} \leqslant \theta \leqslant \frac{2.9 .549,556}{9,390}\right)=0,90
$$

$$
P(342,652 \leqslant \theta \leqslant 1053,462)=0,90 .
$$

A probabilidade de que a vida mêdia esteja contida nesse intervalo $\vec{e}$ igual a 0,90 . 
4.3 - Anâlise de dados

\subsection{1 - Teste Qui-Quadrado}

Um teste de vida ê executado com cem unidades de um deter minado produto. A amostragem ê censurada apôs a décima quinta falha e as unidades que falham não são repostas. Os instantes de falha em horas são: $220 ; 455 ; 620 ; 974 ; 1240 ; 1290 ; 1412 ; 1950 ; 2165 ; 2691 ; 3025 ; 3808 ; 4520$; $6003 ; 6935 \mathrm{~h}$.

Para se verificar a validade do modelo exponencial a esses dados, utilizar-se-á o teste qui-quadrado, 3.6.1.

Deve-se, inicialmente, estimar o parâmetro $\underline{\theta}$. Da expressão (3.2.1.e) tem-se:

$$
\hat{\theta}=\frac{37308+35(6935)}{15}=18668,87 \mathrm{~h} .
$$

Decide-se agrupar esses dados em quatro classes;cada clas se com cinco dados e a ültima classe com os restantes.

Para determinar os limites dessas classes usa-se a expres são $\hat{t}_{p}=-\hat{\theta} \ln (\cdot 1-p) \quad$ tem-se:

$$
\begin{aligned}
& t_{0,05}=-18668,87 \ln (0,95)=957,59 . \\
& t_{0,10}=-18668,87 \ln (0,90)=1966,96 . \\
& t_{0,15}=-18668,87 \ln (0,85)=3034,04 .
\end{aligned}
$$

Pode-se construir a tabela 


\begin{tabular}{cccc}
\hline classes & $e_{i}$ & $o_{i}$ & $\left(e_{i}-o_{i}\right)^{2} / e_{i}$ \\
\hline $0 \leftarrow 957,59$ & 5 & 3 & 0,8 \\
$957,89 \leftarrow 1966,96$ & 5 & 5 & 0 \\
$1966,96 \leftarrow 3034,04$ & 5 & 3 & 0,8 \\
$3034,04 \leftarrow$ & 85 & 89 & $\frac{0,19}{1,79}$ \\
\hline
\end{tabular}

Assim $x_{\text {emp }}^{2}=1,79$. Da tabela da distribuição de qui-quadra do tem-se que o valor critico para $\alpha=0,05$; que $\vec{e}$ igual a 5,991. Como $\chi_{\text {emp }}^{2}<5,991$ não se deve rejeitar a validade do modelo exponencial.

\subsection{2 - Teste para verificar se a taxa de falhas permanece constante}

Para os mesmos dados de 4.1.1. pode-se verificar se a taxa de falhas permanece constante, utilizando-se para isto a expressão (3.6.2.c). Inicialmente calcula-se o tempo total do teste e os respectivos logaritmos naturais.

\begin{tabular}{llc}
\hline$t_{i}$ & $T\left(t_{i}-t_{i-1}\right)$ & $\operatorname{lnt}\left(t_{i}-t_{i-1}\right)$ \\
\hline 0,2 & $50(0,2)=10$ & 2,3026 \\
0,4 & $49(0,2)=9,8$ & 2,2824 \\
0,6 & $48(0,2)=9,6$ & 2,2618 \\
0,9 & $47(0,3)=14,1$ & 2,6462 \\
1,2 & $46(0,3)=13,8$ & 2,6247 \\
1,3 & $45(0,1)=4,5$ & 1,5041 \\
1,9 & $44(0,6)=26,4$ & 3,2734 \\
2,0 & $43(0,1)=4,3$ & 1,4584 \\
2,1 & $42(0,1)=4,2$ & 1,4351 \\
2,6 & $41(0,5)=20,5$ & 3,0204 \\
\hline
\end{tabular}


Substituindo em 3.6.2.c tem-se:

$$
\mathrm{B}_{20}=\frac{2 \cdot 10\left[\ln \left(\frac{116,8}{10}\right)-\frac{22,793872}{10}\right]}{1+\frac{(10+1)}{6 \cdot 10}}=3,02
$$

Da tabela da distribuição de qui-quadrado obtém-se $\chi_{9 ; 0,05}^{2}=$ $=16,919$. Como $\mathrm{B}_{20}<16,919$, näo se deve rejeitar a validade do modelo ex ponencial.

\subsection{3 - Técnica Gräfica}

Para verificar a validade do modelo exponencial, pode-se utilizar a tëcnica gräfica 3.6.3, aos dados do exemplo 4.1.1.

Para estimar a função distribuição utiliza-se a expressão (3.6.3.c) e apôs estima-se y atravēs da expressão (3.6.3.b). Daỉ temse

\begin{tabular}{ccc}
\hline$t_{i}$ & $\frac{1}{1-\hat{\hat{F}}\left(t_{i}\right)}$ & $y=\ln \frac{1}{1-\hat{F}\left(t_{i}\right)}$ \\
\hline 0,2 & 1,0200 & 0,02 \\
0,4 & 1,0408 & 0,04 \\
0,6 & 1,0625 & 0,06 \\
0,9 & 1,0851 & 0,08 \\
1,2 & 1,1087 & 0,11 \\
1,3 & 1,1333 & 0,13 \\
1,9 & 1,1591 & 0,15 \\
2,0 & 1,1860 & 0,17 \\
2,1 & 1,2143 & 0,19 \\
2,6 & 1,2439 & 0,22 \\
\hline
\end{tabular}

Agora deve-se representar estes resultados no gräfico. 
88.

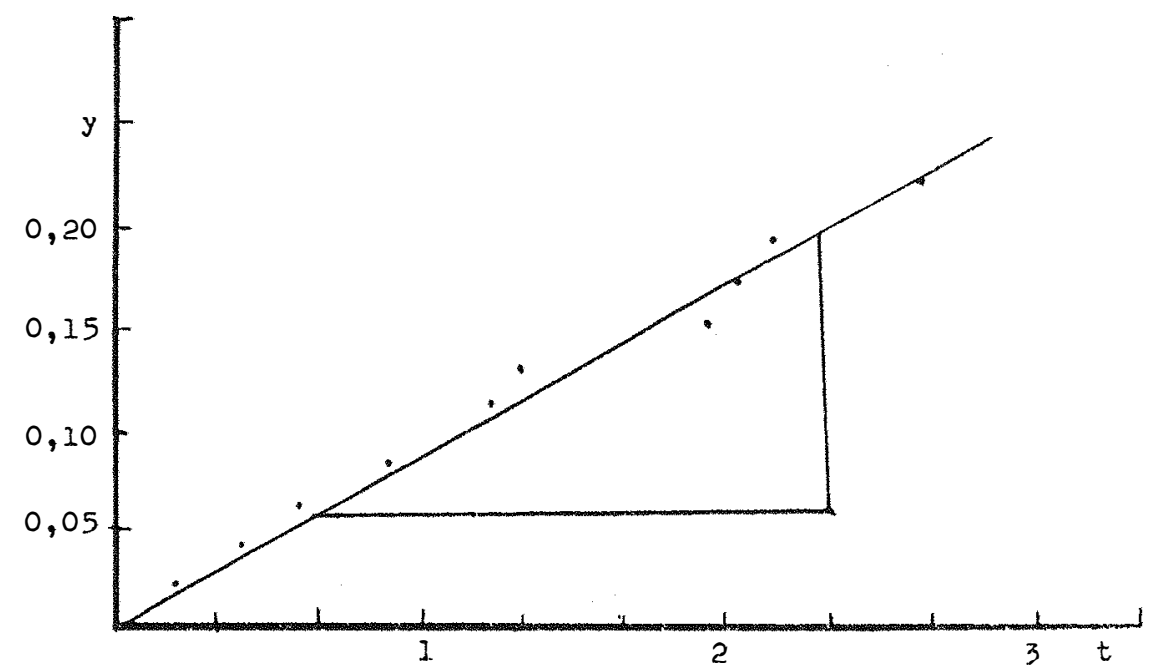

A estimativa gräfica para a vida média é dada pela expres são $(3.6 .3 . b)$, tem-se

$$
\mathrm{m}=\frac{1}{\hat{\theta}}=\frac{2,7 \times 3}{5 \times 20}=0,081 \text {, }
$$

$\operatorname{da} \hat{i} \hat{\theta}=12,35$. 


\section{CONCLUSÕES}

Com base no estudo realizado, após resultados e discussão, e apoiando-se na condição de que a teoria desenvolvida só pode ser aplicada a dados de teste de vida, desde que seja välido o modelo exponencial, pode-se concluir:

a) A interrelação entre a distribuição exponencial e a distribujição de Poisson é muito útil no desenvolvimento da teoria de testes de vida.

b) Quando a distribuição dos tempos de falhas é conhecida, utilizase esta informação para estimar os parāmetros da distribuição. Porēm, quando aquela for desconhecida, então serā ūtil ter métodos que não dependam desta restrição.

c) A têcnica gräfica, usando-se o papel monolog ou o papel próprio, pode fornecer informaçöes bastante satisfatörias para verificicar a validade do modelo exponencial e estimar os parâmetros, porém o mêtodo de māxi- 
ma verossimilhança, que utilizou-se para estimar os parâmetros, é bastante simples e possui propriedades assintóticas desejadas.

d) E de particular importância verificar o erro que ocorre, quando a hipötese relativa à distribuição de falhas è feita atravês de um conjun to de dados insuficientes.

e) As duas tēcnicas de censura tipo I e tipo II ñäo devem ser comparadas, pois envolvem conceitos distintos.

f) A censura tipo II è utilizada em menos situações práticas, pois o tempo de duração do experimento è aleatōrio. Entretanto, è a que apresenta maior facilidade na inferência dos parāmetros, intervalos de confiança, e assim por diante.

g) Para a censura tipo II, verifica-se que o tempo necessärio para observar $\underline{r}$ falhas em uma amostra com $\underline{n}$ unidades é menor que o tempo neces sário para observar $\underline{r}$ falhas em uma amostra com $\underline{r}$ unidades. A desvantagem desta, é que n-r unidades devem ser colocadas em teste. Portanto, esta censura deverả ser empregada desde que seja comparado o custo relativo do tempo de experimento em relação ao custo da amostragen e teste de unidades extras.

h) Para a censura tipo II, o custo mẻdio esperado, quando o teste $\bar{e}$ sem reposição, $\vec{e}$ menor ou igual ao custo mëdio esperado de um teste com reposição.

i) A curva caracterîstica de operação não depende do tamanho da amos tra, mas sim do nümero de falhas observadas. Isto significa que a mesma 
precisão $\vec{e}$ obtida, quer se somente $\underline{r}$ unidades são testadas até falharem ou se $\underline{n}$ unidades são colocadas no teste e o teste terminarâ no instante em que ocorre a $\underline{r}$-ēsima falha. Além disso, pode-se determinar o nümero médio de falhas necessārias.

j) Quanto maior for o nümero de unidades colocadas em teste, menor serä o tempo médio esperado para a sua execução. 


\section{LITERATURA CITADA}

BARTHOLOMEW, D.J., 1957. Testing for departure from the exponential distribution. Biometrika. Londres, 44: 253-256.

BARTHOLOMEW, D.J., 1963. The sampling distribution of an estimate arising in 1ife-testing. Technometrics. Washington, 5: 361-374.

BASU, A.P., 1964. Estimates of reliability for some distributions usefuI in life testing. Technometrics. Washington, 6: 215-219.

BAZOVSKY, I., 1961, Reliability Theory and Practice. Nova Jersey, Prentice Ha11, Englewood Cliffs. 292 p. (Prentice-Hall Space Technology Series).

BLIGHT, B.J., 1972. On the most economical choice of a life testing procedure for exponentially distributed data. Technometrics. Washington, 4 : $140-142$.

BULGREN,W.G. e J.E. HEWETT, 1973. Double sample tests for hypotheses about the mean of an exponential distribution. Technometrics. Washington, 15: 187-190. 
COHEN, A.C., F.R. HELM, 1973. Estimation in the exponential distribution, Technometrics. Washington, 15: 415-418.

COX, D.R., 1953. Some simple approximate testes for Poisson variates. Biometrika. Londres, 40: 354-360.

EPSTEIN, B., 1954. Truncated 1 ife tests in the exponential case. The Annals of Mathematical Statistics. Baltimore, 25: 555-564.

EPSTEIN, B., 1960. Tests for the validity of the assumption that the underlying distribution of life is exponential. Part I. Technometrics. Washington, 2: 83-10i.

EPSTEIN, B., 1960. Tests for the validity of assumption that the underlying distribution of Iife is exponential. Parte II. Technometrics. Washington, 2 : 167-183.

EPSTEIN, B., 1960. Statistical life test acceptance procedures.

Technometrics. Washington 2: 435-446.

EPSTEIN, B., 1960. Estimation from 1ife test data. Technornetrics.

Washington, 2: 447-454.

EPSTEIN, B. e M. SOBEL, 1953. Life testing. Journal of the American

Statistical Association. Washington, 48: 486-502.

EPSTEIN, B. e M. SOBEL, 1954. Some theorems relevant to life testing from an exponential distribution. The Annals of Mathematical

Statistics, Baltimore, 25: 373-381.

EPSTEIN, B. e C.K. TSAO, 1953. Some tests based on ordered observations

from two exponencial populations. The Annals of Mathematical.

Statistics. Baltimore, 24: 458-554.

FERCHO, W.W. e L.J. RINGER, 1972. Smal1 sample power of some tests of the constant failure rate. Technometrics Washington, 14: 713-724. FINKLESTEIN, J.M. e R.E. SCHAFER, 1971. Improved Goodness of Fit Tests, Biometrika. Londres, 58: 641-645.

FISHER, R.A., 1922. On the mathematical foundations of theorical statistics. Philosophical Transactions of the Royal Society of London. London, vo1. 20A: 175-240 e 263-294. 
GNEDENKO, B. et BELJACO, JU. K.; SOLOVIEV, A.D., 1969. Mathematica1 Methods of Reliability Theory. Nova York, Academic Press.

GUMBEL, E.J., 1958. Statistics of Extremes. Columbia University Press. Nova York, 375 p.

HAHN, G.J. e S.S. SHAPIRO, 1967. Statistical Models in Engineering. Nova York, John Wiley \& Sons, Inc., 355 p.

HALPERIM, M., 1952. Maximum likelihood estimation in truncated samples. The Annals of Mathematical Statistics, Baltimore, 23: 226-238.

HARTLEY, H.O., 1950. The maximum F-ration as a short-cut test for heterogeneity of variance. Biometrika. Londres, 37: 308-312.

JACOBSON, P.H., 1947. The relative power of three statistics Eor smal1 sample distructive tests. Journal of the American Statistical Association. Washington, 42: 575-584.

KUMAR, S. E H.I. PATEL, 1971. A test for the comparison of two exponential distributions. Technometrics. Washington, 13: 183-189.

LILLIEFORS, H.W., 1969. On the Kolmogorov-Smirnov test for the exponential distribution with mean unknown. Journal of the American. Statistical Association." Washington, 64: 387-389.

MANN, N.R., 1969. Optimum estimators for linear functions of location and sacale parameters. The Annals of Mathematical Statistics. Baltimore, 40: 2149-2159.

MANN, N.R., SCHAFER, R.E., e SINGPURWALLA, M.D., 1974。 $\underline{\text { Methods for }}$ Statistical Analysis of Reliability and Life Data. Nova York, 564 p. MIRSHAWKA, V., 1979. O Modelo de Weibull e Aplicações à Confiabilidade. São Paulo, USP, 238p. (Dissertação de Mestrado)

MOOD, A.M., GRAYBILL, F.A., e D. BOES, 1974. Introduction to the Theory. of Statistics. Nova York, McGraw-Hil1. 564p.

MORAN, P.A.P., 1951. The randon division of an interval. Part II. Journal Royal Statistic Society, B. Londres, 13: 147-150. 
NEYMAN, J. e E.S. PEARSON, 1928. On the use and interpretation of certain best criteria for purposes of statistical inference. Biometrika. Londres, vo1. 20A: 175-240 e 263-294.

NEYMAN, J., 1935. On the problem of confidence intervalos. The Annals of Mathematical Statistics, Baltimore, 6: 111-116.

PAULSON, E., 1941. On certain Jikelihood ratio test associated with the exponential distribution. The Annals of Mathematical Statistics. Baltimore, 12: 301-306.

PUGH, E.L., 1963. The best estimate of reliability in the exponential case. Journal of the Operations Research Society of American. Baltimore, 11: 57-61.

RILEY, J.D., 1962. Comparative cost of two life test procedures. Technometrics. Washington, 4: 140-142.

SARHAN, A.E., 1954. Estimation of the mean an standard deviation by order statistics. The Amnals of Mathematical Statistics. Baltimore, 25: $317-328$.

SUKHATME, P.V., 1936. On the analysis of $k$ samples from exponential reference to the problem of random intervals. Statistical Research. Memoirs, Universidade de Londres, 1: 94-112.

ZACKS, S. e M. EVEN, 1966. The efficiencies in small samples of the maximum likelihood and best umbiased estimators of reliability function. Journal of the American Statistical Association. Washington, 61: 1033-1051.

ZELEN, M. e M.C. DANNEMILLER, 1961. The robustness of 1 ife testing procedures derived from the exponential distribution. Technometrics. Washington, 3: 29-49. 
96.

APENDICE 
FIGURAS - Curvas características de operação para alguns testes

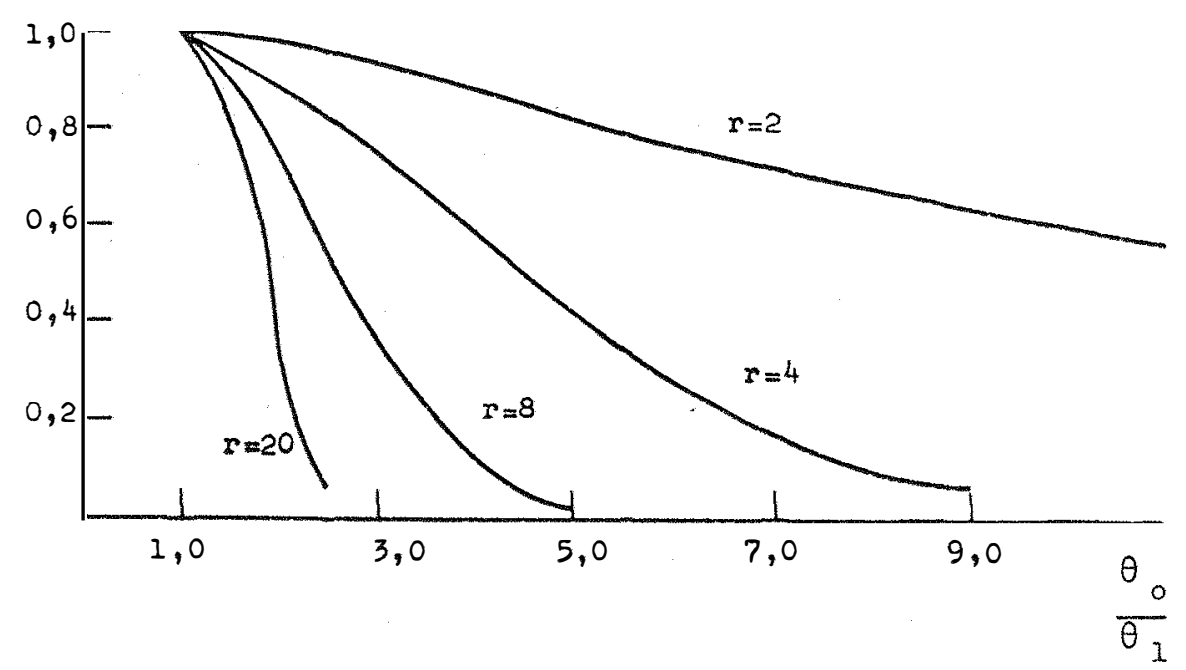

Teste para $H_{0}: \theta \geqslant \theta_{0}$ versus $H_{1}: \theta<\theta_{0} \quad \alpha-0,01$

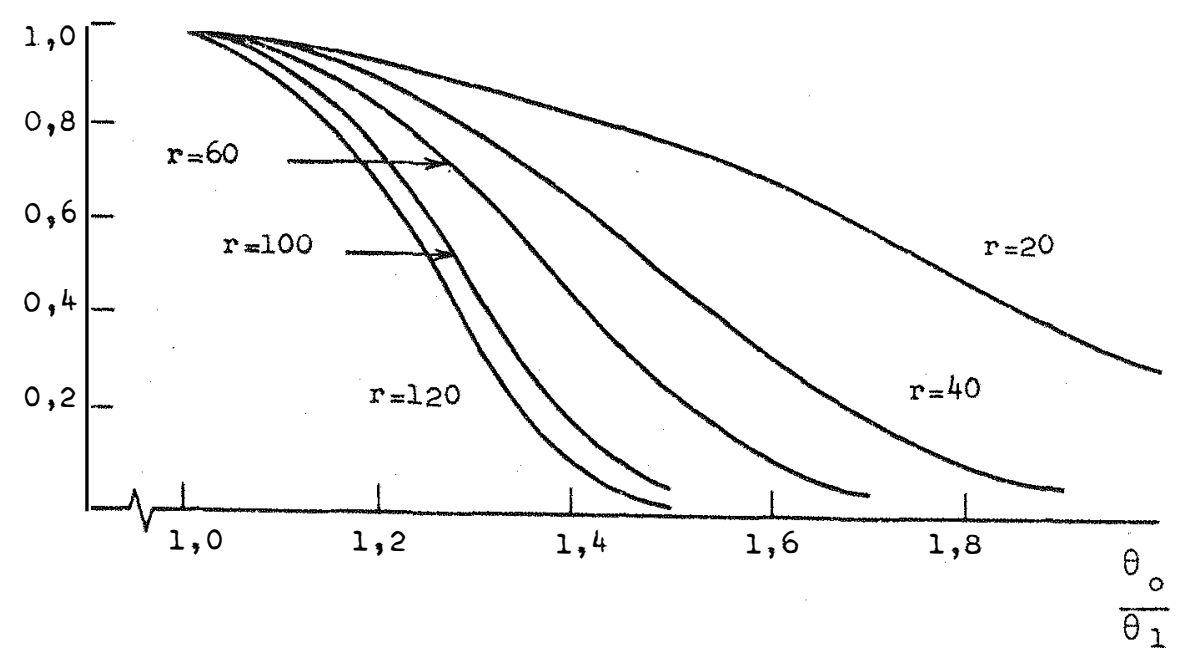

Teste para $\mathrm{H}_{0}: \theta \geqslant \theta_{0}$ versus $\mathrm{H}_{1}: \theta<\theta_{0} \quad \alpha=0,01$ 


\section{Figuras - continuação}

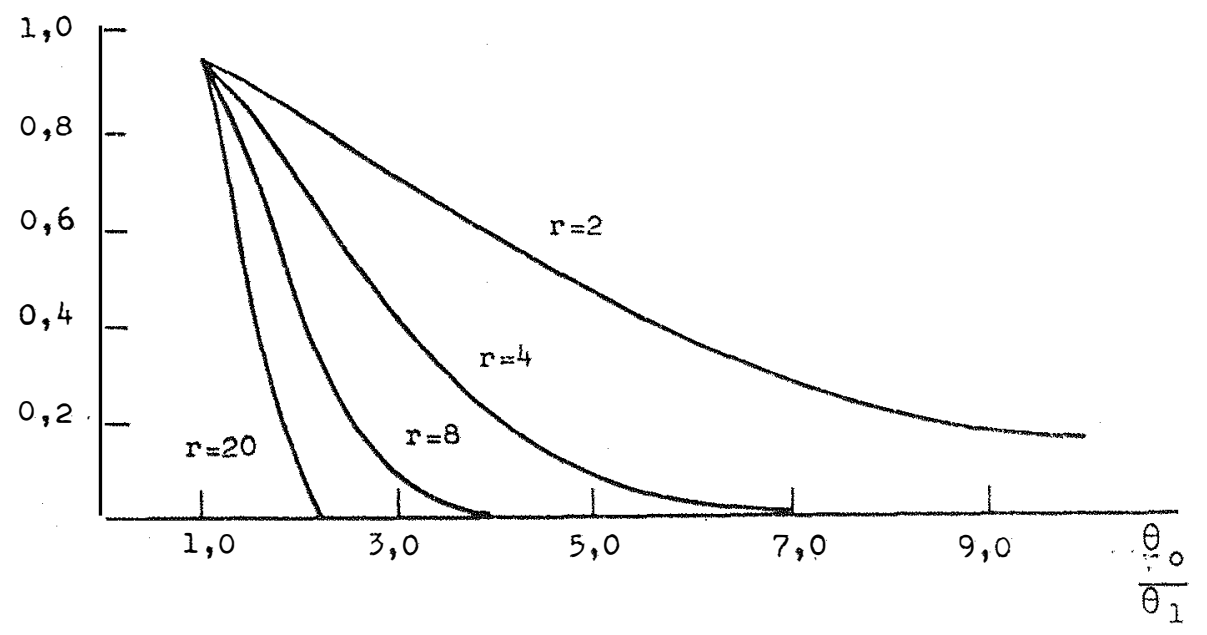

Teste para $H_{0}: \theta \geqslant \theta_{0}$ versus $H_{1}: \theta<\theta_{0} ; \alpha=0,05$

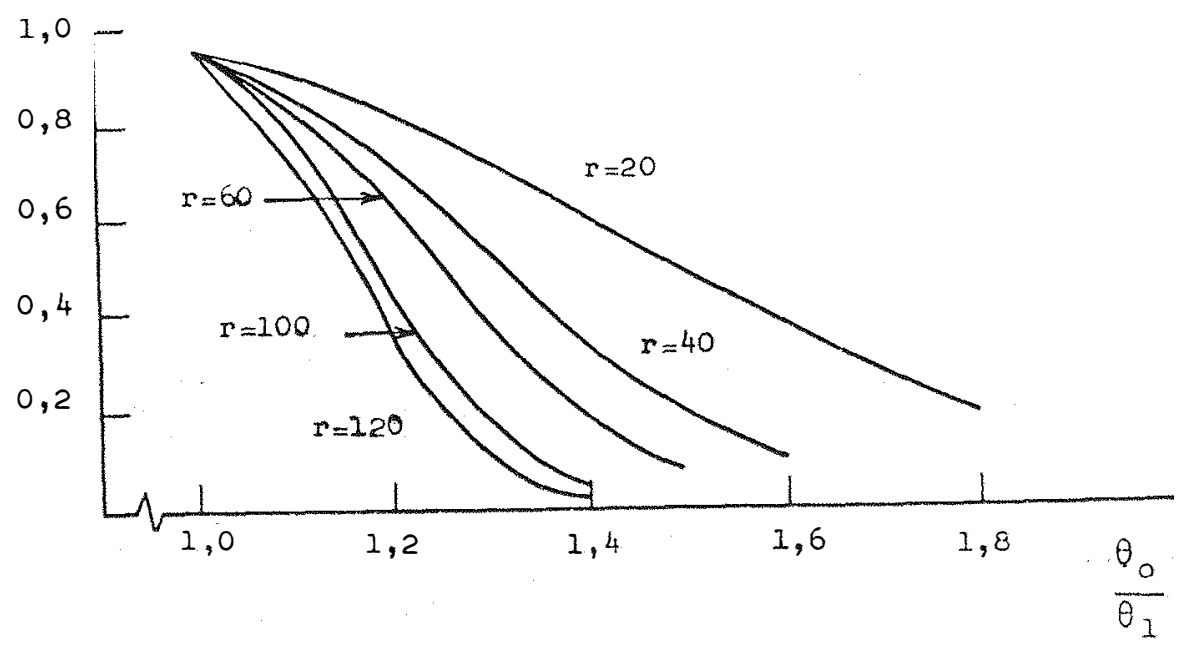

Teste para $H_{0}: \theta \geqslant \theta_{0}$ versus $H_{1}: \theta<\theta_{0} ; \alpha=0,05$ 
Figuras - continuação

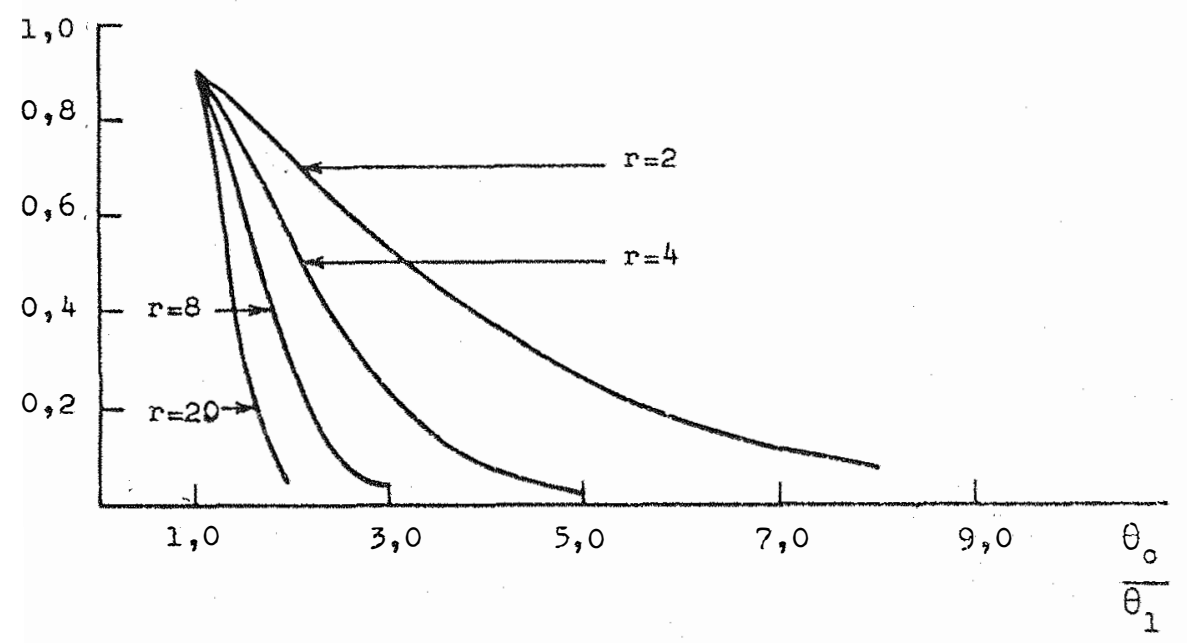

Teste para $H_{0}: \theta \geqslant \theta_{0}$ versus $H_{I}: \theta<\theta_{0} ; \alpha=0,10$

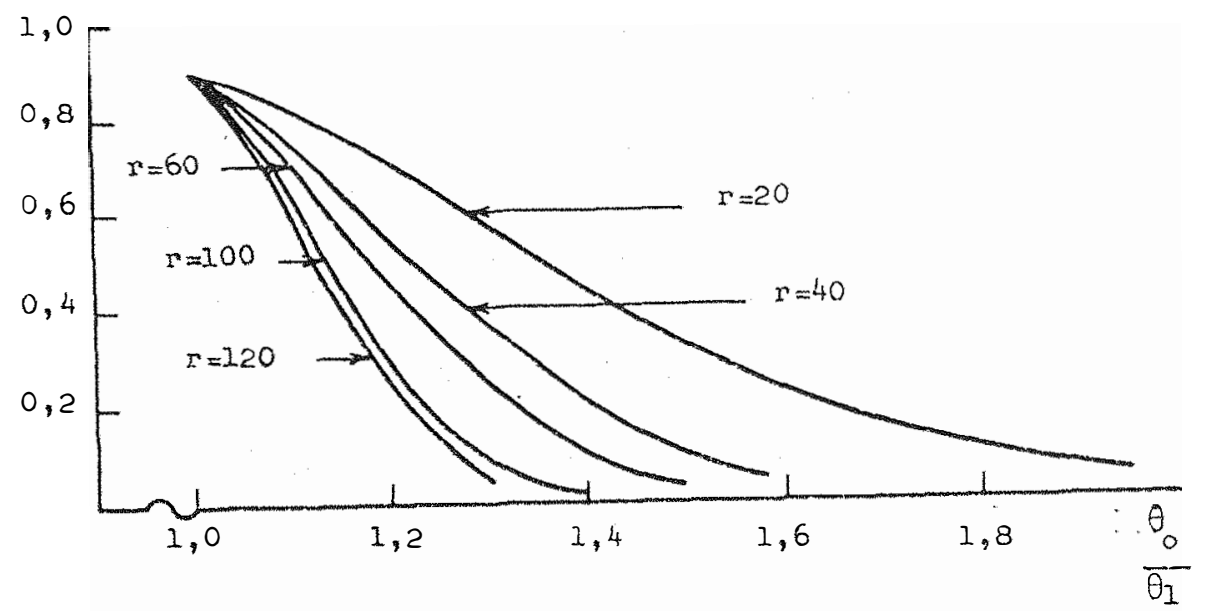

Teste para $H_{0}: \theta \geqslant \theta_{0}$ versus $H_{1}: \theta<\theta_{0} ; \alpha=0,10$ 
Figuras - continuação

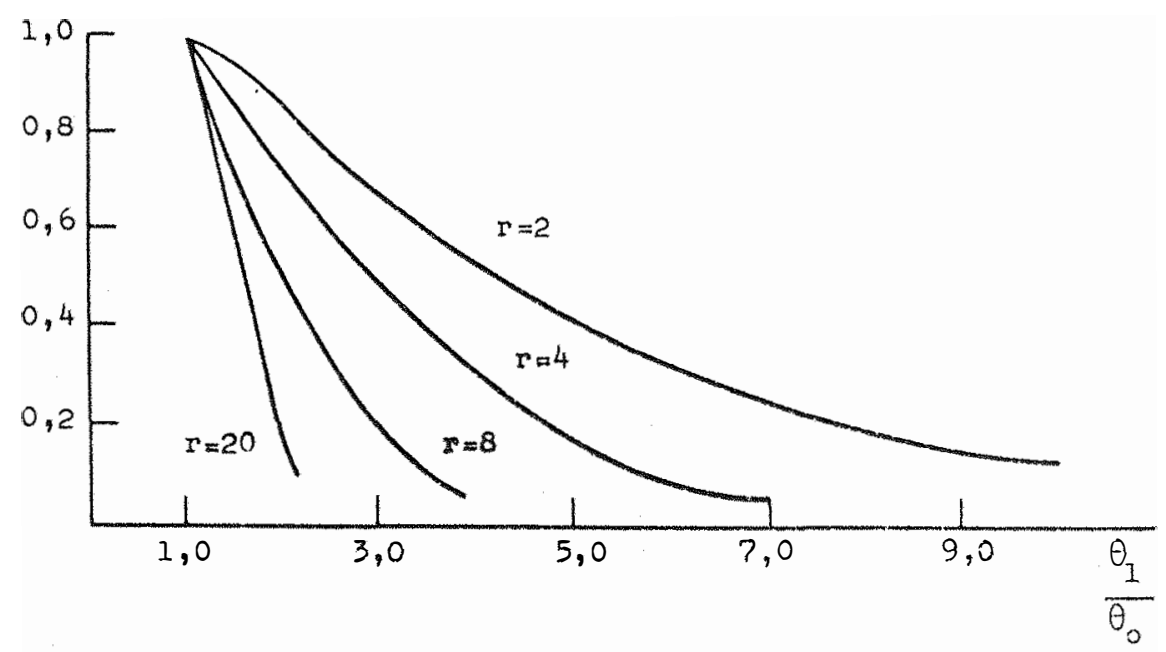

Teste para $H_{0}: \theta \leqslant \theta_{0}$ versus $H_{1}: \theta>\theta_{0} ; \alpha=0,01$

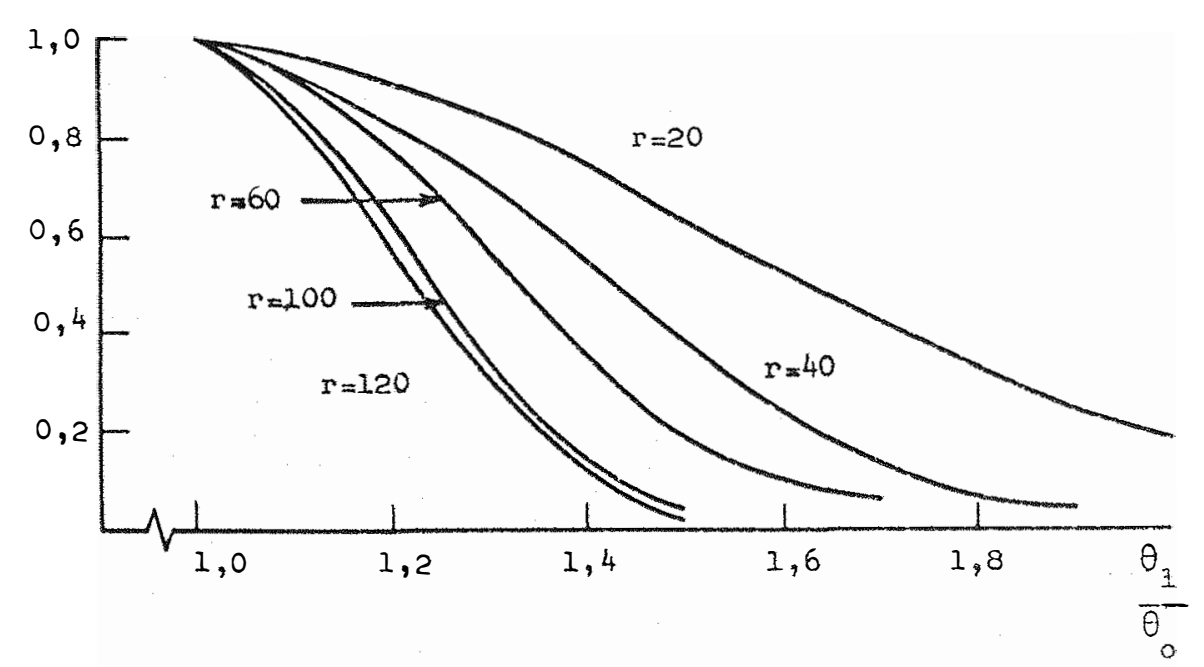

Teste para $\mathrm{H}_{0}: \theta \leqslant \theta_{0}$ versus $\mathrm{H}_{1}: \theta>\theta_{0} ; \alpha=0,01$ 
Figuras - continuaçäo

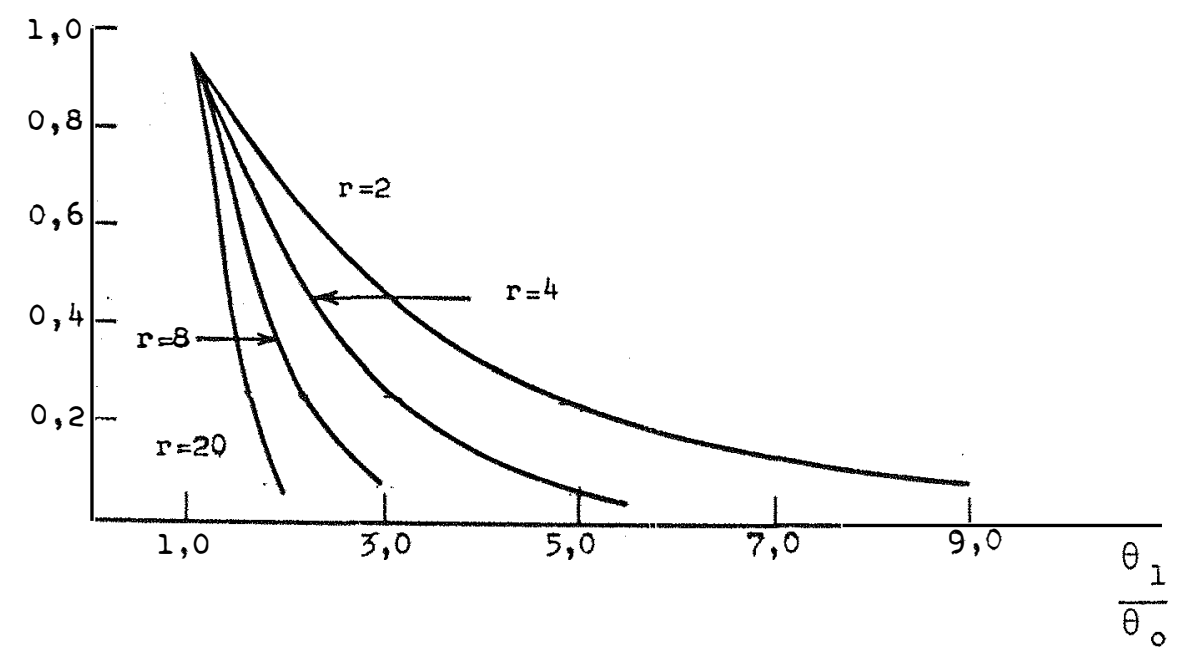

Teste para $H_{0}: \theta \leqslant \theta_{0}$ versus $H_{1}: \theta>\theta_{0} ; \alpha=0,05$

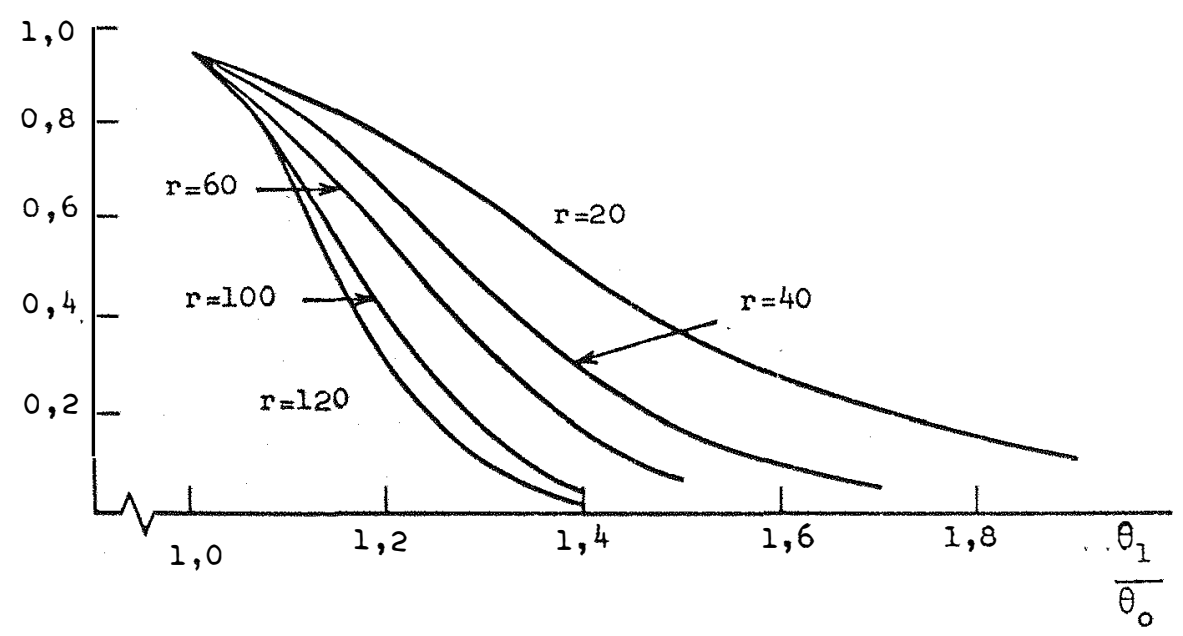

Teste para $H_{0}: \theta \leqslant \theta_{0}$ versus $H_{1}: \theta>\theta_{0} ; \alpha=0,05$ 
Figuras - continuação

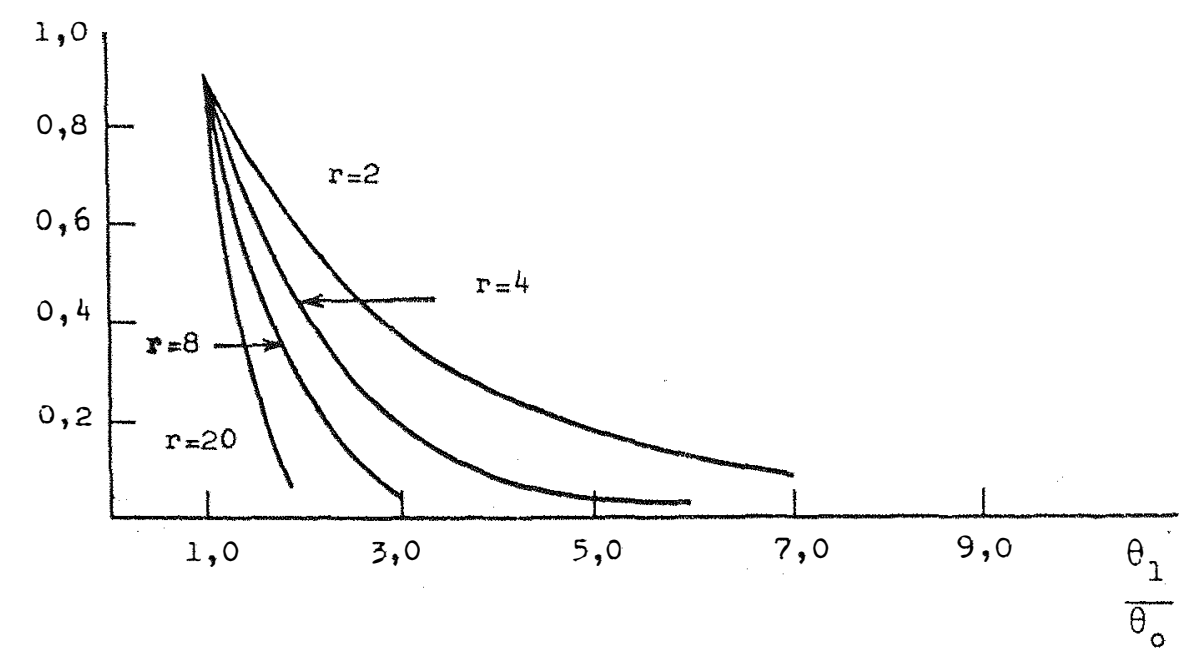

Teste para $H_{0}: \theta \leqslant \theta_{0}$ versus $H_{1}: \theta>\theta_{0} ; \alpha=0,10$

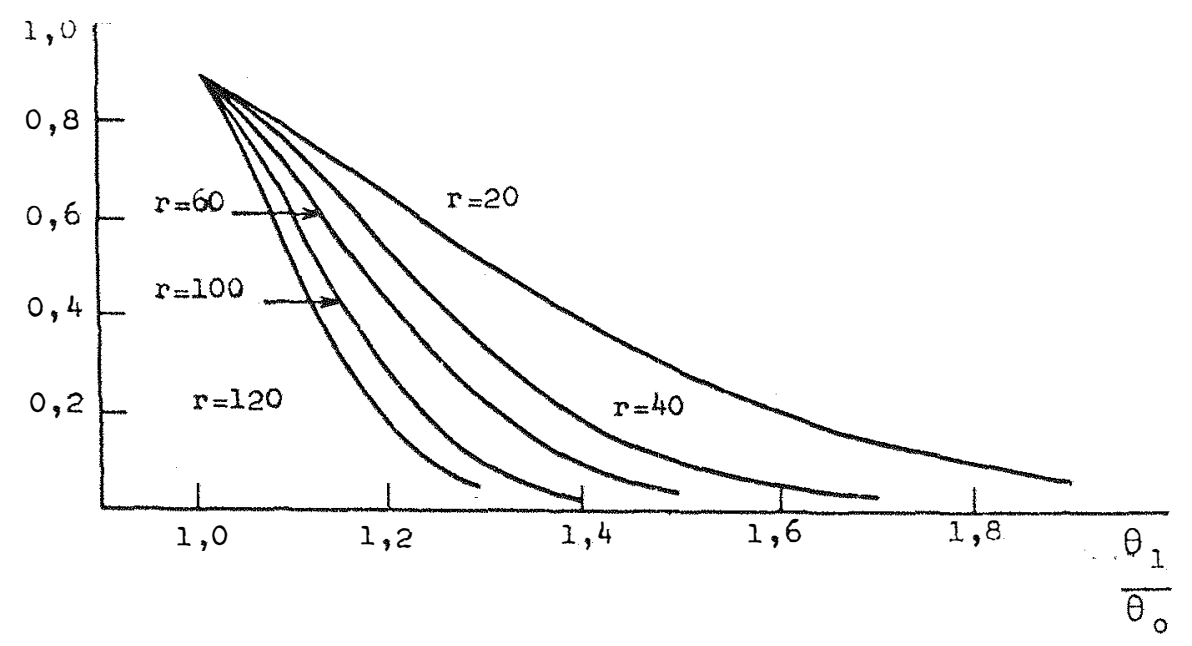

Teste para $H_{0}: \theta \leqslant \theta_{0}$ versus $H_{1}: \theta>\theta_{0} ; \alpha=0,10$ 
Tabela 1 - Valores de $c / \theta_{0}$ para o teste $H_{0}: \theta \geqslant \theta_{0}$ versus $H_{1}: \theta<\theta_{0}$. Censura tipo II.

\begin{tabular}{|c|c|c|c|c|c|}
\hline \multirow[b]{2}{*}{$\mathrm{r}$} & \multicolumn{5}{|c|}{$\alpha$} \\
\hline & 0,01 & 0,05 & 0,10 & 0,20 & 0,50 \\
\hline 1 & 0,010 & 0,052 & 0,106 & 0,288 & 0,693 \\
\hline 2 & 0,074 & 0,178 & 0,266 & 0,418 & 0,839 \\
\hline 3 & 0,145 & 0,272 & 0,367 & 0,576 & 0,891 \\
\hline 4 & 0,206 & 0,342 & 0,436 & 0,634 & 0,918 \\
\hline 5 & 0,256 & 0,349 & 0,487 & 0,674 & 0,934 \\
\hline 6 & 0,298 & 0,436 & 0,525 & 0,703 & 0,945 \\
\hline 7 & 0,333 & 0,469 & 0,556 & 0,726 & 0,953 \\
\hline 8 & 0,363 & 0,498 & 0,582 & 0,744 & 0,959 \\
\hline 9 & 0,390 & 0,522 & 0,604 & 0,760 & 0,963 \\
\hline 10 & 0,413 & 0,543 & 0,622 & 0,773 & 0,967 \\
\hline 15 & 0,498 & 0,616 & 0,687 & 0,816 & 0,978 \\
\hline 20 & 0,554 & 0,663 & 0,726 & 0,842 & 0,983 \\
\hline 25 & 0,594 & 0,665 & 0,754 & 0,859 & 0,987 \\
\hline 30 & 0,625 & 0,720 & 0,774 & 0,872 & 0,989 \\
\hline 35 & 0,649 & 0,739 & 0,790 & 0,881 & 0,990 \\
\hline 40 & 0,669 & 0,755 & 0,804 & 0,889 & 0,991 \\
\hline 45 & 0,687 & 0,768 & 0,814 & 0,896 & 0,992 \\
\hline 50 & 0,701 & 0,779 & 0,824 & 0,901 & 0,993 \\
\hline
\end{tabular}


Tabela 2 - Valores de $C / \theta_{0}$ para o teste $H_{0}: \theta \leqslant \theta_{0}$ versus $H_{1}: \theta>\theta_{1}$. Censura tipo II.

\begin{tabular}{|c|c|c|c|c|c|}
\hline \multirow[b]{2}{*}{$\mathrm{r}$} & \multicolumn{5}{|c|}{$\alpha$} \\
\hline & 0,01 & 0,05 & 0,10 & 0,25 & 0,50 \\
\hline 1 & 4,605 & 2,996 & 2,303 & 1,387 & 0,693 \\
\hline 2 & 3,319 & 2,372 & 1,945 & 1,346 & 0,839 \\
\hline 3 & 2,802 & 2,099 & 1,774 & 1,307 & 0,891 \\
\hline 4 & 2,511 & 1,938 & 1,670 & 1,277 & 0,918 \\
\hline 5 & 2,321 & 1,831 & 1,599 & 1,255 & 0,934 \\
\hline 6 & 2,185 & 1,827 & 1,546 & 1,237 & 0,945 \\
\hline 7 & 2,082 & 1,692 & 1,505 & 1,223 & 0,953 \\
\hline 8 & 2,000 & 1,644 & 1,471 & 1,211 & 0,959 \\
\hline 9 & 1,934 & 1,604 & 1,444 & 1,200 & 0,963 \\
\hline 10 & 1,878 & 1,571 & 1,421 & 1,191 & 0,967 \\
\hline 15 & 1,696 & 1,459 & 1,342 & 1,160 & 0,978 \\
\hline 20 & 1,593 & 1,394 & 1,295 & 1,140 & 0,983 \\
\hline 25 & 1,523 & 1,350 & 1,263 & 1,127 & 0,987 \\
\hline 30 & 1,472 & 1,318 & 1,240 & 1,116 & 0,988 \\
\hline 35 & 1,435 & 1,293 & 1,221 & 1,108 & 0,990 \\
\hline 40 & 1,404 & 1,273 & 1,207 & 1,102 & 0,991 \\
\hline 45 & 1,379 & 1,257 & 1,195 & 1,096 & 0,992 \\
\hline 50 & 1,358 & 1,243 & 1,185 & 1,091 & 0,993 \\
\hline
\end{tabular}


Tabela 3 - Fornece $x_{2 r_{0}, 1-\alpha}^{2} / X_{2 r_{0}}^{2}, B$ para se determinar $r_{0}$ em um teste do tipo $\mathrm{H}_{0}: \theta \geqslant \theta_{0}$ versus $\mathrm{H}_{1}: \theta \leqslant \theta_{0}$. Censura tipo II.

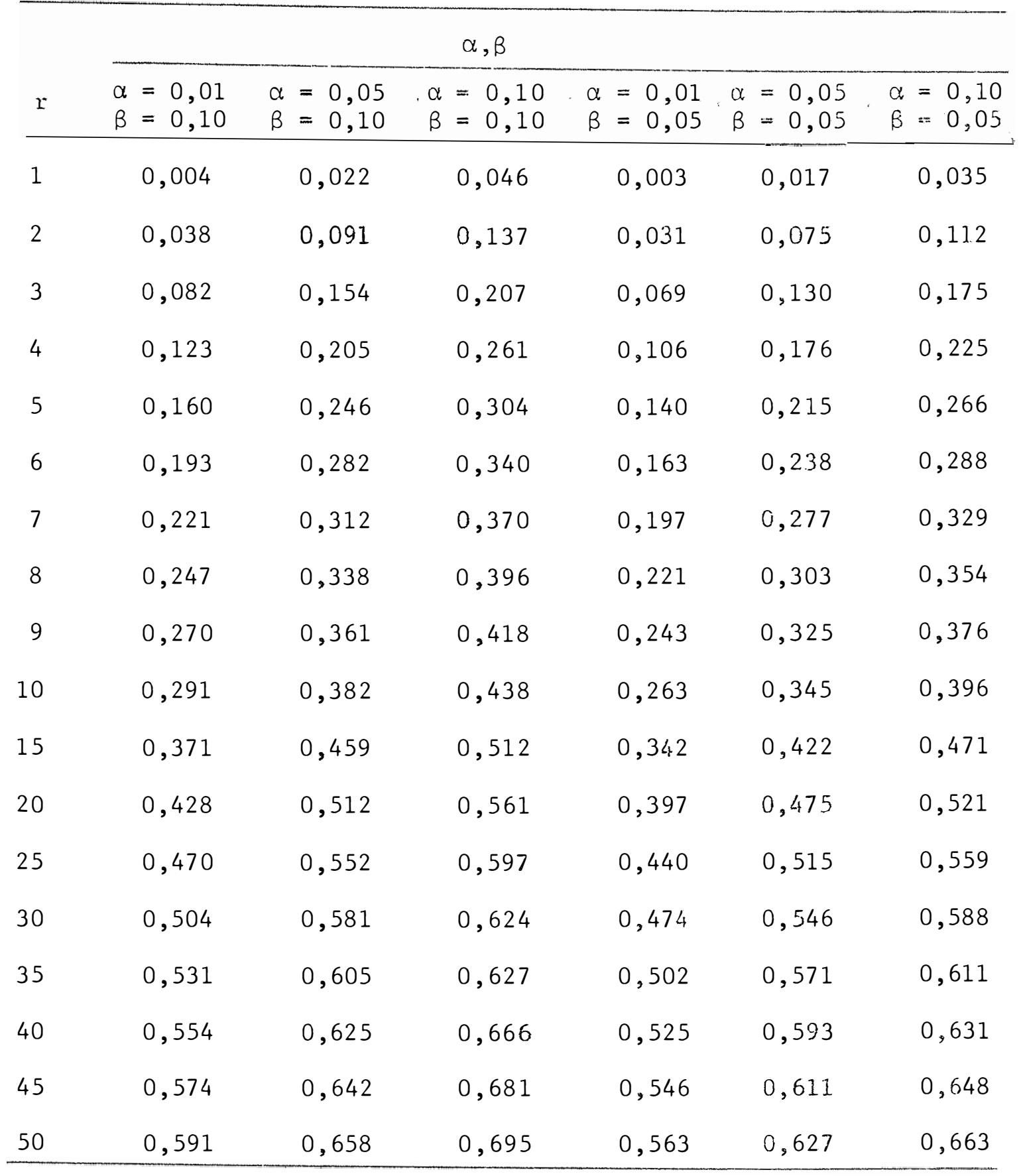


Tabela 4 - Fornece $x_{2 r_{0}, \alpha}^{2} / x_{2 r_{0}, 1-\beta}^{2}$ para se determinar $r_{0}$ em um: teste do tipo $H_{0}: \theta \leqslant \theta_{0}$. versus $H_{1}: \theta>\theta_{0}$. Censura tipo II.

\begin{tabular}{|c|c|c|c|c|c|c|}
\hline \multirow[b]{2}{*}{$r$} & \multicolumn{6}{|c|}{$\alpha, \beta$} \\
\hline & $\begin{array}{l}\alpha=0,01 \\
\beta=0,10\end{array}$ & $\begin{array}{l}\alpha=0,05 \\
\beta=0,10\end{array}$ & $\begin{array}{l}\alpha=0,10 \\
\beta=0,10\end{array}$ & $\begin{array}{l}\alpha=0,01 \\
\beta=0,05\end{array}$ & $\begin{array}{l}\alpha=0,05 \\
\beta=0,05\end{array}$ & $\begin{array}{l}\alpha=0,10 \\
\beta=0,05\end{array}$ \\
\hline 1 & 13,649 & 28,393 & 21,825 & 89,417 & 58,165 & 44,709 \\
\hline 2 & 12,478 & 8,917 & 7,311 & 18,674 & 13,345 & 10,941 \\
\hline 3 & 7,628 & 5,713 & 4,830 & 10,283 & 7,702 & 6,511 \\
\hline 4 & 5,756 & 4,443 & 3,829 & 7,351 & 5,674 & 4,889 \\
\hline 5 & 4,771 & 3,763 & 3,286 & 5,891 & 4,645 & 4,058 \\
\hline 6 & 4,159 & 3,477 & 2,942 & 5,017 & 4,194 & 3,549 \\
\hline 7 & 3,741 & 3,040 & 2,704 & 4,435 & 3,604 & 3,206 \\
\hline 8 & 3,436 & 2,824 & 2,528 & 4,019 & 3,303 & 2,957 \\
\hline 9 & 3,203 & 2,657 & 2,392 & 3,707 & 3,074 & 2,768 \\
\hline 10 & 3,019 & 2,524 & 2,283 & 3,462 & 2,895 & 2,618 \\
\hline 15 & 2,471 & 2,125 & 1,954 & 2,752 & 2,367 & 2,177 \\
\hline 20 & 2,192 & 1,918 & 1,781 & 2,403 & 2,103 & 1,953 \\
\hline 25 & 2,020 & 1,720 & 1,675 & 2,191 & 1,942 & 1,816 \\
\hline 30 & 1,902 & 1,701 & 1,600 & 2,047 & 1,831 & 1,722 \\
\hline 35 & 1,815 & 1,636 & 1,545 & 1,941 & 1,750 & 1,653 \\
\hline 40 & 1,747 & 1,584 & 1,502 & 1,860 & 1,687 & 1,599 \\
\hline 45 & 1,693 & 1,543 & 1,467 & 1,796 & 1,637 & 1,556 \\
\hline 50 & 1,649 & 1,509 & 1,438 & 1,743 & 1,596 & 1,520 \\
\hline
\end{tabular}


Tabela 5 - Estatỉstica teste e regiões crîticas para os testes relativos a vida mẻdia. Censura tipo II.

\begin{tabular}{|c|c|c|c|}
\hline $\mathrm{H}_{\mathrm{O}}$ & $\begin{array}{c}\text { Estatistica } \\
\text { teste }\end{array}$ & $\mathrm{H}_{1}$ & $\begin{array}{c}\text { Região Critica } \\
(\text { R.C.) }\end{array}$ \\
\hline$\theta \leqslant \theta_{0}$ & $x^{2}=\frac{2 r \hat{\theta}}{\theta_{0}}$ & $\theta>\theta_{0}$ & $x^{2}>x_{2 r, a}^{2}$ \\
\hline$\theta \geqslant \theta_{0}$ & $x^{2}=\frac{2 r \hat{\theta}}{\theta_{0}}$ & $\theta<\theta_{0}$ & $x^{2}<x_{2 r, 1-\alpha}^{2}$ \\
\hline$\theta=\theta_{0}$ & $x^{2}=\frac{2 r \hat{\theta}}{\theta_{0}}$ & $\theta \neq \theta_{0}$ & $\begin{array}{l}x^{2}<x_{2 x, 1-\alpha / 2}^{2} \\
x^{2}>x_{2 x, \alpha / 2}^{2} \\
r \text { grande }\end{array}$ \\
\hline
\end{tabular}




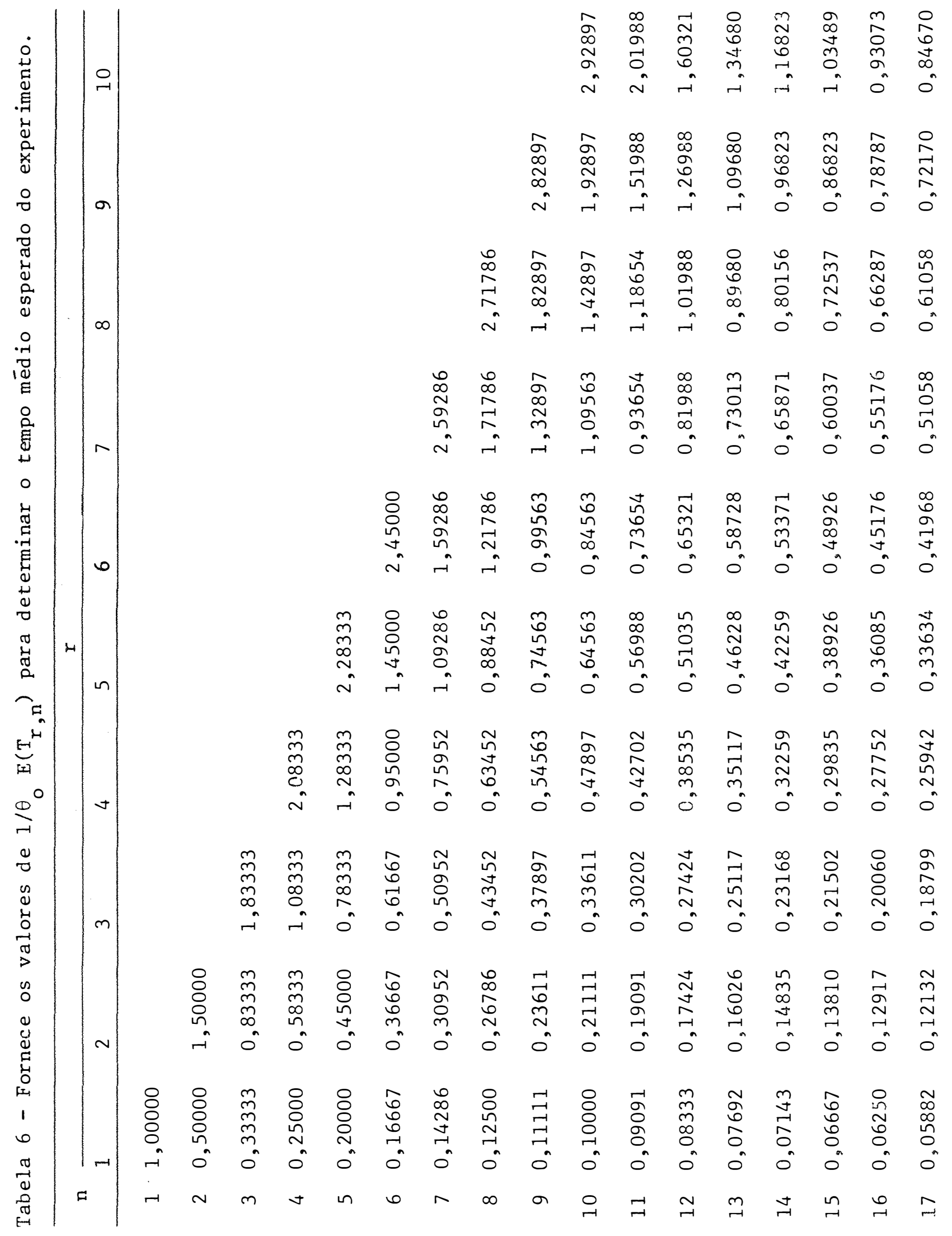




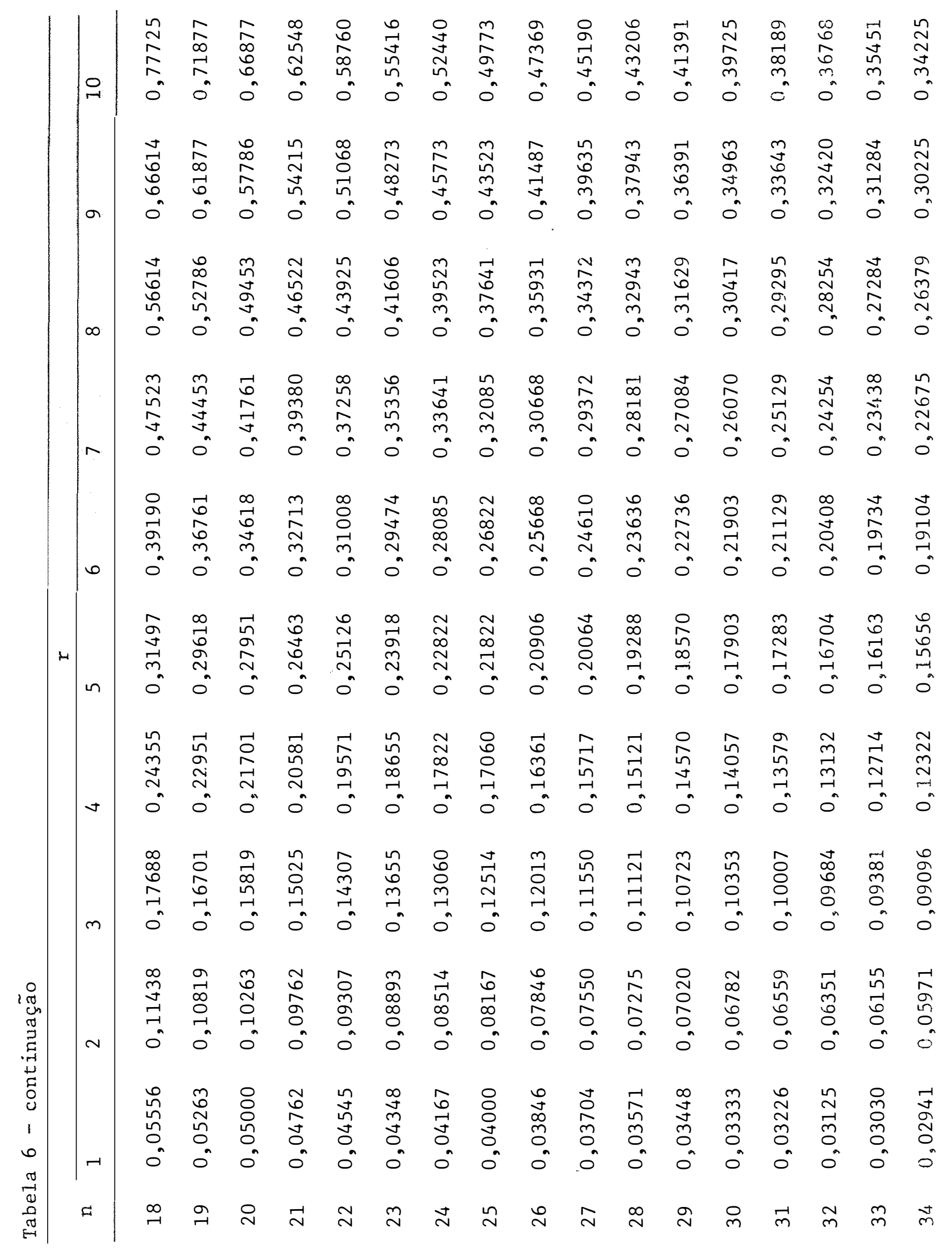




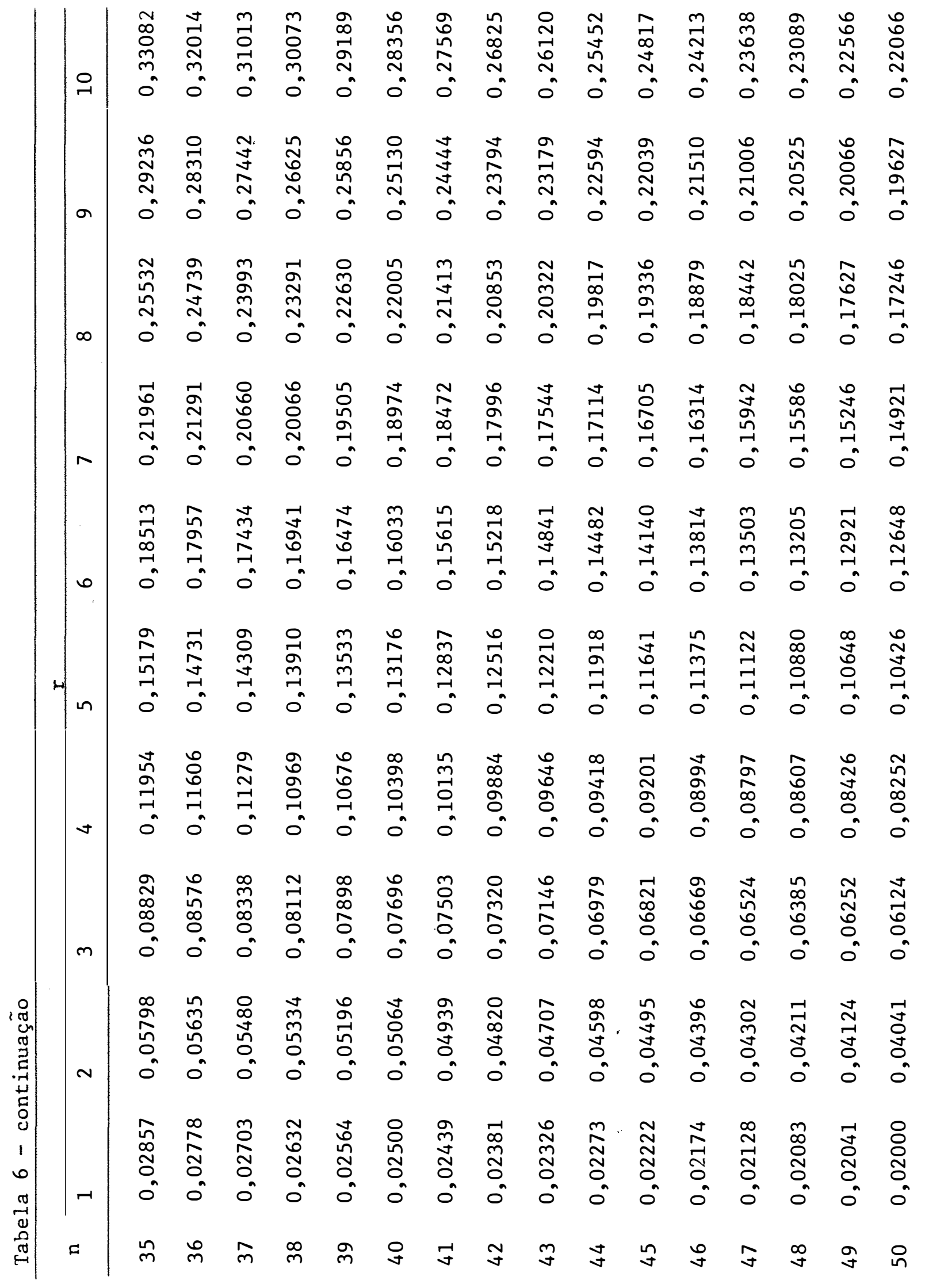


111.

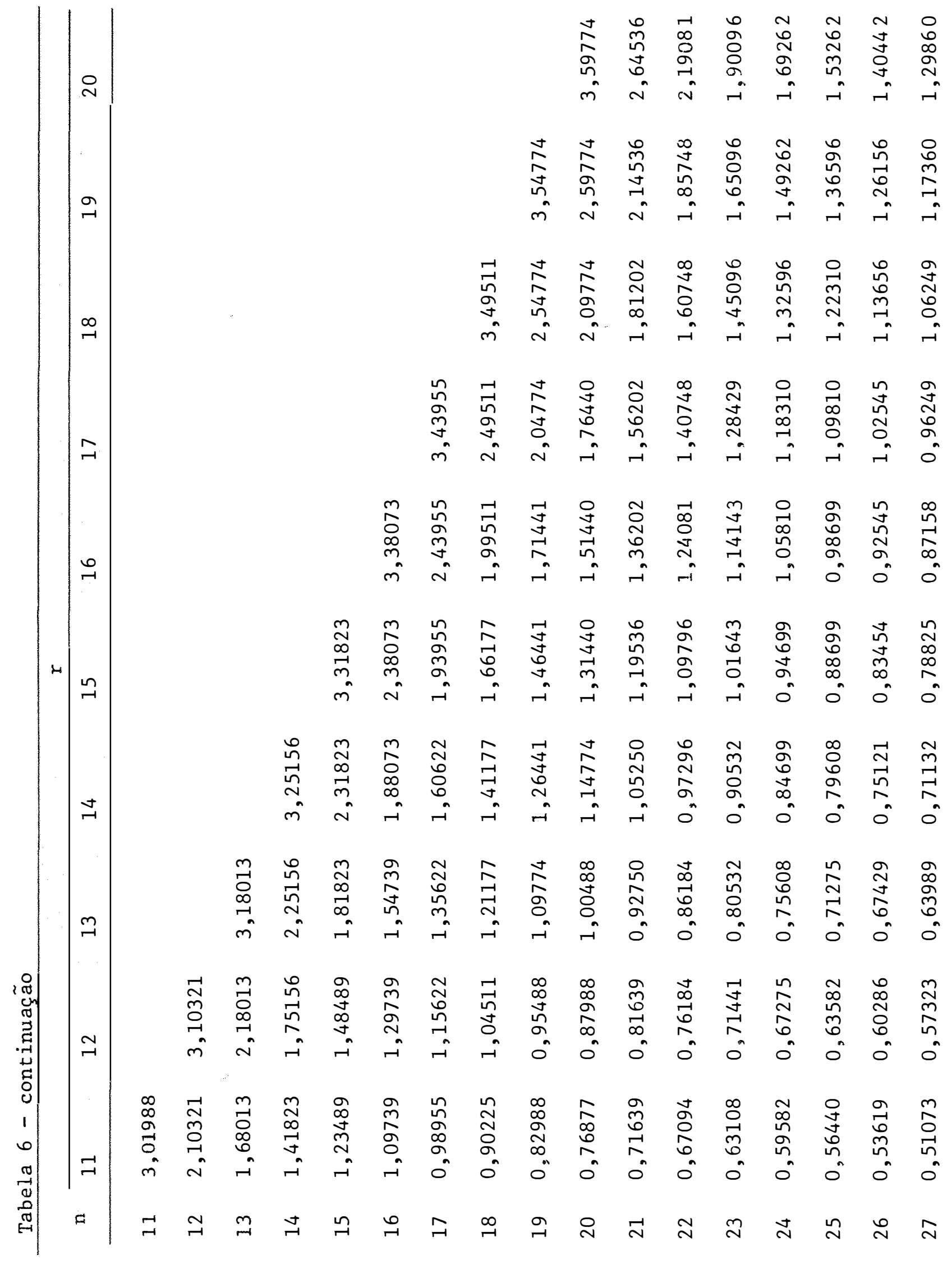




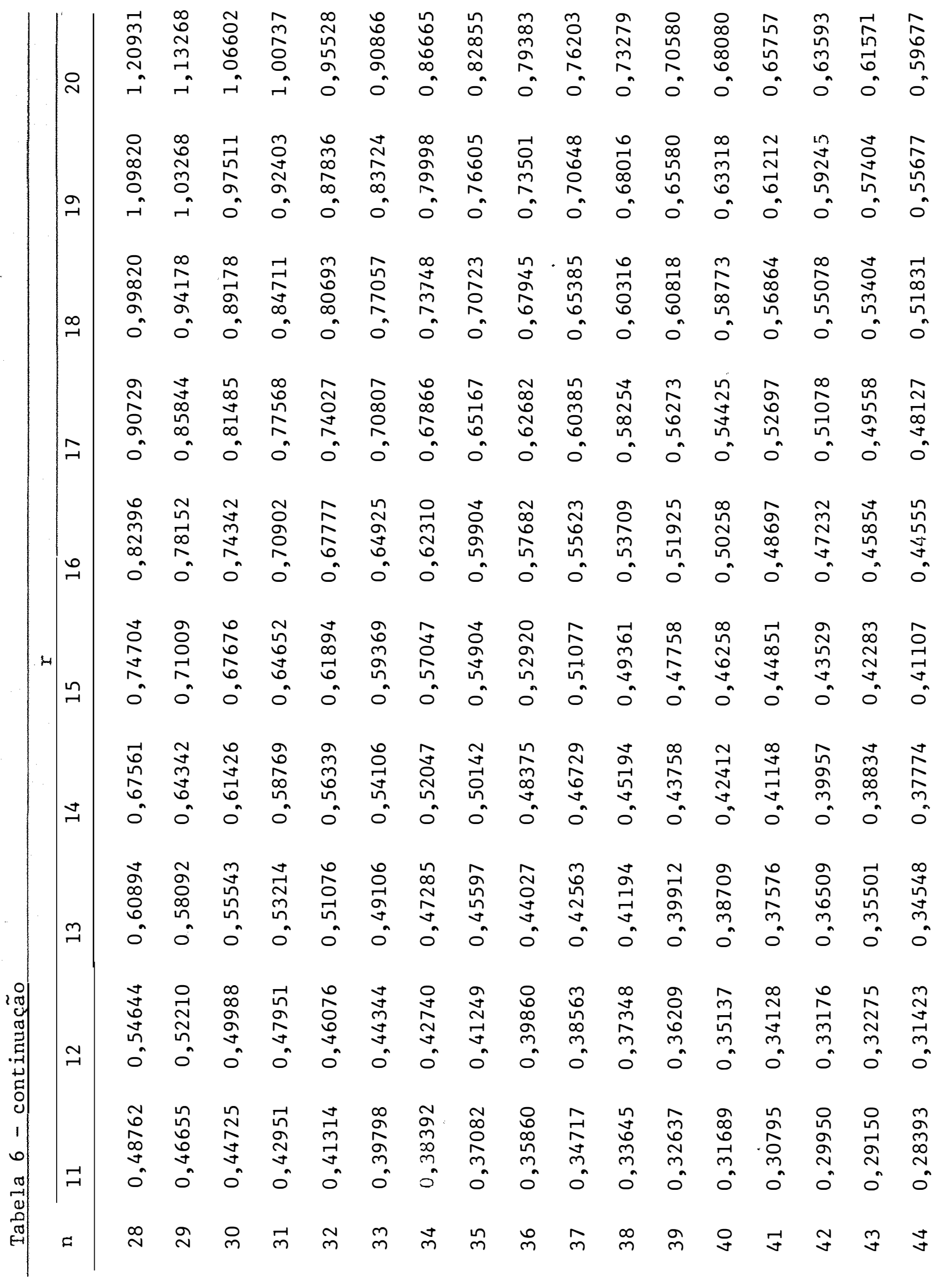


113.

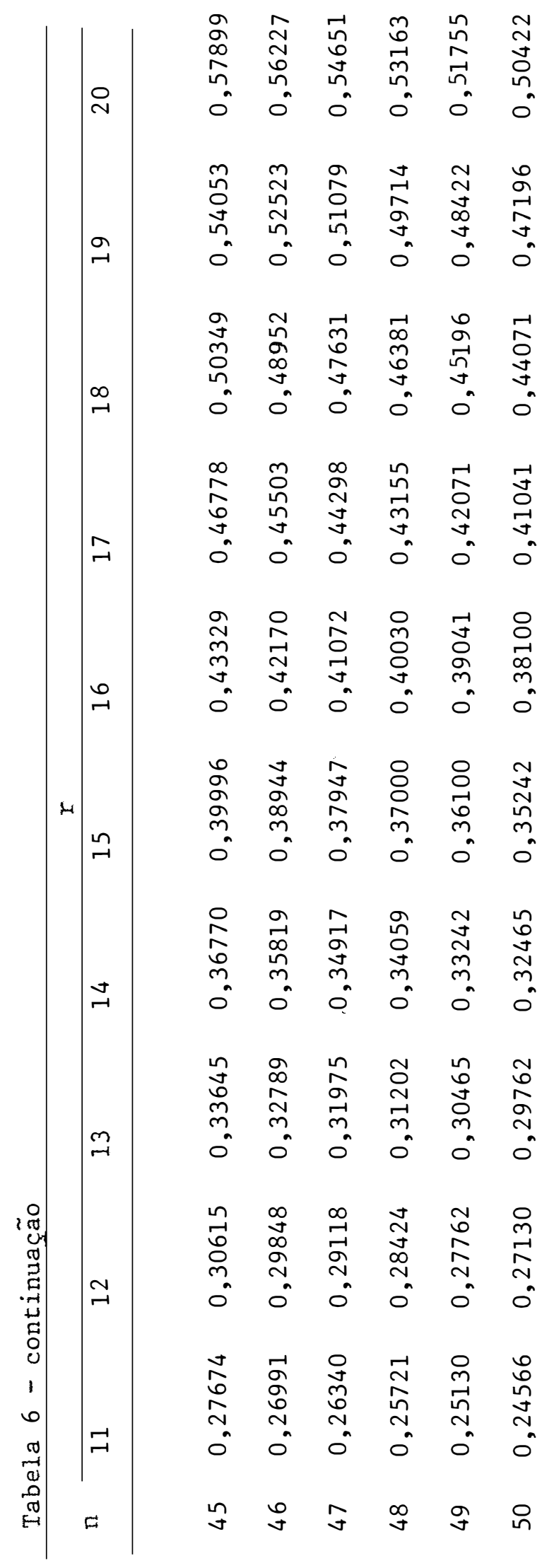




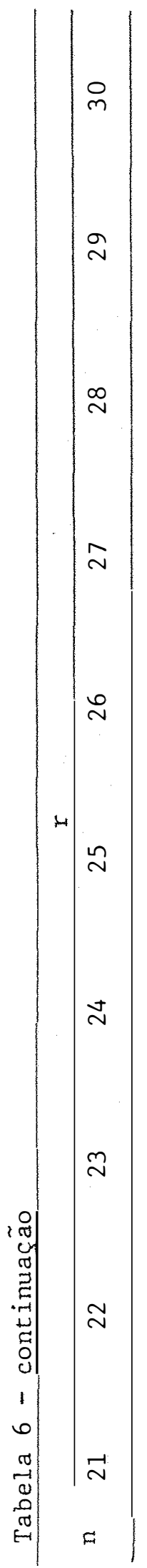

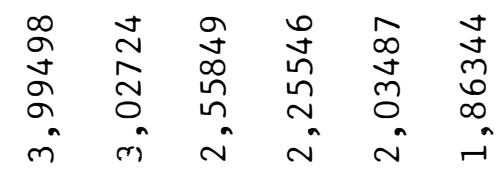

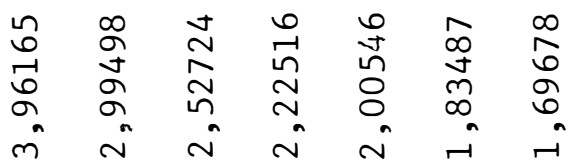

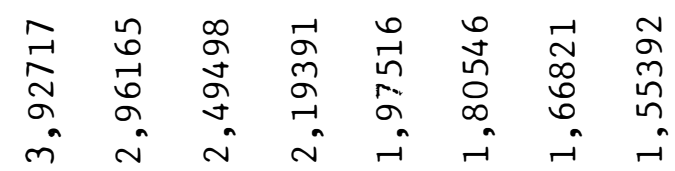

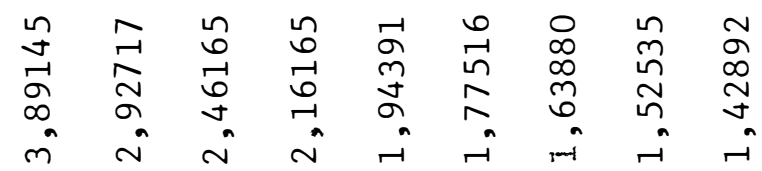

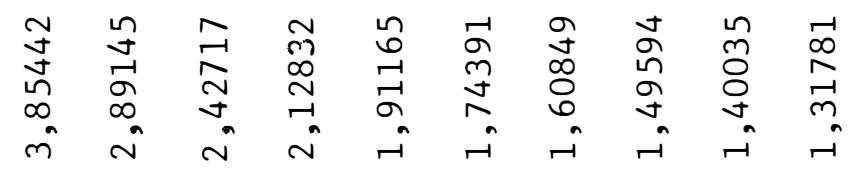

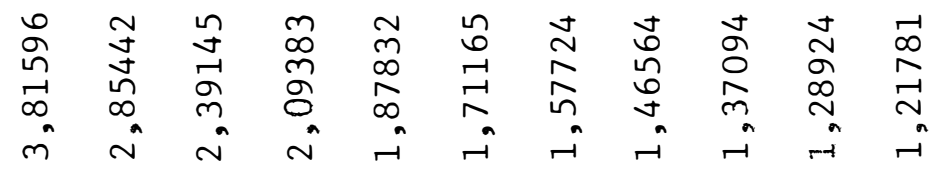

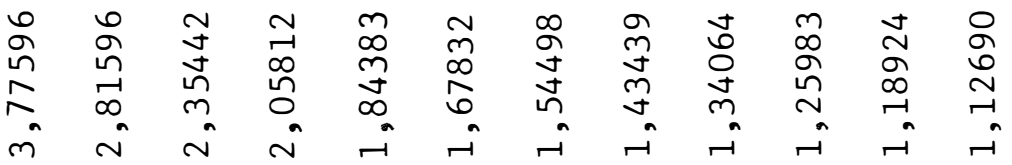

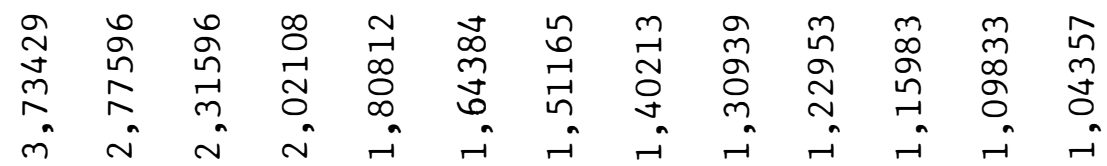

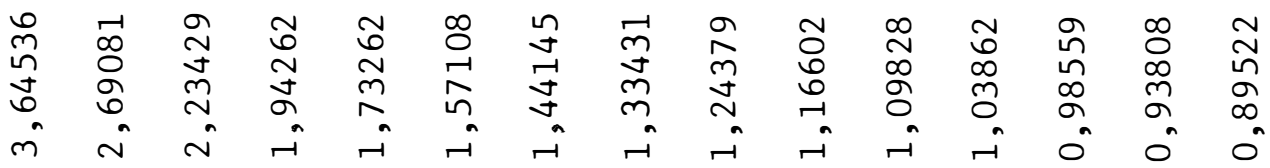

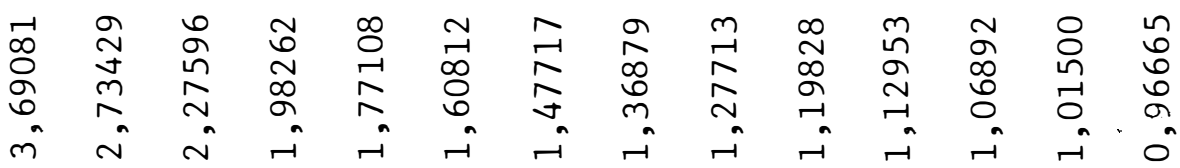

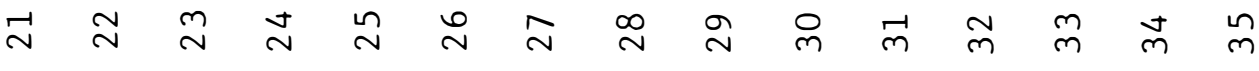




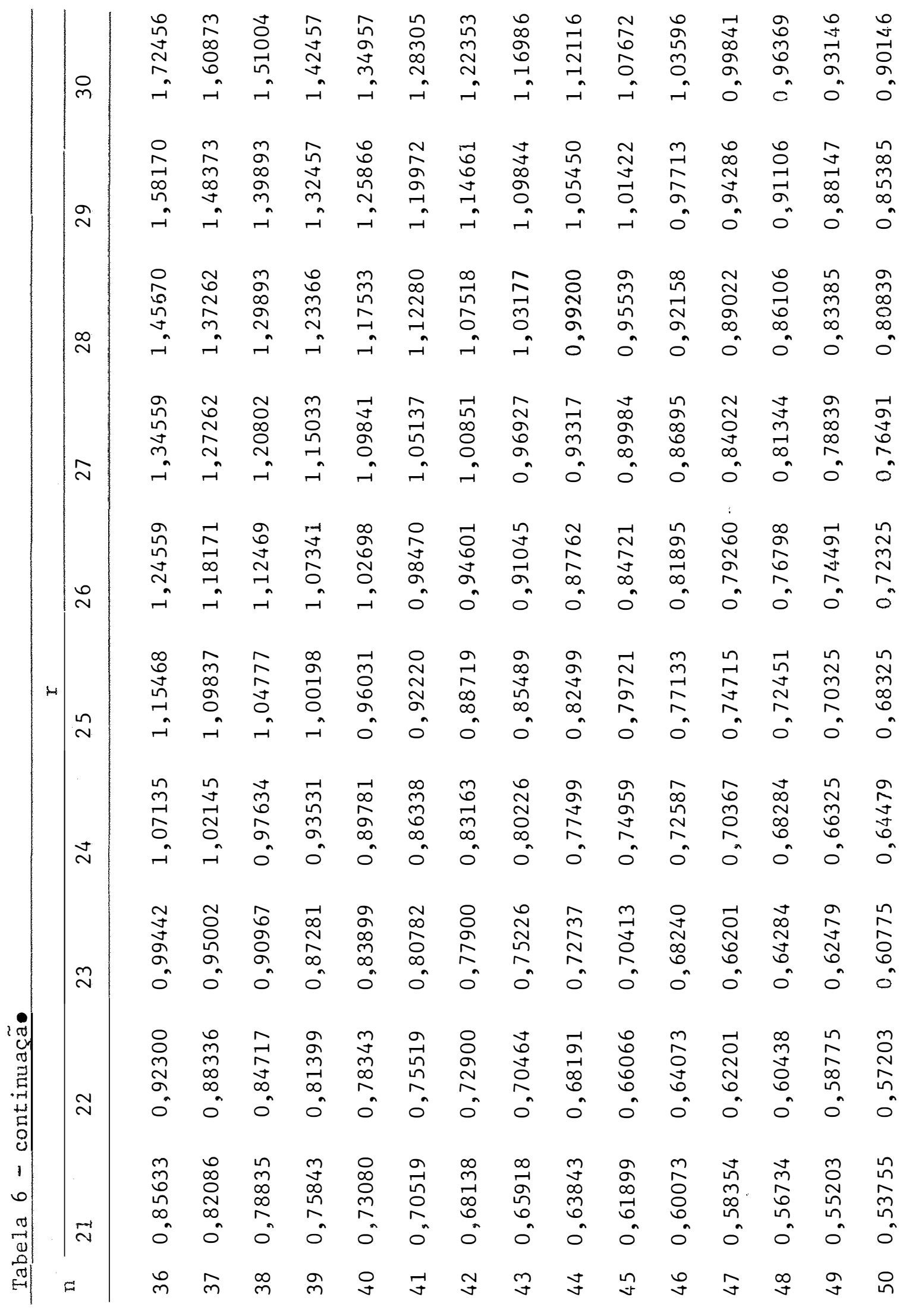


116.

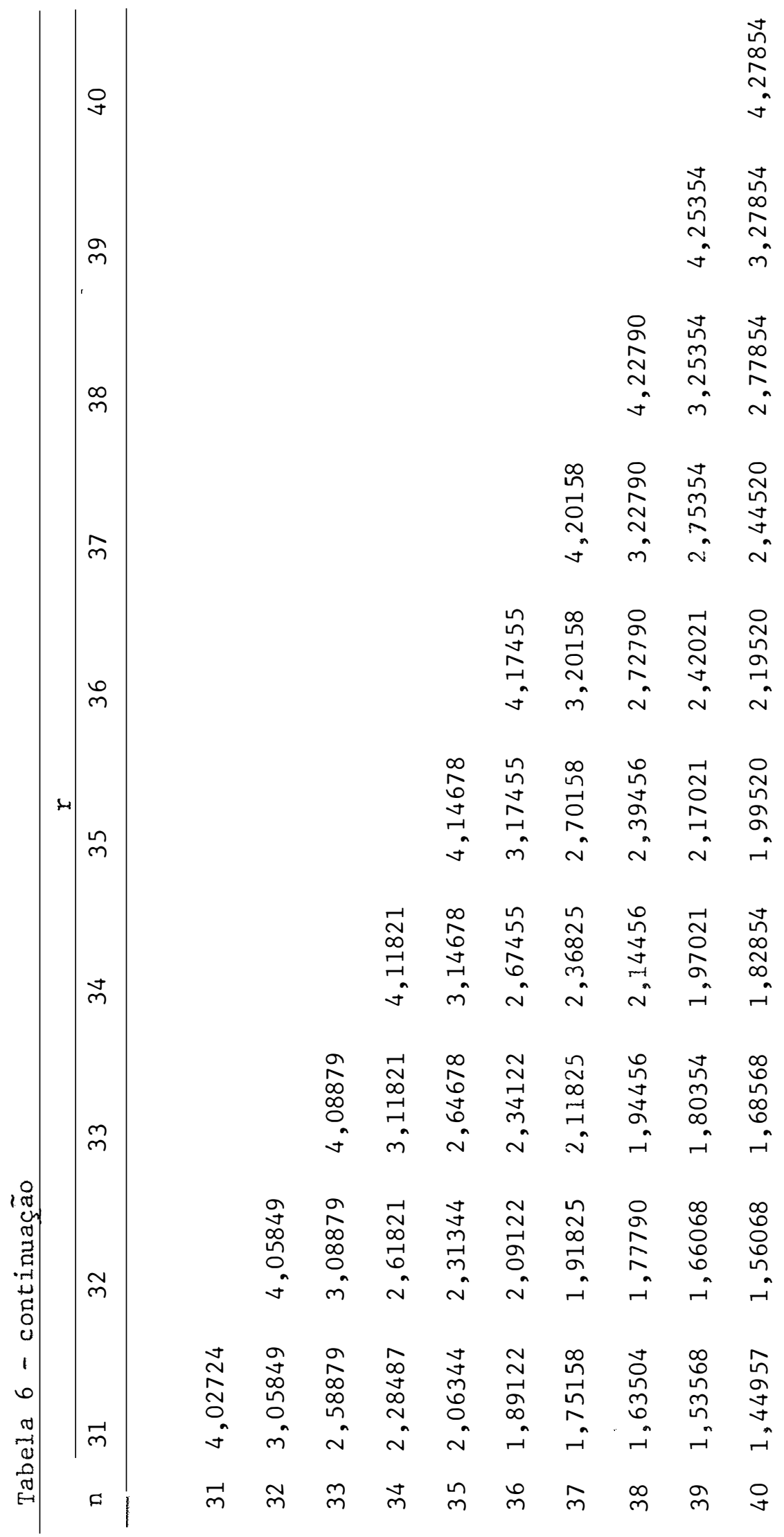




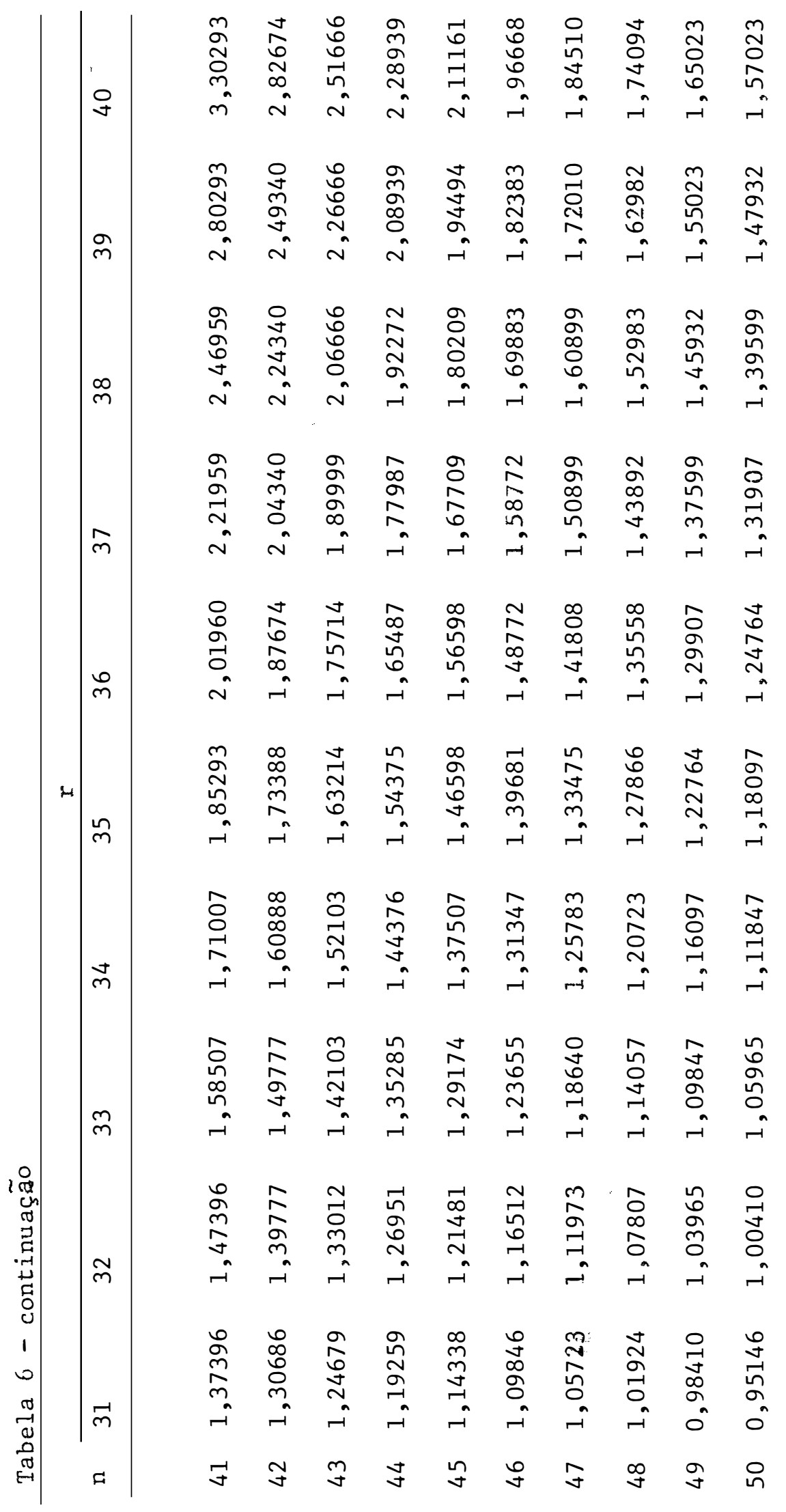


118.

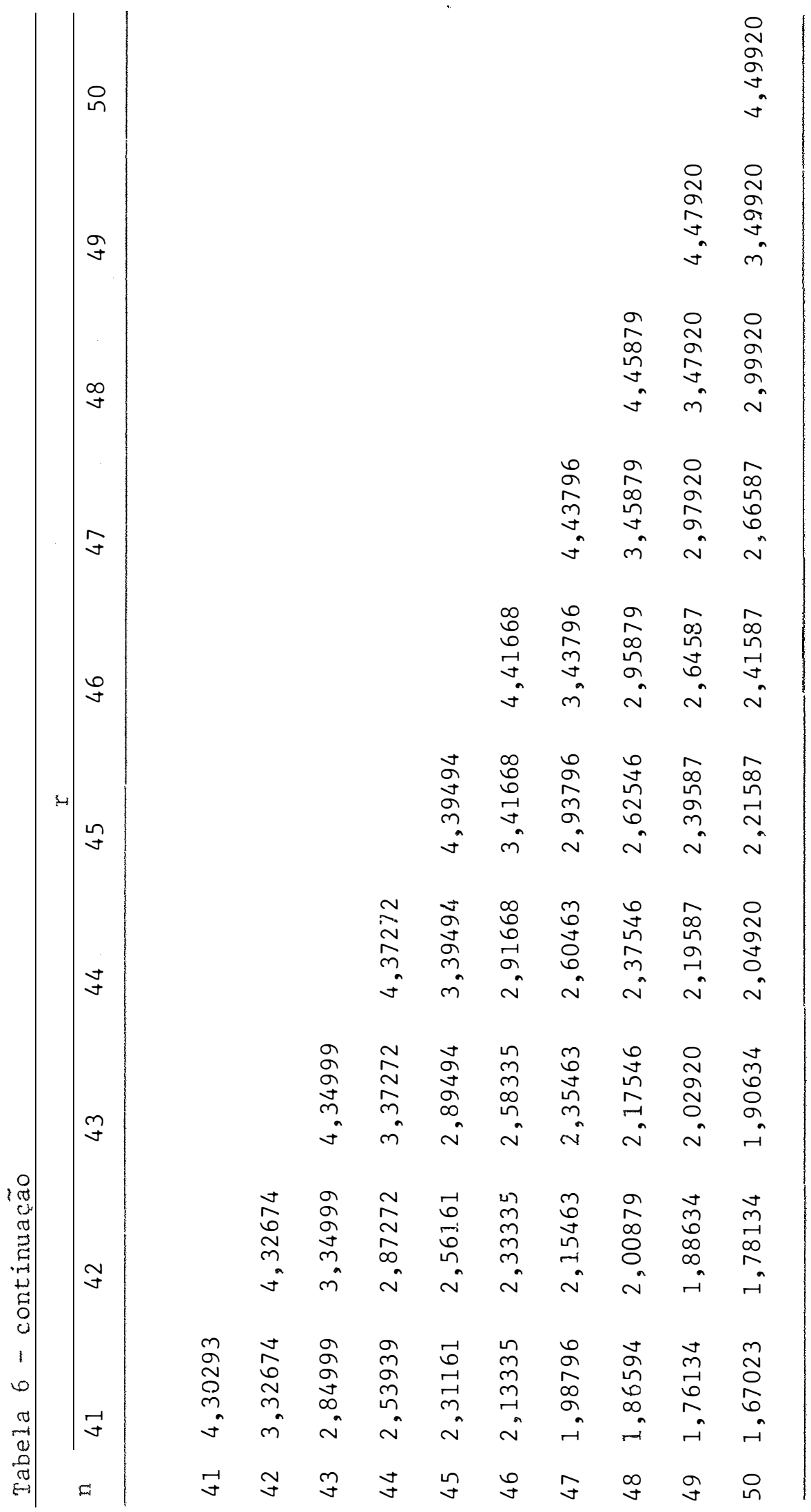


119.

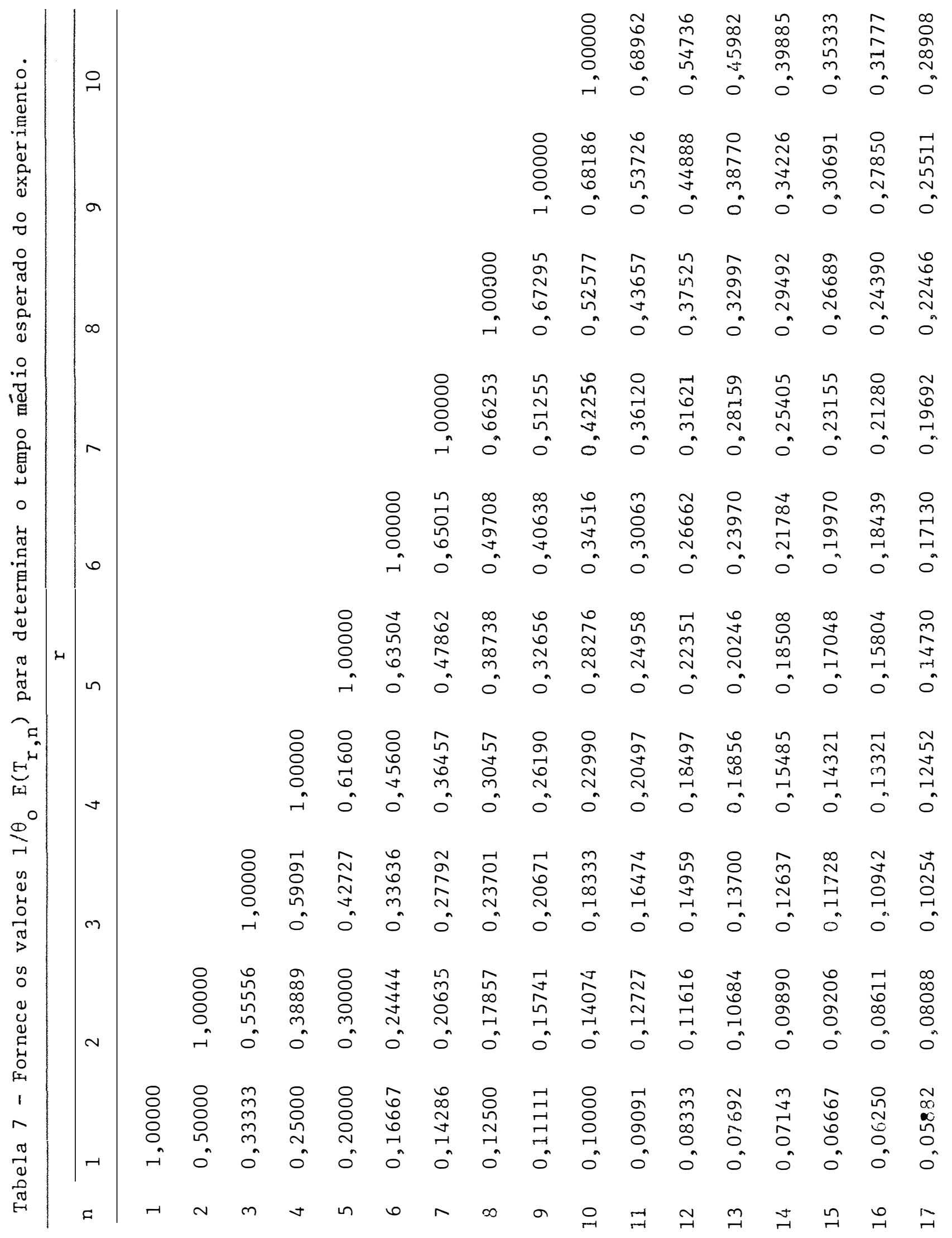




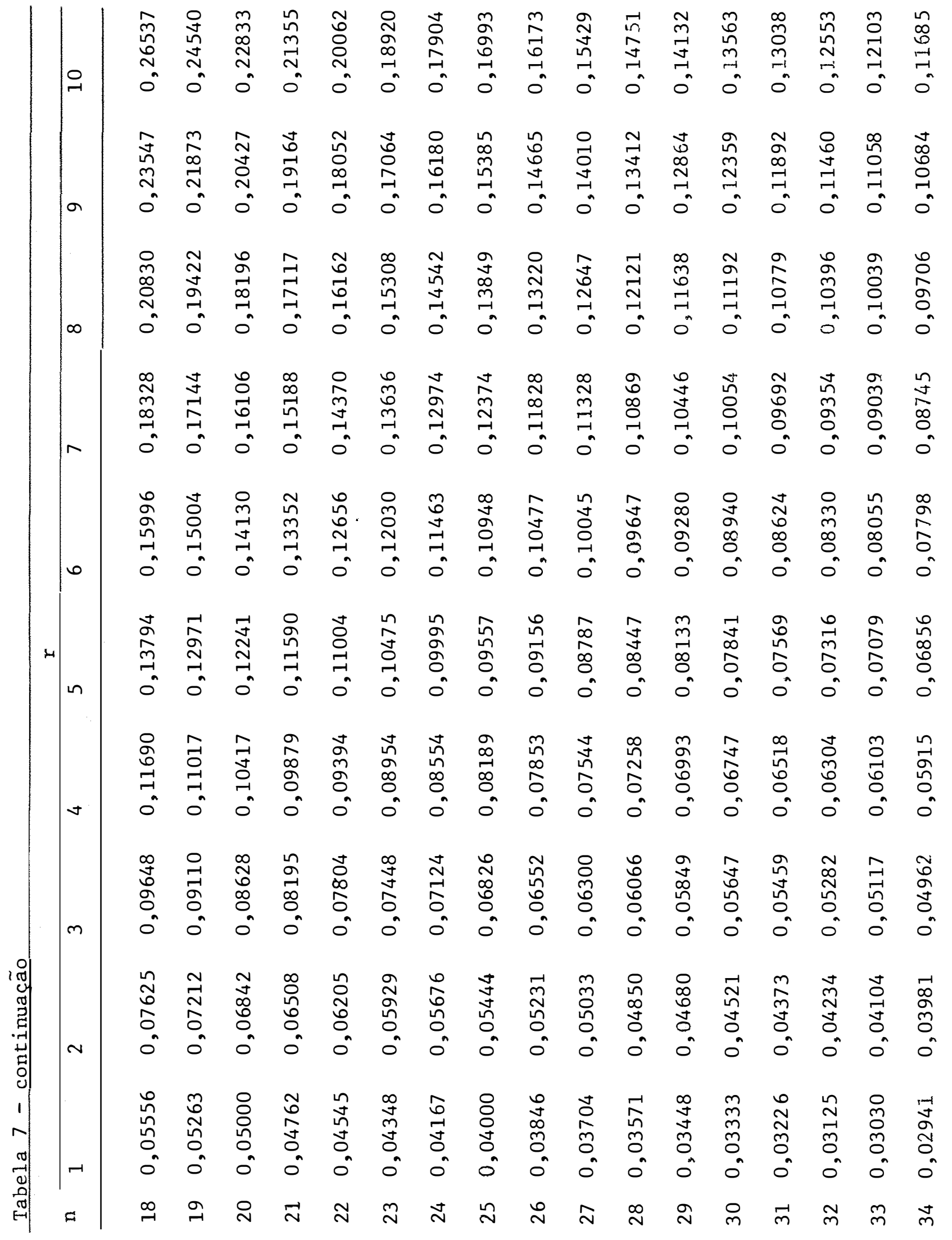




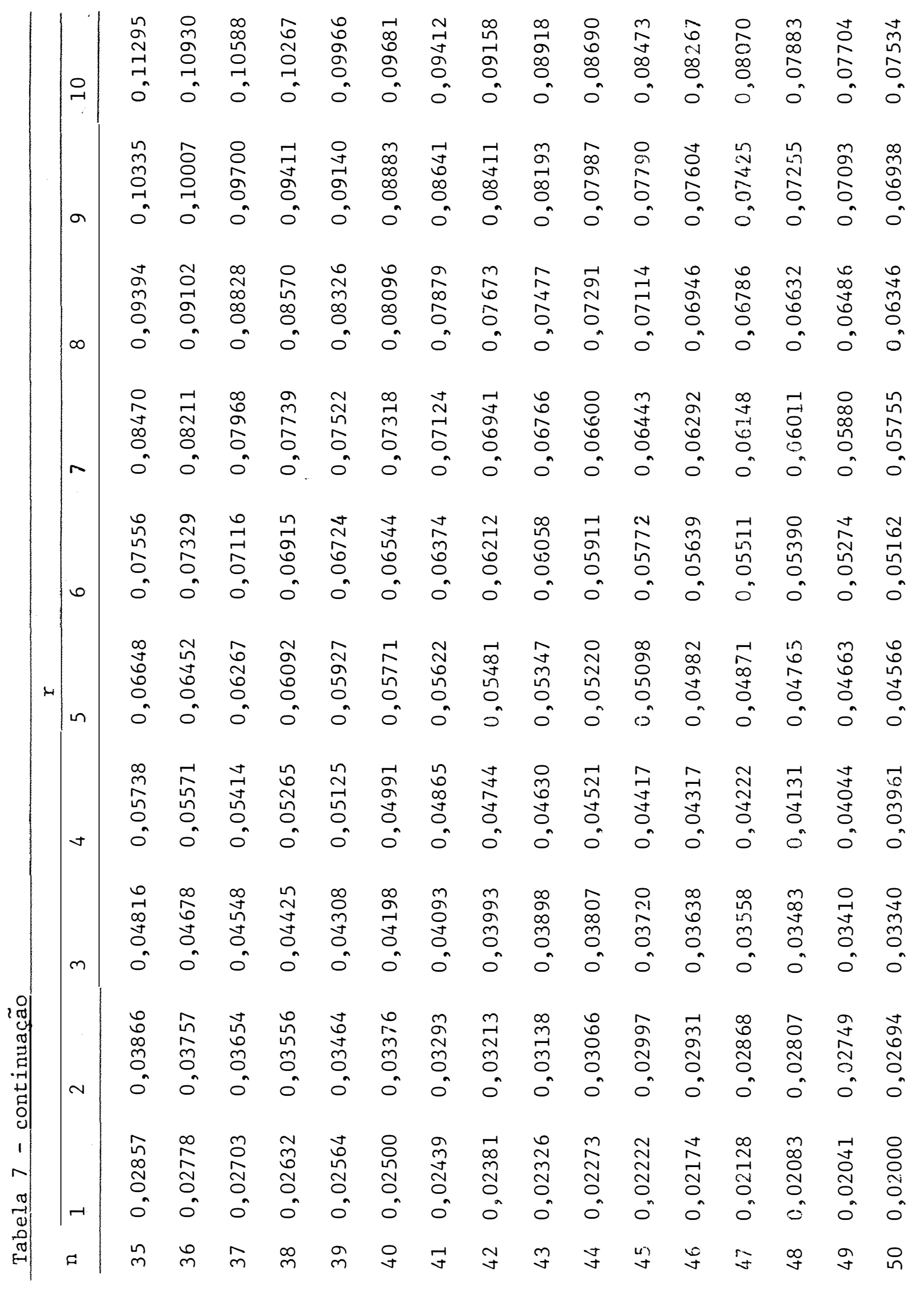


122.

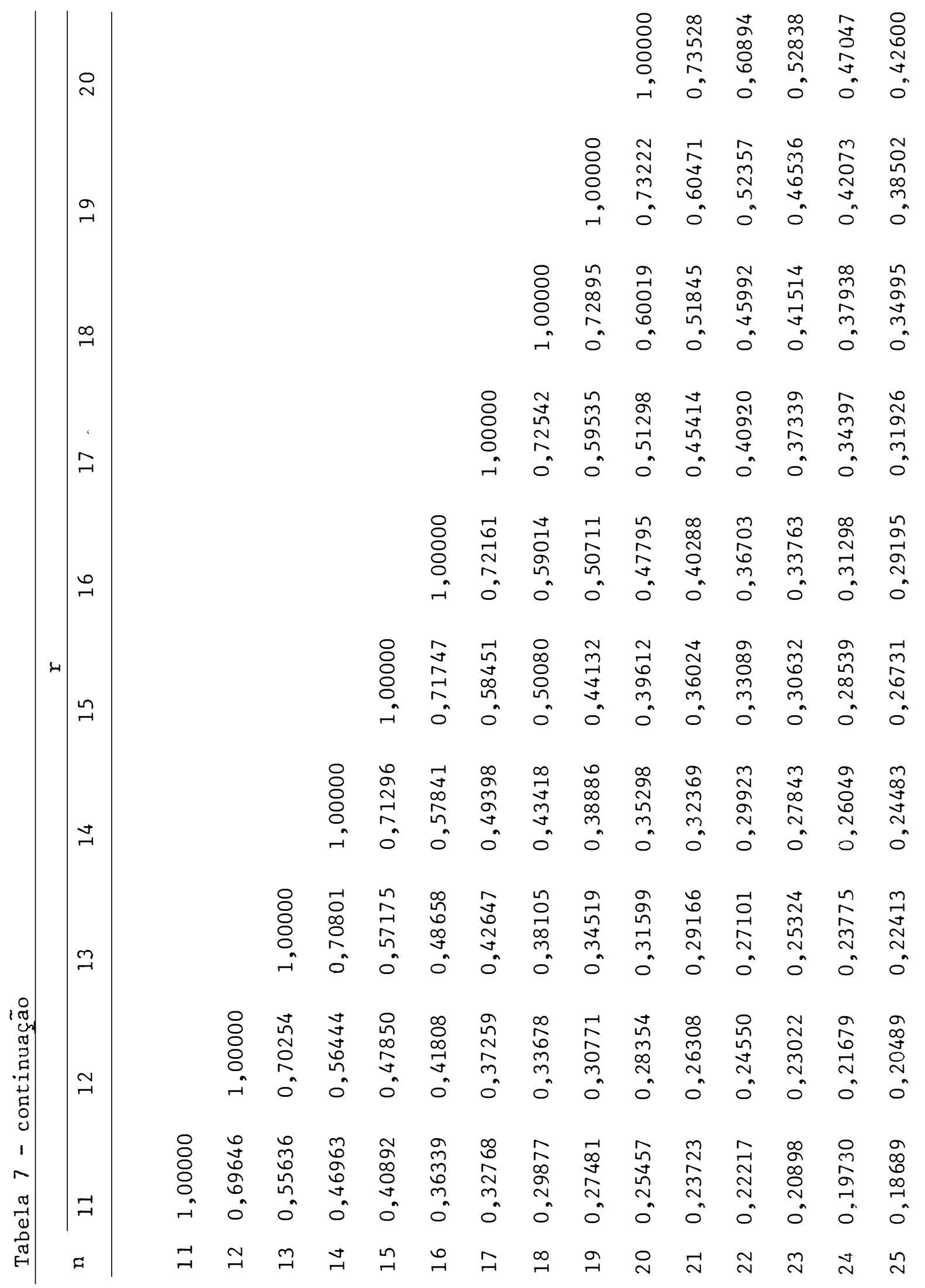




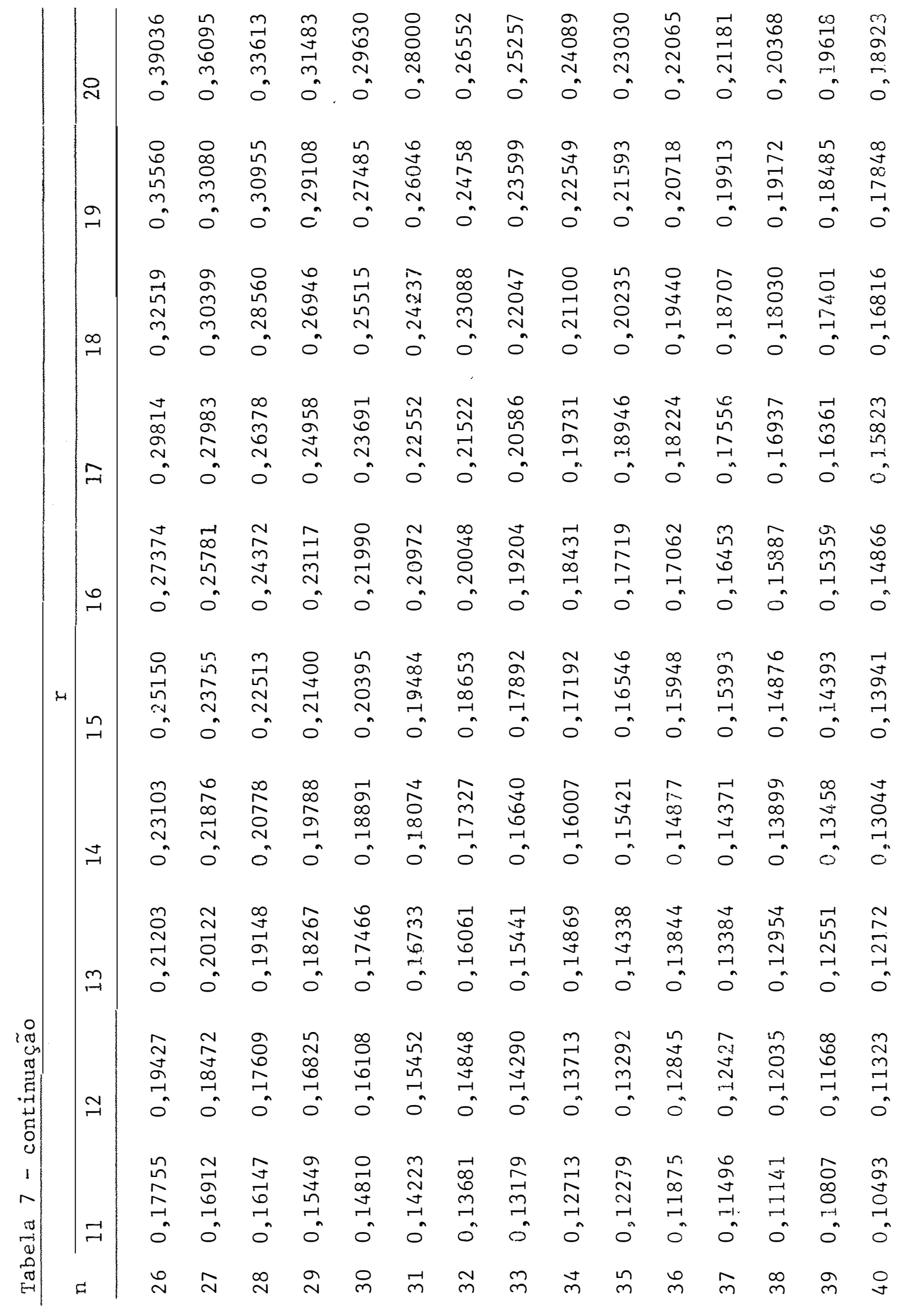




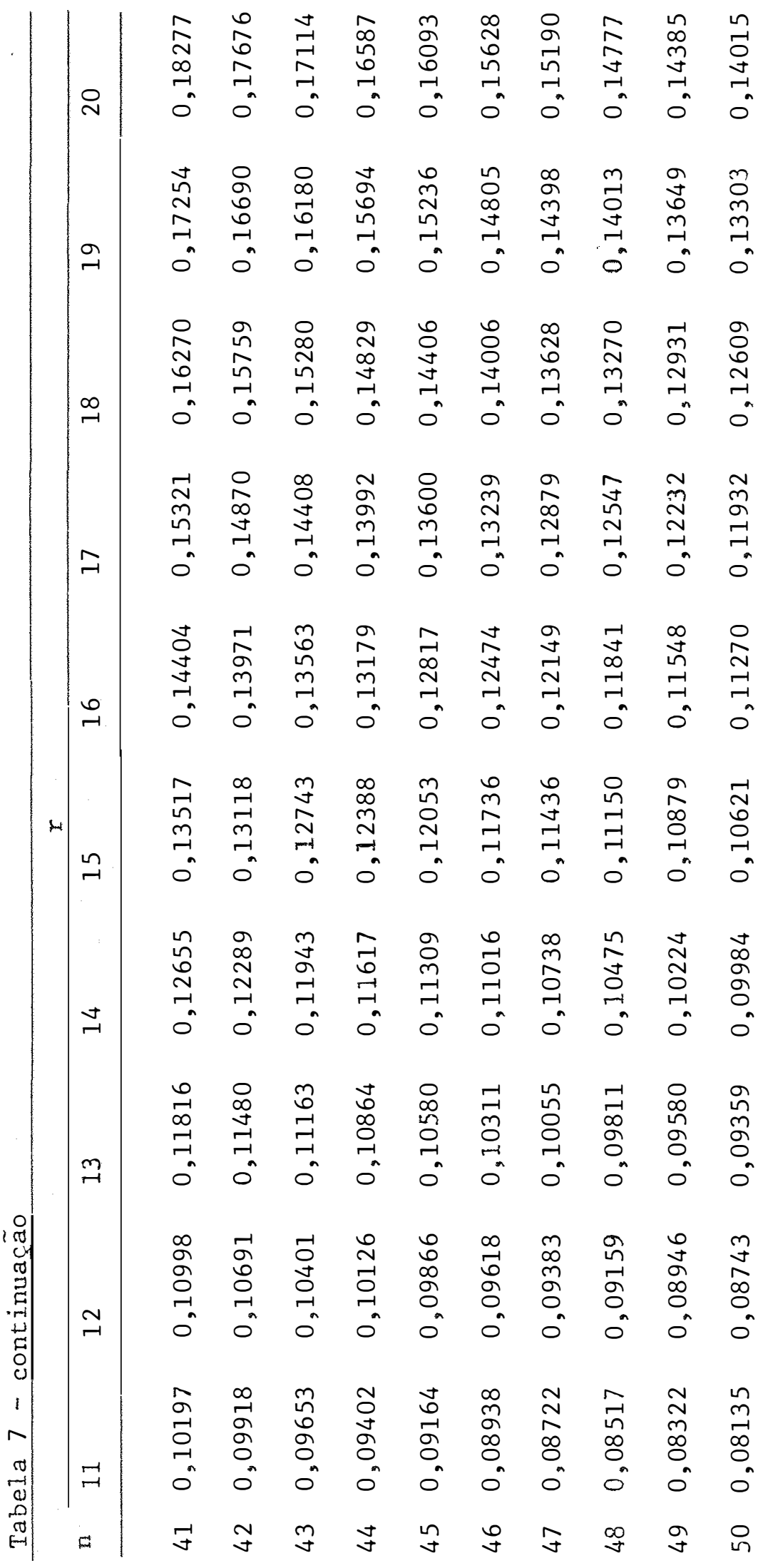




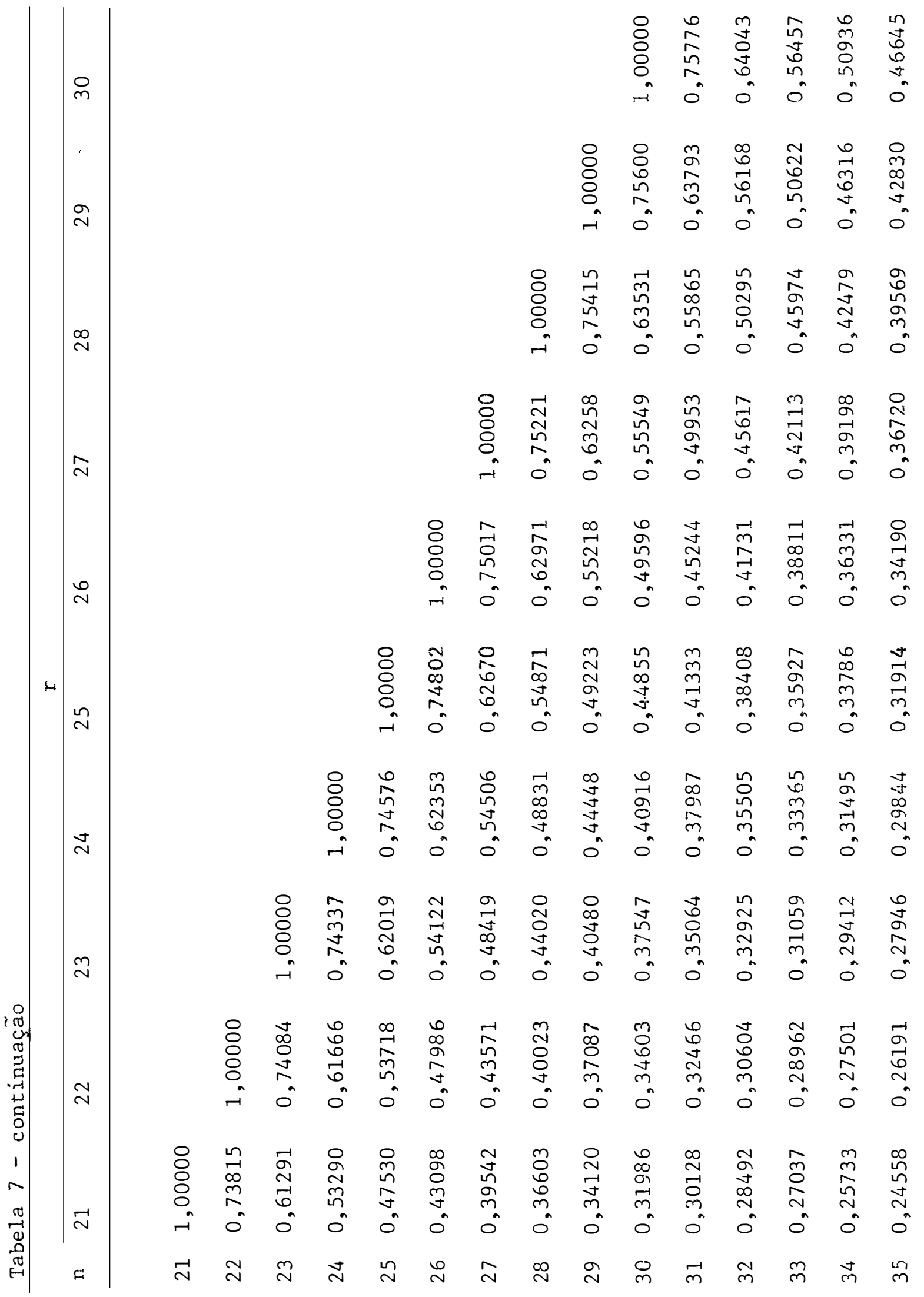




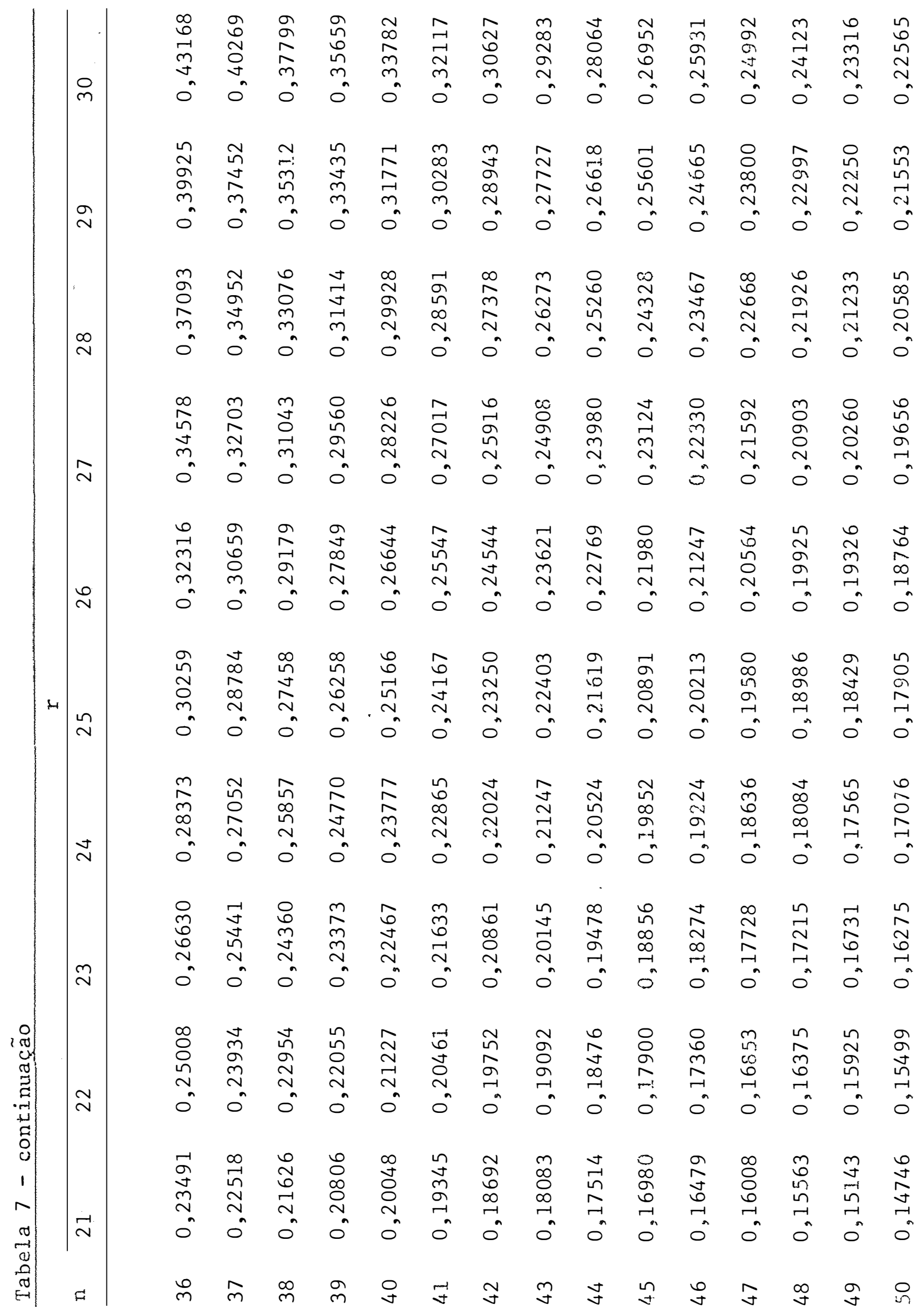




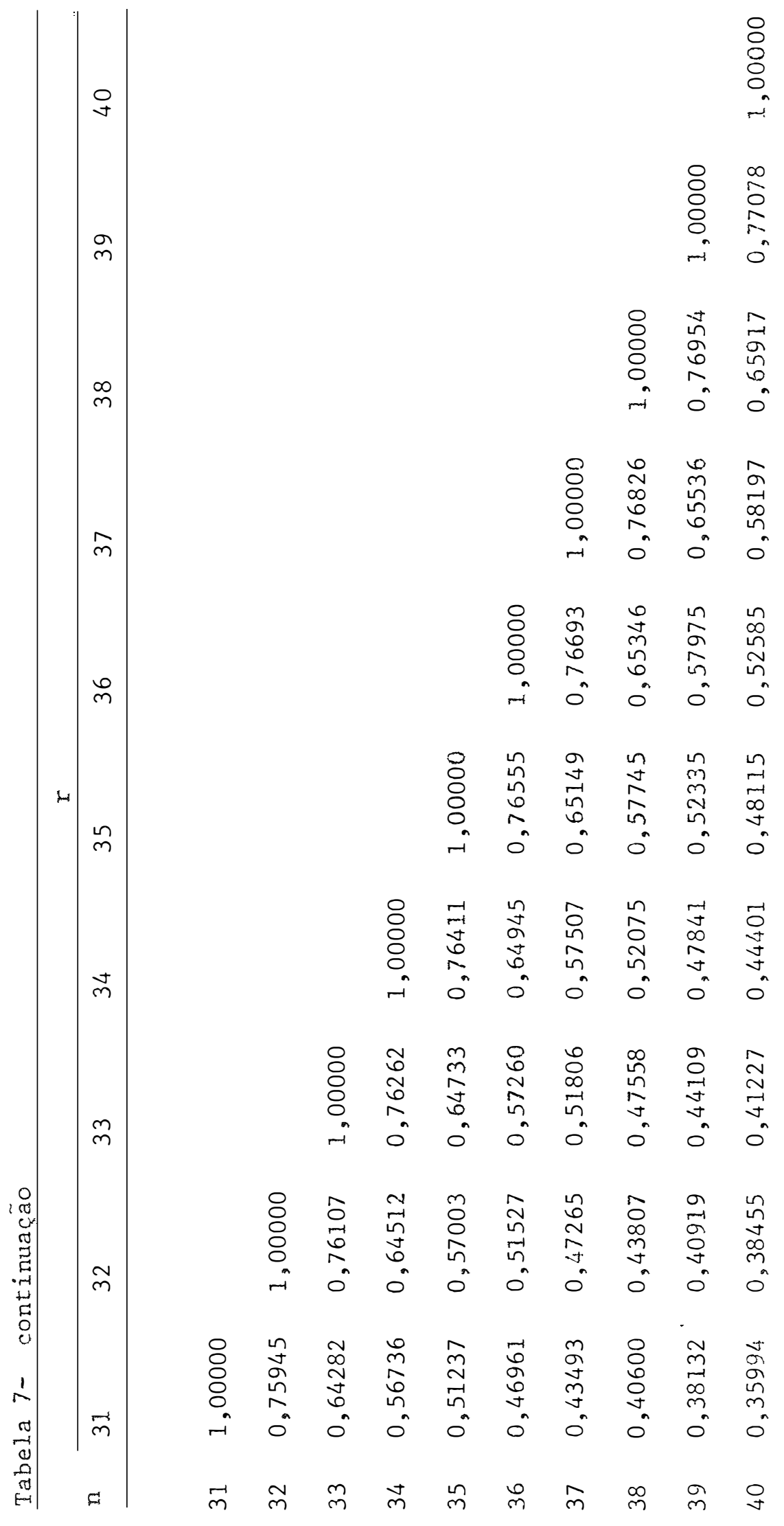




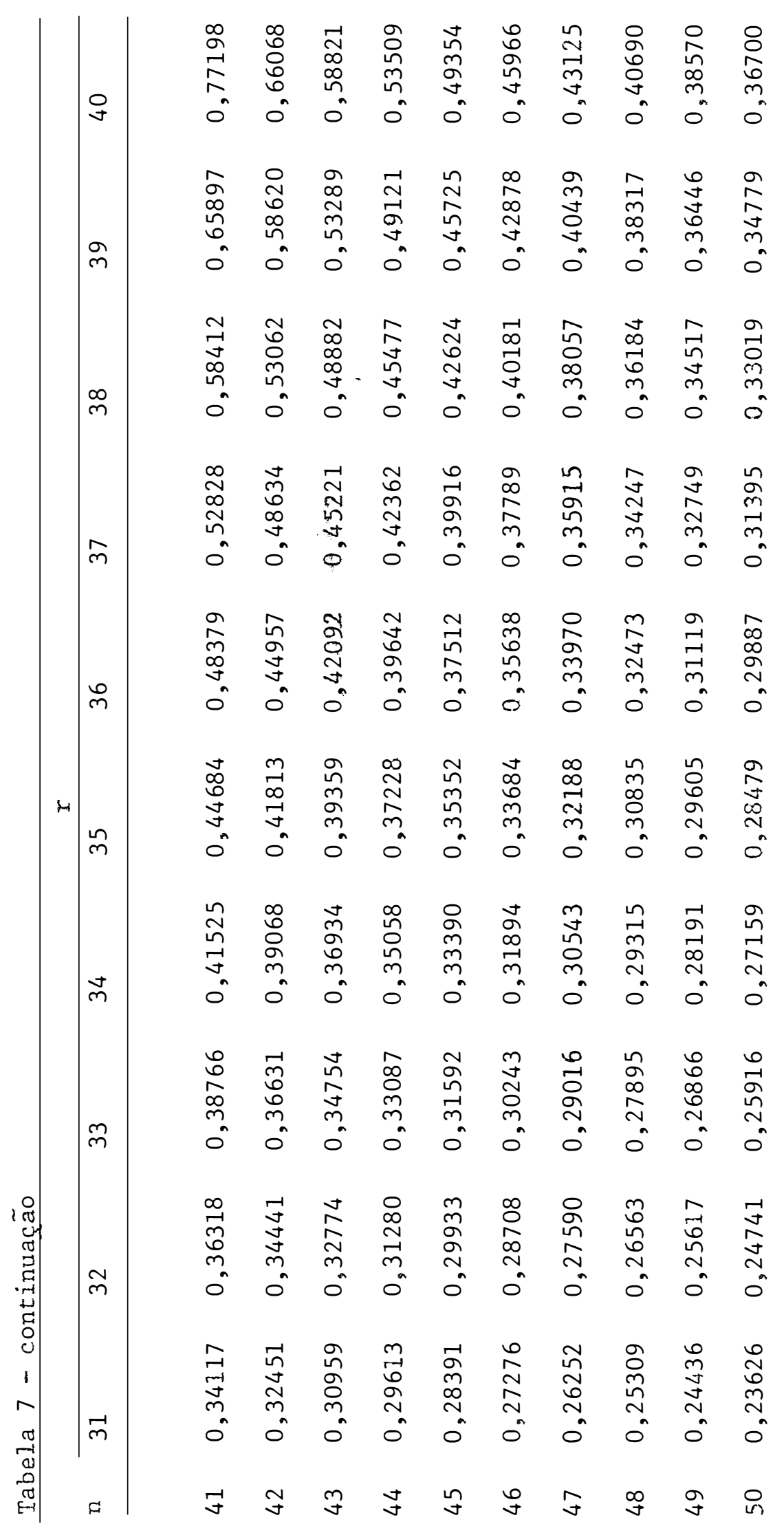


129.

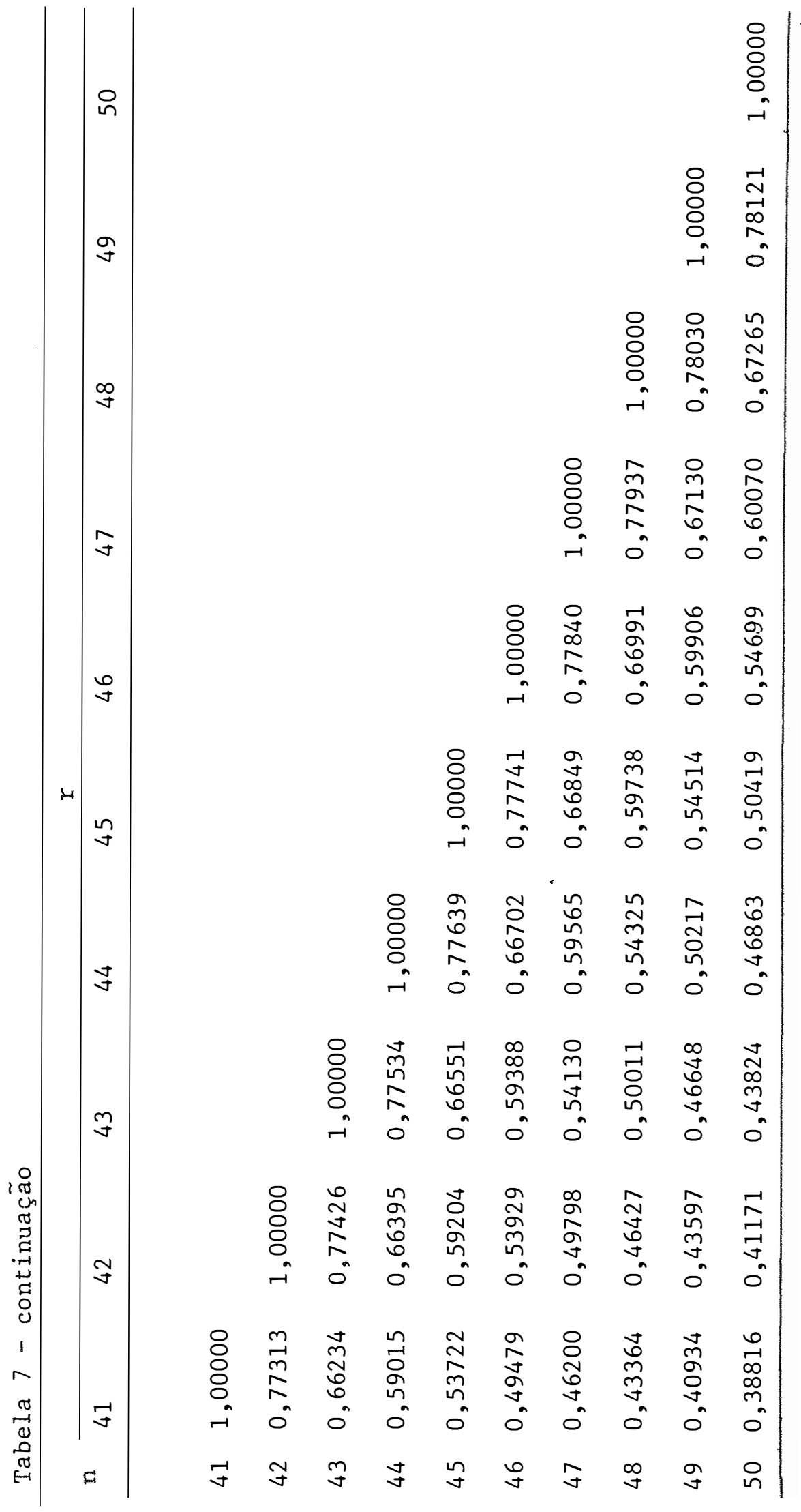


Tabela 8 - Estatîstica teste e regiões crîticas para os testes relativos a vida mêdia. Censura tipo I, amostragem sem reposição.

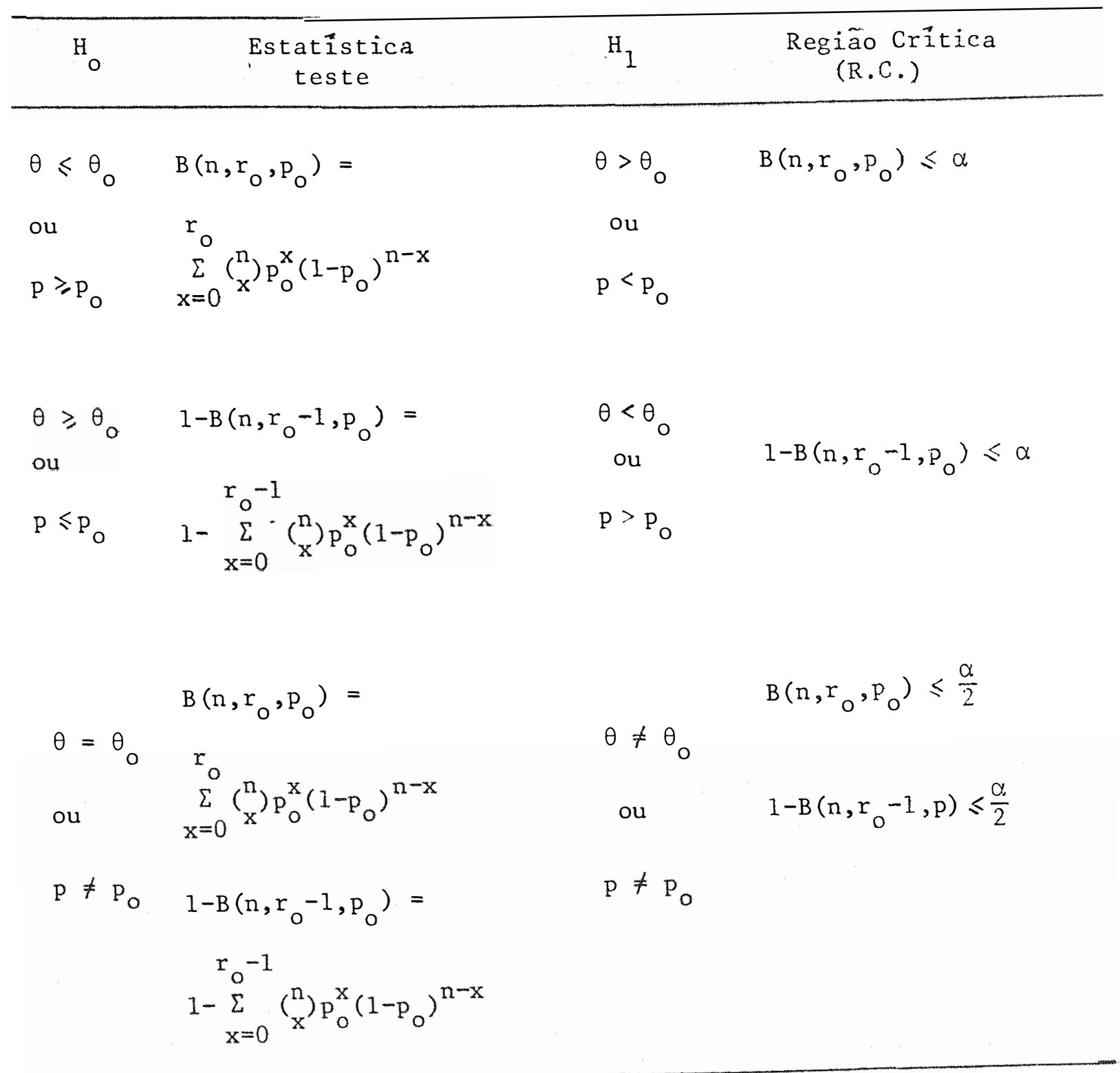


Tabela 9 - Estatistica teste e regióes criticas para os testes relativos a vida média. Censura tipo I, amostragem com reposição.

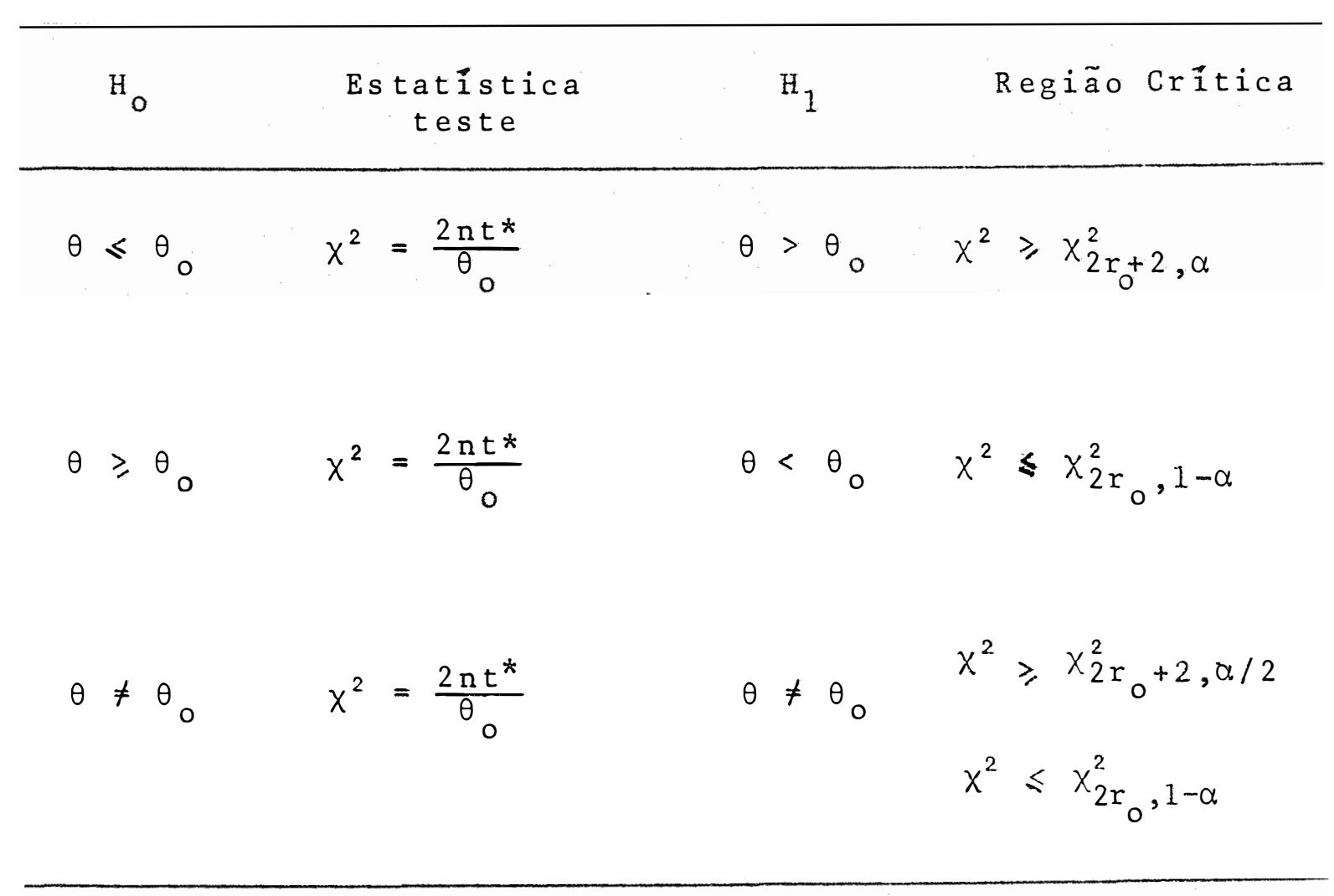

